\title{
DISPARITIES IN HEALTH
}

USE OF COMPLEMENTARY AND ALTERNATIVE MEDICAL THERAPIES IN CHINESE AND VIETNAMESE PATIENTS: PREVALENCE AND ASSOCIATED FACTORS. A. Ahn ${ }^{1}$, Q. Ngo-Metzger ${ }^{1}$, A. Legedza ${ }^{1}$, M.P. Massagli ${ }^{1}$, B. Clarridge ${ }^{2}$, J. Moorhead ${ }^{2}$, R.S. Phillips ${ }^{1}$; ${ }^{1}$ Harvard University, Boston, MA; ${ }^{2}$ University of Massachusetts, Boston, MA (Tracking ID \#74419)

BACKGROUND: Asian Americans are one of the fastest growing ethnic minorities in the United States. Many Asian Americans come from cultural backgrounds that encourage traditional Asian medical practices. Limited data exists on the utilization of such complementary and alternative medical (CAM) therapies in this population.

METHODS: We surveyed 4410 Chinese and Vietnamese patients who were seen within the past month at one of 11 conventional clinics located in major cities throughout the United States. The survey was presented in four languages (English, Cantonese, Mandarin, or Vietnamese) to ensure ease of survey completion. It contained 72 questions; most asked about experiences with care at their clinic. In addition, we asked questions regarding respondents' demographics (age, sex, marital status, and education level), comfort with English language, self-rated health status, and utilization of CAM therapies. Questions regarding CAM therapies addressed use of 9 specific CAM modalities and communication about CAM use with clinic medical staff. We used multivariable logistic regression to identify factors associated with any prior CAM use. RESULTS: Out of 4410 patients surveyed, 3258 (74\%) returned completed questionnaires. Use of CAM therapies was common in the surveyed population; $66.2 \%$ of respondents had "ever used" some form of CAM therapy. Only $7.6 \%$ of these patients who utilized CAM therapies discussed their use with clinic medical personnel. Use of specific modalities differed between ethnic groups. For both Cantonese and Mandarin Chinese, use of herbal medicine $(61.0 \%$ and $44.3 \%$, respectively) and acupuncture ( $21.4 \%$ and $20.9 \%$, respectively) was most common. For Vietnamese, the use of coining $(59.1 \%)$ and massage $(48.9 \%)$ was most common. In logistic multivariable analyses of factors associated with CAM use (after adjustment for site of care), individuals who spoke Vietnamese (AOR 2.3, 95\% CI $[1.8,3.0]$ ) or Cantonese $(2.2$, $[1.7,2.8])$ were more likely to use CAM therapies compared to Mandarin speaking individuals. Other factors associated with increased use were having 13 or more years of education (2.0, $[1.4,2.7])$ and perception of poor health $(1.4,[1.1,1.8])$. Gender, age over 65 , marital status, and poor rating of medical care (received at their clinic) were not associated with CAM use. CONCLUSION: Use of CAM therapies is common in Chinese and Vietnamese Americans. Despite this fact, discussion of their use with clinic medical staff was uncommon. Increased discussion of CAM use and further understanding of CAM therapies (and fundamental beliefs that underlie them) may help provide culturally sensitive health care for this population.

REGIONAL DIFFERENCES IN THE ASSOCIATION BETWEEN RACE AND HEALTH CARE SYSTEM DISTRUST. K. Armstrong ${ }^{1}$, A. Rose ${ }^{1}$, N. Peters ${ }^{1}$, J.A. Shea ${ }^{1}$; ${ }^{1}$ University of Pennsylvania, Philadelphia, PA (Tracking ID \#76469)

BACKGROUND: Little is currently known about how health care related distrust and racial differences in distrust may differ by region of the country.

METHODS: We conducted a random digit dialing survey of the adult continental US population to investigate the correlates of distrust of the health care system. Distrust was measured using a 10 item scale we developed (previously found to have a Cronbach's alpha of 0.75 and to load on a single factor accounting for $90 \%$ of the variance). Example items are: "Medical experiments can be done on me without my knowing about it"; "Medical records are kept private"; "When they take my blood, they do tests they don't tell me about"; "If a mistake were made in my health care, the health care system would try to hide it from me"; and "The health care system puts my medical needs above all other considerations when treating my medical problems" and "Some medicines have things in them that they don't tell you about". In this analysis, we restricted our sample to individuals who self-identified as either AfricanAmerican or Caucasian and lived in one of the two regions of the country that had a significant proportion of African-American respondents (the North-East and South). Multivariate regression was used to assess the associations between distrust and race and region as well as 
potential interactions. Distrust was primarily analyzed as a continuous variable. However, the resutls were similar if is dichotomized as high $(>=30)$ vs. low $(<30)$ distrust.

RESULTS: Of the 525 participants, $461(89 \%)$ were Caucasian and $57(11 \%)$ were AfricanAmerican. Mean age was 45 years; $52 \%$ were women; $59 \%$ had completed college; $86 \%$ had health insurance and $91 \%$ had a regular source of care. The mean distrust score was 30.7 with a range from 10 to 49. After adjustment for age, gender, education and income, distrust was associated with lack of insurance $(\mathrm{p}=0.03)$ and being 35 to 44 years old $(\mathrm{p}=0.02)$ but not with race or region. However, the interaction between race and region of the country was significant $(\mathrm{p}=0.02)$, with African-American race being significantly associated with higher distrust in the Northeast (OR 4.64, 95\% CI 1.2-15.9 for high vs low distrust) but not in the south (OR 0.59, 95\% CI $0.29-1.20)$.

CONCLUSION: Racial differences in distrust of the health care system vary by region of the country. African-Americans are more likely to distrust the health care system in the Northeast but not in the South. This finding has implications for understanding the causes and consequences of distrust of the health care system and for national studies of health care related trust and distrust.

\section{LEARNING FROM GEOGRAPHIC VARIATION IN RACIAL DISPARITIES IN PROSTATE CANCER TREATMENT. K. Armstrong ${ }^{1}$, K. Ravenell ${ }^{1}$, M. Putt ${ }^{1}{ }^{1}$ University of Pennsylvania, Philadelphia, PA (Tracking ID \#76881)}

BACKGROUND: Exploring the distribution and determinants of geographic variation in racial disparities in prostate cancer treatment may provide insight into causal pathways.

METHODS: We analyzed data on men with localized prostate cancer from Surveillance Epidemiology and End Results (SEER) from 1992 to 1998. SEER is a population based cancer registry that includes 11 sites and 205 counties in the US. SEER collects information on patient age, race, marital status and county of residence, tumor characteristics, treatment and survival. We limited the current analyses to men between the ages of 40 and 89 and counties ( $\mathrm{n}$ $=20$ ) where at least $5 \%$ of the cases were African-American. County level data on mean household income, proportion of residents who are Caucasian, proportion of residents who have completed college, and proportion of businesses owned by minorities were obtained from US Census data. We used hierarchical regression models to assess the association between individual level variables, county level variables and use of prostatectomy.

RESULTS: Of the 80,654 men included in the analysis, 66,142 (82\%) were Caucasian and 14,512 (18\%) were African-American. 36\% had undergone prostatectomy. County level median household income ranged from $\$ 37,266$ to $\$ 70,819$, \% Caucasian from $36 \%$ to $88 \%$, $\%$ college educated from $17 \%$ to $45 \%$, and $\%$ minority businesses from $4 \%$ to $37 \%$. The association between African-American race and use of prostatectomy varied across counties, (ranging from OR 0.43 to OR 1.32, LR test for interaction between race and county $\mathrm{p}=$ 0.0003). After adjustment for age, marital status, stage and grade, use of prostatectomy was associated with county level racial composition (OR 1.02 for each $1 \%$ increase in percent Caucasian, $\mathrm{p}<0.0005$ ), household income (OR 0.99 for each $\$ 1$ increase, $\mathrm{p}<0.0005$ ), and proportion of minority businesses (OR 1.03 for each $1 \%$ increase, $\mathrm{p}<0.0005)$ as well as patient race (OR 0.74 for African-American vs. Caucasian, $\mathrm{p}<0.001$ ). In addition, there was a significant interaction $(\mathrm{OR} 0.99, \mathrm{p}=0.02)$ between the effect of race and the proportion of minority businesses, with greater racial disparities in use of prostatectomy in counties with higher proportions of minority owned businesses.

CONCLUSION: Substantial geographic variation exists in the size of the racial disparity in treatment for localized prostate cancer. Counties with a higher proportion of businesses owned by minorities have greater racial disparities in use of prostatectomy. Our current research seeks to determine whether this association can be explained by the effect of residential racial segregation on the proportion of minority owned businesses and racial disparities in treatment.

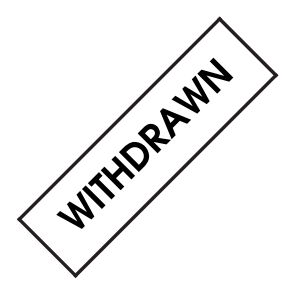

DIFFERENCES IN KNOWLEDGE, SELF-EFFICACY, EMPOWERMENT \& LITERACY AMONG DIABETIC PATIENTS. P.F. Bass ${ }^{1}$, M.A. Moore ${ }^{1}$, W. Rising ${ }^{1}$, C.S. Ritchie ${ }^{1}$; ${ }^{1}$ University of Louisville, Louisville, KY (Tracking ID \#76789)

BACKGROUND: Patients with poor literacy skills suffer a number of poor health outcomes. Factors contributing this, however, remain relatively unexplored. We hypothesized diabetic patients with poor literacy will have decreased knowledge, self efficacy in dealing with their physician, and less self-empowerment regarding diabetes.
METHODS: During a 8 week period in 2002 the General Internal Medicine Clinic (IMC) at the University of Louisville, we assessed diabetic patients' knowledge, self-efficacy, and empowerment. Health literacy was measured using the Rapid Estimate of Adult Literacy in Medicine- Revised (REALM-R), a new instrument to quickly screen for potential health literacy problems. Patient's self-efficacy in obtaining medical information and having physicians address their concerns was measured using the Perceived Efficacy in PatientPhysician Interactions (PEPPI) questionnaire. Knowledge of diabetes was assesses using the Michigan Diabetes Research Training Center Brief Diabetes Knowledge Test. The Diabetes Empowerment Scale (DES) measures psychosocial issues, readiness to change, and ability to set diabetic treatment goals for. All questions were read to patients and patients were encouraged to ask questions if they did not understand.

RESULTS: A convenience sample of 104 consented to participate in the study with $51 \%$ being female and an average age of 52 . Ninety percent were type II diabetics with $64 \%$ on oral medication and $49 \%$ on insulin. Based on REALM-R scores, $68 \%$ of participants were at risk for poor literacy. Patients with a positive screen (scoring 6 or less on the 8 item REALM-R) scored lower on the knowledge test ( 47 vs. $64 \mathrm{p}<.000$ ). Scores on the DES (3.6 vs $3.7 \mathrm{p}=.183$ ) and PEPPI (Kruskal Wallis test for median differences $\mathrm{p}=.947$ ) were not different between the positive and negative screened groups.

CONCLUSION: Diabetic patients at risk for poor literacy displayed poorer knowledge about their chronic disease. While self-efficacy has been shown to mediate health outcomes positively, self-efficacy among patients positively screening for poor health literacy did not differ from those with a negative screen. Similarly, positive screening patients were no less empowered than negatively screened patients. Thus by self-report, patients screening positively appear to interact equally well with physicians compared to those with negative screens and are no less empowered regarding their diabetes. If this were substantiated in subsequent studies, it would suggest that self-efficacy and empowerment should not be targets of intervention to improve health outcomes between literate and poorly literate patients.

IMPROVING FOLLOW UP CARE TO MINORITY WOMEN WITH BREAST ABNORMALITIES. T.A. Battaglia ${ }^{1}$, K. Kim ${ }^{1}$, M. Posner ${ }^{1}$, C. Chapman ${ }^{1}$, J. Han ${ }^{2}$, K.M. Freund ${ }^{1}$; ${ }^{1}$ Boston University, Boston, MA; ${ }^{2}$ Rush Presbyterian St. Luke's Medical Center, Chicago, IL (Tracking ID \#74562)

BACKGROUND: Delays in follow up for abnormal breast cancer screening impact survival. We previously found significant delays in follow up after abnormal breast cancer screening in minority, low income women. This study aimed to: 1) evaluate a case management intervention to improve follow up and 2) identify factors associated with inadequate follow up.

METHODS: Communication and coordination of services were identified as major barriers to keeping a scheduled appointment for evaluation of a breast abnormality. Therefore, a full time case coordinator was charged with contacting all scheduled patients, reviewing plans for her appointment, assisting with logistics such as transportation or obtaining prior mammograms, and facilitating communication among providers. The study population included consecutive, unique patients referred to a Breast Center at an urban medical center from March to August 2001 and March to August 2002. Adequate follow up was defined as patient arrival to a scheduled appointment. Demographic information was collected via computerized registration files, clinical information was collected via patient interview and the medical chart.

RESULTS: Of the 790 patients referred to the Breast Center during the study period, $50 \%$ were between ages 40-64, 35\% were African American, 36\% Caucasian, and 16\% Hispanic. $58 \%$ had public or no health insurance. $48 \%$ were referred from neighborhood health centers, $40 \%$ from hospital based practice sites. During the study intervention, $77 \%$ had adequate follow up compared with $64 \%$ prior to the intervention $(\mathrm{p}<0.0001)$. Predictors of inadequate follow up included: 1) Age, $52 \%$ of women aged 18-39 had inadequate follow up compared with $42 \%$ ages $40-64$, and $6 \% 65$ and older ( $<0.0001) ; 2$ ) Race, $43 \%$ of African Americans had inadequate follow up compared with $25 \%$ of Caucasians $(\mathrm{p}<0.001) ; 3)$ Insurance Status, $69 \%$ of women with no health insurance or public health insurance had inadequate follow up compared with $31 \%$ of women with private health insurance (p,0.002); and 4) Referral source, $56 \%$ of women referred from a Neighborhood Health Center had inadequate follow up compared with $38 \%$ referred from a hospital based practice $(\mathrm{p}<0.002)$.

CONCLUSION: In a cohort of predominantly low-income minority women, use of a case coordinator significantly improved rates of follow up in women referred for evaluation of a breast abnormality. Intensive case coordination, not routinely covered by health insurance, is necessary to ensure adequate follow up of at risk populations.

PHYSICIAN PERSPECTIVES ON TRUST, PARTNERSHIP, AND COMMUNICATION WITH PATIENTS: DOES ETHNICITY MATTER? M.C. Beach ${ }^{1}$, L.E. Boulware ${ }^{1}$, D.L. Roter ${ }^{1}$, L.A. Cooper ${ }^{1}$; ${ }^{1}$ Johns Hopkins University, Baltimore, MD (Tracking ID \#76954)

BACKGROUND: Previous studies examining the relation between ethnicity and patientphysician trust, partnership, and communication have focused on patients' perspectives. This study examines whether patient and physician ethnicity are related to physician-reported measures of trust, partnership and communication with patients.

METHODS: We analyzed a survey of 3113 physicians practicing in the state of Maryland commissioned to study physician experience with managed care. Physicians were asked to rate their level of ability to communicate effectively with patients, engender patients' trust, and establish patients as partners. Physicians were also asked about their own demographic information, their experiences with managed care, and the ethnicity of their patient populations. Using multivariable logistic regression, we evaluated the relation of both physician and patient population ethnicity to physician ratings of trust, partnership, and communication with patients. RESULTS: Of 1650 physicians responding (response rate $53 \%$ ), 64\% were ethnic minorities (28\% Asian/Pacific Islander, 24\% African-American, and 12\% Hispanic), and 34\% were White. One-half $(48 \%)$ of physicians reported that $>50 \%$ of their patients were ethnic minorities. After adjusting for potential confounders, ethnic minority physicians were less likely than their White counterparts to report being able to communicate effectively with patients, regardless of their patient population ethnicity (O.R. 0.59, $95 \%$ C.I $0.35-0.99)$. Physicians with 
$>50 \%$ minority patients were less likely than their counterparts to report being able to engender patients' trust (O.R. 0.73, 95\% C.I. $0.55-0.97$ ) and less likely to report being able to establish patients as partners (O.R. 0.63, 95\% C.I. 0.47-0.86) regardless of physician ethnicity. There were no significant interactions between physician and patient population ethnicity. CONCLUSION: While physician-reported ability to communicate effectively with patients is related to physicians' ethnicity, physician-reported abilities to establish trust and partnership with patients are related to the ethnicity of physicians' patient population. Future research on the influence of ethnicity in patient-physician relationships should include both patients' and physicians' perspectives and should seek to identify the mechanisms for differential interpersonal dynamics.

DISPARITIES IN HEALTH LITERACY BETWEEN AFRICAN AMERICAN AND NONAFRICAN AMERICAN PRIMARY CARE PATIENTS. B.B. Beers ${ }^{1}$, V.J. McDonald ${ }^{1}$, D.A. Quistberg ${ }^{1}$, K.L. Ravenell ${ }^{1}$, D.A. Asch ${ }^{1}$, J.A. Shea ${ }^{1}$; ${ }^{1}$ Center for Health Equity Research and Promotion-PVAMC, Philadelphia, PA (Tracking ID \#76502)

BACKGROUND: Health literacy has been linked to many health seeking behaviors and health outcomes. Rigorous studies of health literacy require a psychometrically sound assessment tool. One of the most widely used instruments is the Rapid Estimate of Adult Health Literacy (REALM). Previous studies have focused on overall REALM scores, their relationship to knowledge and attitudes, with some exploration of differences across demographic subgroups. Our objectives were to examine 1) REALM scores in a large and diverse sample of primary care patients and 2) disparities in total and item scores related to education, race, gender and age. METHODS: Patients were recruited in primary care clinic waiting areas at a VA medical center and a university health system. Patients reported sociodemographic information and were administered the REALM, a word recognition test that evaluates patient pronunciation of 66 common medical terms. Items are scored 1 if the item is pronounced correctly and 0 if it is mispronounced. ANOVAs were used to compare total and item scores across analytic groups of interest: education, race, sex, and age.

RESULTS: REALM was administered to 1805 patients. The sample was $58 \%$ African American; $66 \%$ were men; education levels were $14 \%$ less than high school, $36 \%$ high school, $31 \%$ some college, $20 \%$ college plus. The mean REALM score was 58 (range 0 to 66 ). Cronbach's alpha was .96. REALM scores were significantly related to education $(\mathrm{p}<.0001)$, and they were also significantly lower for African Americans $(\mathrm{p}<.0001)$, men $(\mathrm{p}=.003)$, and older patients $(\mathrm{p}=.0001)$. Differences by age and sex dissipated when stratifying by level of education, whereas racial differences persisted at the three lowest education levels. African Americans had lower total REALM scores than non-African Americans $(\mathrm{p}<.0001$ for all comparisons). Item analyses within education levels showed that African Americans had significantly lower scores on many of the 66 items: 19 in less than high school, 48 in high school, and 23 in some college groups.

CONCLUSION: As a whole, the REALM is an extremely robust assessment of health literacy. While differences in scores related to education were expected, we found persistent and unexplained differences associated with race. Item level review added to our understanding of the potential biases in the overall instrument. A health literacy scale should behave similarly when applied to populations at equivalent levels of education. The discordance between African Americans and Caucasians in REALM scores needs to be further addressed as it may reflect problems with the instrument, or more general issues surrounding assessment of education.

IS THERE A DIGITAL DIVIDE AMONG PHYSICIANS? A GEOGRAPHIC ANALYSIS OF INFORMATION TECHNOLOGY IN PHYSICIAN OFFICES. D.S. Bell ${ }^{1}$, D.M. Daly ${ }^{2}$ P.L. Robinson ${ }^{3} ;{ }^{1}$ University of California, Los Angeles, Los Angeles, CA; ${ }^{2}$ CalOptima Health System, Orange, CA; ${ }^{3}$ California State University at Northridge and Charles R. Drew University, Los Angeles, CA (Tracking ID \#77025)

BACKGROUND: Surveys of the U.S. population have identified a "Digital Divide," with African Americans and Hispanics having less access to the Internet than majority groups do. As more health-related services move to the Internet, the Digital Divide could contribute to a worsening of health disparities. The objective of this study was to determine if differential access existed for physician's offices operating in minority and poor communities compared with those located in non-minority and wealthier areas.

METHODS: CalOptima, the county-organized health system of Orange County, California, surveyed contracting pediatrician, family medicine, and general practice offices to assess their readiness for and interest in online services. We located each surveyed office in a 2000 Census tract using a geographical information system. We categorized Orange County Census tracts based on the proportions of residents having Hispanic ethnicity, non-white race, and povertylevel incomes. Category boundaries were set to minimize within-category variance using a Jenks optimization algorithm. Cochran-Armitage tests were used to assess trends in office characteristics across these ordinal categories.

RESULTS: Of 307 offices surveyed, 141 responded (46\%). Response rates were not associated with office location. Of the responding offices, $94 \%$ had a computer, $77 \%$ had Web access, $29 \%$ had broadband Web access (cable modem, DSL, or T1), and 53\% used a computerized practice management system. Offices located in poor and minority communities had equivalent use of each technology. For example, among the 33 responding offices in the highest-Hispanic areas (68-98\% Hispanic), all had a computer, $85 \%$ had Web access, and $27 \%$ had broadband access. Among the 16 offices in the highest-poverty areas (24-68\% in poverty), all had a computer, $88 \%$ had Web access, and $31 \%$ had broadband. Offices reported high interest in online patient tracking, but also high levels of concern about its usability and confidentiality. There were no differences in these attitudes by office location, but offices with Web access and those with practice management systems expressed both greater interest and also greater concerns.

CONCLUSION: Primary care physicians practicing in poor and minority communities in a large, suburban county had equitable access to and interest in Web-based systems. These results suggest that generalists may be productive targets for online services intended to reduce health disparities. Additional research is required in more traditional urban settings to determine whether this conclusion is widely applicable.
THE RELATIONSHIP BETWEEN NEIGHBORHOOD POVERTY AND HEALTH STATUS FOR PERSONS WITH CHRONIC CONDITIONS. A.F. Brown ${ }^{1}$, A. Ang ${ }^{1}$, A.R. Pebley'; ${ }^{1}$ University of California, Los Angeles, Los Angeles, CA (Tracking ID \#76635)

BACKGROUND: Health outcomes have been linked to neighborhood context even when individual attributes are taken into account, but the relationship between neighborhood characteristics and health for persons with a chronic condition is poorly understood. METHODS: The Los Angeles Family and Neighborhood Study (LA FANS) is a longitudinal study based on a representative sample of families in 65 neighborhoods in Los Angeles County, with an oversample in poor neighborhoods. Census data were used to classify neighborhoods as very poor (top $10 \%$ of the poverty distribution), poor (60-89th percentiles of poverty), and nonpoor (bottom 60 percent of poverty distribution). Information on individual characteristics was obtained from in-person surveys. Ordered logistic regression was used to evaluate the relationship between neighborhood poverty and self-reported health, ranked from 1 (Poor) to 5 (Excellent), after adjustment for individual characteristics.

RESULTS: Among the 2623 ( $70 \%$ response rate) adult respondents included in this analysis, mean age 40 years, $36 \%$ reported one or more chronic conditions. Health status was reported as excellent by $21 \%$ of respondents, very good $25 \%$, good $31 \%$, fair $19 \%$, and poor $4 \%$. In multivariate models, poorer health status was associated with the presence of a chronic condition ( $p<0.001$ ), less education ( $p=0.002$ ), and living in a very poor or poor neighborhood $(p=0.04)$. The gradient in health status between persons with and without a chronic condition was significantly steeper in poor and very poor than in non-poor neighborhoods. (See table.) CONCLUSION: Residing in a less affluent neighborhood is associated with worse health status for adults with a chronic condition, but not those without a chronic disease. Further study is needed to identify reasons for these area-level disparities in health status among persons with chronic conditions.

Adjusted Odds Ratios for Health Status by Chronic Illness and Neighborhood Poverty

\begin{tabular}{lllc} 
& \multicolumn{1}{c}{ Non-Poor } & \multicolumn{1}{c}{ Poor } & Very Poor \\
No Chronic Illness & $1.00($ Reference $)$ & $0.98(\mathrm{p}=0.02)$ & $0.98(\mathrm{p}=0.01)$ \\
Any Chronic Illness & $0.79(\mathrm{p}=0.01)$ & $0.70(\mathrm{p}=0.01)$ & $0.66(\mathrm{p}=0.01)$ \\
Hypertension & $0.82(\mathrm{p}<0.001)$ & $0.68(\mathrm{p}<0.001)$ & $0.57(\mathrm{p}<0.001)$ \\
Arthritis & $0.66(\mathrm{p}<0.001)$ & $0.55(\mathrm{p}<0.001)$ & $0.46(\mathrm{p}<0.001)$ \\
Diabetes & $0.65(\mathrm{p}<0.001)$ & $0.54(\mathrm{p}<0.001)$ & $0.45(\mathrm{p}<0.001)$
\end{tabular}

THE EFFECT OF LITERACY AND ANTICOAGULATION KNOWLEDGE ON THE ADEQUACY OF WARFARIN ANTICOAGULATION FOR PATIENTS WITH ATRIAL FIBRILLATION. B. Bryant ${ }^{1}$, R. Malone ${ }^{1}$, D. Ayscue ${ }^{1}$, D.A. DeWalt ${ }^{1}$, M. Pignone ${ }^{1}$; ${ }^{1}$ University of North Carolina at Chapel Hill, Chapel Hill, NC (Tracking ID \#76548)

BACKGROUND: Warfarin therapy reduces the risk of ischemic stroke for patients with atrial fibrillation, but appropriate self-care, including adherence to medication and dietary recommendations, is required because of its narrow therapeutic index. Inadequate self-care could lead to suboptimal anticoagulation and may increase the risk for adverse outcomes. We sought to determine if low literacy or lack of adequate anticoagulation-related knowledge could lead to increased frequency of non-therapeutic international normalized ratio (INR) for patients followed in our general internal medicine-based anticoagulation service.

METHODS: All English-speaking adult patients taking warfarin for atrial fibrillation who were seen at least once in the UNC General Internal Medicine Anticoagulation Clinic between 6/1/2002 and 10/1/02 were eligible for enrollment. Literacy was measured using the Rapid Estimate of Adult Literacy in Medicine (REALM). To measure anticoagulation-related knowledge, we designed a novel, verbally administered 17 item questionnaire that was administered at enrollment. Our main clinical outcome was the fraction of INRs measured that were in the therapeutic range while on a steady-state warfarin dose. Steady state dose was defined as 10 days of continuous warfarin therapy. All INRs measured for one year were included in the analysis. Descriptive statistics, analysis of variance and linear regression were used to assess the relationships between the hypothesized predictor variables and the outcome. RESULTS: 71 eligible patients with atrial fibrillation made at least one visit to the Anticoagulation Clinic from 6/01/2002 to $10 / 01 / 2002$. 8 refused and 5 were not assessed. Fifty-eight patients participated. Mean age was 73 (range $51-89$ ); $53 \%$ were men; $27 \%$ were African-American; $84 \%$ were married; and $64 \%$ had an income under $\$ 20,000$ per year. On the REALM, $21 \%$ scored $<=3$ rd grade, $14 \% 4-6$ th grade, $14 \% 7-8$ th grade and $52 \%>=9$ th grade. Knowledge scores increased with higher literacy levels. (mean number of correct answers 12.2, $12.5,13,15, p=0.004$ for trend). Sixty-nine percent of INR values were within therapeutic range. Proportion of therapeutic INR values did not vary according to literacy level or knowledge score. The proportion of patients with at least one INR $>4.0$ did not differ according to literacy level. CONCLUSION: Consistent with previous studies, patients with low literacy score lower on knowledge assessment. However, neither literacy nor knowledge predicted better control of anticoagulation. These findings suggest that a pharmacist managed anticoagulation program that is sensitive to the needs of low-literacy populations can prevent disparities in successful use of anticoagulation for atrial fibrillation.

ASSESSING THE QUALITY OF LIFE OF AFRICAN-AMERICAN AND CAUCASIAN MEN WITH PROSTATE CANCER. $\underline{\text { H. Burke }}{ }^{1}$, J. Cooper ${ }^{1}$; ${ }^{1}$ George Washington University, Washington, DC (Tracking ID \#76792)

BACKGROUND: Improving the quality of life of men undergoing active therapy for prostate cancer requires an instrument that can accurately assess their physical and mental health. In addition, we would like to determine if there are significant quality of life differences between African-American and Caucasian men with prostate cancer.

METHODS: The study population consisted of 161,250 patients who responded to a Medicare 1998 survey. There were 54,685 men of whom 2,985 were undergoing active therapy for prostate cancer. The first analysis was to determine if the eight SF-36 subscales could distinguish between men undergoing active treatment for prostate cancer vs. all other men. The second analysis was to determine if the SF-36 could distinguish men undergoing prostate cancer therapy by race, African-American vs. Caucasian. Both analyses are adjusted for age, martial status, and 
education. Multivariate logistic regression modeled each subscale-outcome relationship, we report the area under the receiver operating characteristic curve (ROC) of the model. RESULTS: For each SF-36 scale the ROCs of men with vs. without prostate cancer were: bodily pain, 0.617 , physical function, 0.613 , social function, 0.619 , general health, 0.633 , vitality, 0.620 , role physical, 0.626 , role emotional, 0.611 , mental health, 0.608 . For each SF-36 scale the ROCs for AA men vs. Caucasian men with prostate cancer were: bodily pain, 0.661 , physical function, 0.660 , social function, 0.663 , general health, 0.668 , vitality, 0.669 , role physical, 0.661, role emotional, o.664, and mental health, 0.662. All the scales were significantly $(\mathrm{P}<0.05)$ better than chance at distinguishing the men with prostate cancer from those without prostate cancer and all the scales were significantly $(\mathrm{P}<0.05)$ more accurate at distinguishing between African-American and Caucasian men with prostate cancer. CONCLUSION: Across the SF-36 subscales the men with prostate cancer were significantly different from those with without prostate cancer. Across the SF-36 subscales African-American men with prostate cancer were significantly different from Caucasian with prostate cancer.

DOES HEALTH INSURANCE MATTER FOR IMMIGRANTS. $\underline{\text { O. Carrasquillo }}{ }^{1}, \mathrm{~S}$. Pati $^{2}$, ${ }^{1}$ Columbia University, New York, NY; ${ }^{2}$ University of Pennsylvania, Philadelphia, PA (Tracking ID \#75926)

BACKGROUND: Half of all recent immigrants are uninsured. In this study, we examine the role of health insurance and having a usual source of care (USC) on utilization of cancer screening services among immigrant women.

METHODS: We analyzed data from the Commonwealth Fund Survey on Disparities in Quality of Health Care. This nationally representative telephone survey collected information on the health care experiences of patients of various racial and ethnic backgrounds. Persons born outside the United States (US) were classified as immigrants. Those who had resided in the US for under 10 years were recent immigrants; LT immigrants are those with a longer tenure. Based on conceptual models of access to cancer services, logistic regression was used to adjust for differences in socio-demographics, health attitudes/ beliefs, patient/provider communication and the medical care environment.

RESULTS: The HCOS sampled 3,622 women aged 18-70. The 822 women born outside the United States were representative of 12.1 million immigrant women. $48 \%$ (standard error [SE] $3 \%$ ) of recent immigrant women were uninsured versus $22 \%$ (SE $2 \%$ ) of LT immigrant and $14 \%$ (SE 1\%) of US born women. Among the uninsured, recent immigrants were more likely to lack a USC, while LT immigrants were more likely to receive care in a clinic and US born women most likely to receive care from a private doctor. $27 \%$ (standard error [SE] $4 \%$ ) of recent immigrant women aged 18-65 did not have a Pap smear and 28\% (SE 4\%) did not have a mammogram in the previous two years. $17 \%$ and $11 \%$ (SE 3\%) of LT immigrants and $11 \%$ and $11 \%$ (SE $1 \%$ ) of US born women, did not have these screenings, respectively. Among those with insurance or a USC, differences in screening between recent immigrants versus US born women were four percentage points or less. In multivariate models, insurance was the single strongest predictor of screening. However, recent immigrants remained several percentage points less likely to have such screening than US born women, but differences were no longer statistically significant; $73 \%$ versus $80 \%$ for pap smears $78 \%$ vs. $88 \%$ for mammography OR 0.66 CI $0.34-1.26$ and OR $0.45,0.08-2.54$, respectively. Use of interaction terms including lack of insurance among recent immigrants did not improve model fit.

CONCLUSION: Disparities in screening were greatly attenuated among the insured population. This finding supports efforts to extend coverage to recent immigrants. Among the uninsured, LT immigrants and US born women were more likely than recent immigrants to have a USC and receive screenings. Outreach to recent immigrants aimed at increasing awareness of available sources of care for the uninsured may also lessen disparities.

MORTALITY AMONG HOMELESS WOMEN. A.M. Cheung ${ }^{1}$, S.W. Hwang ${ }^{2}$; ${ }^{1}$ University Health Network, Toronto, Ontario; ${ }^{2}$ St. Michael's Hospital, Toronto, Ontario (Tracking ID \#76523)

BACKGROUND: About one-third of homeless people in the U.S. are women. While women in the general population have lower mortality rates than men, it is unknown whether this pattern persists among homeless people. The objectives of this study were to determine mortality rates among women using homeless shelters in Toronto, Ontario, and to analyze patterns of mortality among homeless women using all available pertinent data.

METHODS: We compiled a database of all single women and men aged 18 to 64 years who used a homeless shelter in Toronto in 1995. Deaths were ascertained using Ontario death certificate records for 1995-97. We performed a Medline search and manual review of references to identify all published studies providing data on mortality rates in cohorts of homeless women and men. The four studies identified were conducted in Boston (1988-93), New York City (198794), Philadelphia (1985-88), and Brighton, England (1981-92). Age-specific mortality rates were calculated for homeless women and men in Toronto and in the four cities noted above. In each city, rate ratios were calculated comparing mortality rates for homeless women vs. women in the general population and for homeless women vs. homeless men.

RESULTS: Deaths were identified among 1,981 women who used homeless shelters in Toronto in 1995 . Mortality rates were 515 and 438 (deaths per 100,000 person-years of observation) among women age 18-44 and 45-64, respectively. Mean age at death was 39 years. The most common causes of death in women age 18-44 were HIV/AIDS and drug overdose. Across all 5 cities, mortality rate ratios for homeless women vs. women in the general population were 4.610.1 in the younger age group and $1.0-1.5$ in the older age group. Mortality rate ratios for homeless women vs. homeless men were $0.4-1.1$ in the younger age group (significantly less than 1.0 in Boston only). Mortality rate ratios for homeless women vs. homeless men were $0.3-0.5$ in the older age group (significantly less than 1.0 in Toronto, Boston, New York, and Brighton). CONCLUSION: Homeless women under age 45 have extremely high levels of excess mortality, but older homeless women experience much milder levels of excess mortality. The usual survival advantage associated with being female is greatly attenuated among younger homeless women; in Toronto, New York, Philadephia, and Brighton, their death rates are not significantly different from that of homeless men. In contrast, older homeless women retain a large survival advantage over homeless men. Efforts to reduce deaths among homeless women should focus on prevention and treatment of HIV infection and drug overdose among those under the age of 45 years.
BRIEF QUESTIONS TO DETECT INADEQUATE HEALTH LITERACY AMONG VA PATIENTS. L.D. Chew ${ }^{1}$, K.A. Bradley ${ }^{2} ;{ }^{1}$ VA Puget Sound Health Care System, Seattle, WA; ${ }^{2}$ University of Washington, Seattle, WA (Tracking ID \#76683)

BACKGROUND: Many patients are unable to read and understand important health information. Providers are often unaware of patients' reading abilities and no brief effective screening test for limited health literacy exists. We evaluated and compared the performance of 16 potential literacy-screening questions for detecting inadequate or marginal health literacy to a standard health literacy instrument.

METHODS: We interviewed English-speaking patients seen at a VA preoperative clinic. The interview included 16 literacy-screening questions with scaled response options $(0-4)$, followed by a validated health literacy instrument, the Short Test of Functional Health Literacy in Adults (STOFHLA). Based on the STOFHLA, patients were classified as having inadequate marginal, or adequate health literacy. We calculated the sensitivity, specificity, positive and negative likelihood ratios, and area under receiver operating characteristic curves (AUROCs) for each literacy-screening question. The primary comparison standards were (1) inadequate health literacy and (2) inadequate or marginal health literacy, based on the STOFHLA. RESULTS: Of 332 participants, the mean age was 58.2 years and $95 \%$ were male. Based on the STOHFLA, $4.5 \%(\mathrm{~N}=15)$ of patients had inadequate health literacy and $7.5 \%(\mathrm{~N}=25)$ had marginal health literacy. For detecting inadequate health literacy, the 3 strongest screening questions were "How often do you have someone help you read hospital materials?" (HELP READ), "How often do you have problems learning about your medical condition because of difficulty understanding written information?" (PROBLEM LEARN), and "How confident are you filling out forms by yourself?" (CONFIDENT WITH FORMS) with AUROCs of 0.87 (95\%CI: $0.78-0.96), 0.80$ (95\% CI: $0.66-0.93$ ), and 0.76 (95\% CI: $0.62-0.90$ ), respectively. At a threshold of "Sometimes", HELP READ had a sensitivity of $73 \%$ and specificity of $83 \%$ of detecting inadequate health literacy $(+\mathrm{LR}=4.3,-\mathrm{LR}=0.32$ ), while at a threshold of "Occasionally", PROBLEM LEARN had a sensitivity of $79 \%$ and specificity of $65 \%(+\mathrm{LR}=2.26,-\mathrm{LR}=0.69)$. For CONFIDENT WITH FORMS, a cutoff of "Somewhat" identified $80 \%$ of patients with inadequate health literacy and screened $23 \%$ with adequate or marginal health literacy falsely positive $(+\mathrm{LR}=3.51,-\mathrm{LR}=0.26)$. The performances of all 16 questions were weaker for identifying patients with inadequate or marginal health literacy and combining questions resulted in no meaningful improvement in their screening performance. CONCLUSION: Although the screening performances of these 3 questions need to be confirmed in other populations, these questions may be effective in identifying VA patients with inadequate health literacy and alerting health care professionals to those patients for whom special methods of communication may be needed.

"HEAT IN THEIR INTESTINE": COLORECTAL CANCER PREVENTION BELIEFS AMONG CHINESE AMERICANS OVER 50. J.H. Choe ${ }^{1}, \mathrm{~J}_{\text {. } \mathrm{Lim}^{1}}, \mathrm{~N} . \mathrm{Burke}^{2}, \mathrm{E} . \mathrm{Acorda}^{3}$ V.M. Taylor ${ }^{3}$, S. Tu ${ }^{1} ;{ }^{1}$ University of Washington, Seattle, WA; ${ }^{2}$ University of California, San Francisco, CA; ${ }^{3}$ Fred Hutchinson Cancer Research Center, Seattle, WA (Tracking ID \#76409)

BACKGROUND: Asians have been among the fastest growing and most culturally diverse minority groups in the United States. However, there is relatively little detailed information regarding disease prevention behaviors and attitudes among various Asian American populations. As part of a larger project to increase cancer screening among Chinese Americans, the purpose of our study was to explore the knowledge, attitudes, and beliefs of older Chinese American patients toward colorectal cancer (CRC) screening modalities such as fecal occult blood testing (FOBT).

METHODS: We conducted 30 qualitative interviews focusing on CRC and health-seeking behavior among Chinese patients. Participants were male and female primary care patients older than 50 years of age recruited from the International Community Health Services, a culturally and linguistically appropriate neighborhood medical clinic centrally located in Seattle's Chinese American population. Trained bilingual and bicultural interviewers conducted audiotaped interviews in Mandarin or Cantonese, and then later translated these interviews verbatim into English. Qualitative software (N5/NUD*IST) was used to assist data management and analysis of transcripts. Transcripts were reviewed and discussed by six members of the research team for emerging themes, new information, and relationships among concepts.

RESULTS: Participants in our study often expressed a holistic view of health when asked about CRC prevention; generally, successful health promotion was defined as maintenance of positive "qi" (energy) and balanced "jing shen" (spirit) through exercise and diet moderation. Until prompted by interviewers, participants usually did not mention FOBT, flexible sigmoidoscopy, or colonoscopy while discussing CRC prevention. Many participants also believed in a causal pathway that differed from biomedical models of CRC causation (i.e. colon polyps as precursor lesions). These participants believed that unhealthy diet leads to gastrointestinal complaints such as constipation, and that the resultant rise in fecal "toxins" or "heat" leads to CRC. This belief in constipation causing CRC may have led to participants presuming that a lack of gastrointestinal symptoms renders them safe from CRC. This may also have led to participants believing other non-FOBT stool tests were forms of cancer screening.

CONCLUSION: Chinese American patients in our study expressed beliefs of health and CRC that differed from "Western" biomedical concepts. Failure by physicians to recognize these important different ideas of health promotion and CRC causation may create confusion among elderly Chinese American patients. Health promotion programs to increase CRC screening must incorporate these concepts to improve cultural relevance among Chinese American patients.

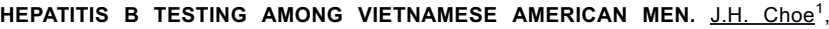
V.M. Taylor ${ }^{2}$, Y. Yasui ${ }^{2}$, N. Burke ${ }^{3}$, T.T. Nguyen ${ }^{3}$, E. Acorda ${ }^{2}$, J.C. Jackson ${ }^{1} ;{ }^{1}$ University of Washington, Seattle, WA; ${ }^{2}$ Fred Hutchinson Cancer Research Center, Seattle, WA; ${ }^{3}$ University of California, San Francisco, CA (Tracking ID \#76429)

BACKGROUND: Hepatocellular carcinoma (HCC) occurs more frequently among Asian immigrant populations than other racial/ethnic groups in the U.S., with highest incidence rates in Vietnamese American men (more than 12 times higher than non-Latino white men). This 
excess risk can be attributed to high rates of hepatitis B viral (HBV) infection and low rates of $\mathrm{HBV}$ vaccination. Identification of chronic viral carriers and vaccination of their unexposed family members can potentially reduce HCC incidence and mortality in Vietnamese Americans. The purpose of our study was to examine factors associated with HBV serology testing among Vietnamese American men.

METHODS: We conducted a population-based survey of randomly selected Vietnamese men living in Seattle. Trained bilingual and bicultural male interviewers conducted in-person surveys among adult men between 18-64 years of age. Our primary outcome measure was selfreport of any prior HBV serology testing. We used bivariate comparisons to study significant sociodemographic and health care predictors of HBV testing; a summary multi-variable model was constructed using stepwise logistic regression.

RESULTS: The survey was completed by 509 Vietnamese American men, with $79 \%$ response rate. Sixty-five percent of respondents reported any past serology test for HBV. In bivariate comparisons, the following variables were associated with past HBV testing $(\mathrm{p}<0.05)$ : age; marital status; proportion of life in the U.S.; English proficiency; health insurance type; having a regular source of care and a regular medical provider; self-reported health status; number of physician visits; reported long waits for medical appointments; having difficulty taking time off from work; and being concerned with medical costs. In our stepwise logistic regression model, the following factors were associated with any past $\mathrm{HBV}$ testing: older age $(\mathrm{OR}=2.5$ for over 50 compared to younger than $30 ; 95 \% \mathrm{CI}=1.1-5.5)$; college education $(\mathrm{OR}=2.5$ for college compared to high school education; $95 \% \mathrm{CI}=1.5-4.3)$; low English proficiency $(\mathrm{OR}=$ 2.5 compared to high English proficiency; $95 \% \mathrm{CI}=1.3-4.7)$; private health insurance $(\mathrm{OR}=$ 1.8 compared to public insurance; $95 \% \mathrm{CI}=1.0-3.4)$; having a non-Vietnamese regular medical provider $(\mathrm{OR}=5.3$ compared to having no regular provider; $95 \% \mathrm{CI}=2.9-10.0)$; and reporting no long waits for medical appointments $(\mathrm{OR}=2.2 ; 95 \% \mathrm{CI}=1.3-3.7)$.

CONCLUSION: Despite the high incidence of HCC in this population, more than one-third of Vietnamese men in our survey reported no history of HBV serology testing. In particular, younger and less educated men, those without private health insurance, and those without a regular medical provider are at particular risk for never having received serologic HBV testing. Educational programs to diminish the high rates of HCC should make special effort to target these vulnerable groups of Vietnamese Americans.

PERCEIVED TRUST AMONG VIETNAMESE-AMERICAN PATIENTS. S.P. Chu ${ }^{1}$, T.C. Collins ${ }^{2}$, A. Tranh ${ }^{3}$, B. Sharf ${ }^{4}$, K. O'Malley'; ${ }^{2}{ }^{1}$ Veterans Affairs Medical Center, MIRECC, HSR\&D, Houston, TX; ${ }^{2}$ Baylor College of Medicine, Houston, TX; ${ }^{3}$ University of North Carolina at Chapel Hill, Chapel Hill, NC; ${ }^{4}$ Texas A\&M University, College Station, TX (Tracking ID \#75774)

BACKGROUND: Studies have documented ethnic disparities in health care, but few studies have examined what factors contribute to these disparities, particularly between VietnameseAmericans and Caucasians. A possible factor may be issues of distrust between VietnameseAmericans and their health care providers. The objective of this study was to explore Vietnamese-Americans' feelings of trust for their providers, medical facilities, and the U.S. health care system.

METHODS: We recruited 24 Vietnamese-Americans stratified by type of health system (public, private, Veterans Affairs) who reported interaction with multiple levels of the system over the past year. Three trained moderators conducted four in-depth focus group interviews. We audiotaped and transcribed all of the interviews. All authors read the transcripts and documented initial impressions using a grounded theory approach. An analysis team of three authors discussed these initial impressions and devised a coding scheme based upon themes emerging from the data. This coding scheme was then presented back to the entire project team for further discussion and revision.

RESULTS: Similar to previous studies on factors that contribute to patient trust, VietnameseAmericans reported that trust is facilitated when waiting time is kept to a minimum ["Trust is the waiting room, period."], patient preferences are met ["if I see a female doctor, I could suddenly feel better."], and providers are competent [“I don't trust them .You're a student"]. Compared to previous findings on other ethnic groups, unique themes contributed to the development of trust among Vietnamese-Americans. Vietnamese-American patients value the professional conscience of the doctor ["If you love people, then you develop a responsibility towards the patients you treat and that is important"] and report themes of perceived discrimination ["if the medical personnel who deal with them had a kind of prejudice toward the races, they would treat one of the races better.'].

CONCLUSION: Among Vietnamese-Americans, trust was facilitated through keeping waiting time to a minimum, having patient preferences met, and being seen by a competent professional. Unique trust factors also emerged from this group-Vietnamese-American patients believed that the professional conscience of the doctor is important and that discrimination negatively impacts health care delivery. By identifying themes that lead to the development of trust between patients and physicians, we will make progress towards eliminating health care disparities for this fast growing population.

\section{MAJOR DEPRESSION AMONG MINORITY PATIENTS WITH OSTEOARTHRITIS:} PREVALENCE AND RESPONSE TO PAIN TREATMENT. $\underline{H}$. Chung ${ }^{1}$, T. Quinn ${ }^{1}$, P. Bhadra ${ }^{1}$, K. Barht ${ }^{1} ;{ }^{1}$ Pfizer, Inc., New York, NY (Tracking ID \#74561)

BACKGROUND: Previous studies of depression in patients with osteoarthritis have been limited by small samples and the lack of diagnostically valid measures. The goal of this analysis was to evaluate the prevalence of major depression among ethnic minority patients with osteoarthritis before they were enrolled in a pain treatment trial.

METHODS: African American, Hispanic, and Asian patients with osteoarthritis of the knee were enrolled in three separate and identically designed six week multicenter, randomized, double-blind parallel controlled studies with flexible dosing of celecoxib, naproxen, or placebo. The targeted enrollment for each study was 300 patients. The PHQ-9, a 9 item validated measure for depression assessment in primary care settings was used at screening evaluation. Scores $>10$ have a $88 \%$ sensitivity and specificity for DSM-IV major depression. Preliminary prevalence analyses with over $85 \%$ of the target enrollment are cited below.
RESULTS: 294 African Americans, 288 Hispanics, and 255 Asians were available for analysis. The mean PHQ-9 by group ranged from 3.1 (sd 4.3) to 6.2 (sd 5.5), with Hispanics having the highest mean score. Using a PHQ-9 cut off score of $>=10,23.8 \%$ of African Americans, $22.9 \%$ of Hispanics, and $10.2 \%$ of Asians had major depression. Among patients that reported any depressive symptoms, $50.7 \%$ of African Americans, $45.8 \%$ of Hispanics, and $32.5 \%$ of Asians reported that the symptoms made it difficult to carry out normal daily functions.

CONCLUSION: Major depression is highly prevalent among minority patients with osteoarthritis and is associated with functional burden. Clinicians are advised to screen for depression among these patients and monitor their symptoms during pain treatment

INVESTIGATOR BELIEFS AND REPORTED SUCCESS IN RECRUITING MINORITY SUBJECTS. G.M. Corbie-Smith ${ }^{1}$, D. St. George ${ }^{2}$, C. Blumenthal ${ }^{1}$; ${ }^{1}$ University of North Carolina, Chapel Hill, NC; ${ }^{2}$ Walden University, Minneapolis, MN (Tracking ID \#76225)

BACKGROUND: There is a growing literature on subject factors that influence recruitment of minority subjects, but little data on investigator factors that might influence success in minority recruitment. We sought to determine whether investigator beliefs are associated with perceived success in recruitment of minority subjects.

METHODS: Between August and October 2002, we surveyed by mail all National Heart, Lung and Blood Institute principal investigators (PIs) with at least one active study in 2001 and at least one study involving behavioral or biomedical human subjects research.

RESULTS: We analyzed data from the first 353 responding principal investigators (91\% White, $74 \%$ male, mean age $=50.4 \mathrm{y}(\mathrm{sd}=8.1)$. Most PIs $(67 \%)$ believed that inclusion of $\mathrm{racial} / \mathrm{ethnic}$ minority groups was very important to their research $(21 \%$ moderately important, $9 \%$ not very important and $4 \%$ not important at all). Most PIs believed that minority recruitment was very/somewhat difficult (range: $61 \%$ for recruitment of Asian Americans to $87 \%$ for American Indians/Alaska Natives vs. $22 \%$ for White Americans). PIs identified lack of information about effective recruitment strategies (76\%), participants' distrust (93\%), lack of access to the study population (78\%), transportation $(87 \%)$, dependent care $(81 \%)$, and cultural differences $(83 \%)$ as barriers to minority recruitment. In addition, PIs reported hiring minority staff in order to recruit minorities; $43 \%$ hired minority recruiters, $38 \%$ hired minority investigators, $16 \%$ added minority consultants, and $35 \%$ hired minority project managers. $73 \%$ of PIs felt strongly that they had been very successful in minority recruitment. In bivariate analyses PI race/ethnicity $(\mathrm{p}=.05)$, perceived importance of minority inclusion in their research area $(\mathrm{p}<.01)$, perceptions of barriers to recruitment $(\mathrm{p}<.01)$, and hiring of minority recruiters $(\mathrm{p}<.01)$, minority investigators $(\mathrm{p}<.01)$ and minority project managers $(\mathrm{p}<.01)$ were all associated with reported success. However, perceived difficulty of minority recruitment was not related to reported success in recruitment. In stepwise logistic regression modeling those PIs who believed more strongly in the importance of minority inclusion in their research area $(\mathrm{p}<.01)$, who hired minority investigators to increase minority recruitment $(\mathrm{p}=.01)$, and who perceived fewer barriers to recruitment reported more success $(\mathrm{p}<.01)$. CONCLUSION: While the majority of investigators noted difficulty in recruitment of minorities, those who identified fewer barriers to recruitment were most likely to report success. PIs who endorsed the importance of including minority subjects in their research and included minority investigators on the research team were more likely to report success. These findings could inform interventions to improve success in minority recruitment.

RACE IN THE GAIL MODEL OF BREAST CANCER. R. Crichlow ${ }^{1}$, J. Melnikow ${ }^{1}$, J. Nuovo ${ }^{1}$; ${ }^{1}$ University of California, Davis, Sacramento, CA (Tracking ID \#75958)

BACKGROUND: The Breast Cancer Risk Assessment (BCRA) Program (based on the Gail model) is advocated for risk screening for chemoprevention and determining eligibility for breast cancer studies. This model has never been validated in an African-American population. Objectives: To review the development and validation of the Gail model, to evaluate the impact of risk estimates on entry of African-American women into a prevention study, and examine the effect of ethnicity on the estimated breast cancer risk for hypothetical subjects.

METHODS: Design: Literature review of development and validation of the BCRA program, analysis of data from an interview study, and evaluation of BCRA with hypothetical subjects. Participants: 103 African-American and 632 non African-American women. Intervention: Women were screened with the BCRA program. Eligibility required an estimated 5-year risk of at least $1.7-\%$. Measures: Breast cancer risk was computed with race/ethnicity designated as "White" and then as "African-American" to determine eligible subjects. Hypothetical subjects with identical risk profiles were assessed as "African-American" and then "White" to assess the isolated effect of ethnicity on risk estimates.

RESULTS: BCRA program relative risks are originally from a study of 6000 White women, and the hazard rate calculation for African-Americans was based 128 cases. Using the BCRA program, all women were entered as "White" and $44.3 \%$ were eligible. Only $16.1 \%$ were eligible when all entered as "African-American." Designation as African American reduced our eligible subject pool by two-thirds.

CONCLUSION: Assessment of breast concerns by the BCRA program may be inaccurate for African-American women. This may lead to in appropriate recommendations on use of chemoprevention and disparities in recruitment for breast cancer prevention trials.

PRESCRIPTION FILL RATE AT A COMMUNITY HEALTH CENTER. C.M. Daniel ${ }^{1}$, M.A. Medow ${ }^{1}$, D.A. Wray ${ }^{1}$; ${ }^{1}$ Ohio State University, Columbus, OH (Tracking ID \#74465)

BACKGROUND: Medication adherence is of critical importance in disease management, especially in uninsured populations with an increased burden of morbidity. To our knowledge, there are no reported studies that measure the percentage of filled prescriptions by matching written prescriptions at the point of origin against pharmacy claims databases. We measured the percentage of written prescriptions that were filled by patients cared for at a community health center.

METHODS: A retrospective study of filled prescriptions for a 3-month period (JulySeptember, 2001). The health center subsidizes prescriptions for patients who are uninsured, not covered by programs that cover medication costs such as Medicaid, and are below the 
federal poverty income level. These patients can fill a month's supply of medication for a \$7 copay. We compared the clinic's carbon copies to claims data obtained from the pharmacy benefits manager. A prescription was considered filled if it was dispensed from the pharmacy within a 38-day period from the date of the prescription. We excluded insured patients, medications obtainable through assistance programs, non-medication prescriptions, over-thecounter medications and insulin.

RESULTS: 299 unique patients had 574 prescriptions written, out of which 383 were filled ( $66.7 \%, 95 \%$ confidence interval $62.7 \%$ to $70.6 \%)$. The median time to fill the prescription was 2 days (interquartile range $0-12$ days).

CONCLUSION: Prescription fill rates in this population are suboptimal despite a low copayment. Barriers to filling prescription drugs are apparent for uninsured patients at or below the poverty level despite subsidized drug coverage. Future studies of patient, medication and disease characteristics that affect adherence are warranted in indigent populations, especially since the number of uninsured patients are sizable and rising.

HEALTH PROMOTION CLASS IN THE SOUTH BRONX: AN ACADEMIC HEALTH CENTER-COMMUNITY PARTNERSHIP MODEL. D. Daniels ${ }^{1}$, M. Venegas-Pizarro ${ }^{1}$, L. Smith ${ }^{2}$; ${ }^{1}$ Residency Program in Social Medicine, Montefiore Medical Center, Bronx, NY; ${ }^{2}$ Residency Programs in Social Medicine and Primary Care, Montefiore Medical Center, Bronx, NY (Tracking ID \#75388)

BACKGROUND: From Dutch to Dominicans, since the 1600s Highbridge has been home to immigrants. In the 1960 s and 70 s, a massive, multifactorial economic decline led to street gangs, crack houses, low real estate values and high crime rates in the South Bronx. Today, the $58 \%$ Latino and 36\% African American community is slowly rebuilding. Even so, New York City health statistics from the late 90 s show Highbridge with the city's worst asthma, diabetes, HIV and TB complication rates. We are using a non-traditional model to address these issues. OBJECITVES: 1 . To form an equitable, sustainable partnership between a community [Highbridge], a community center [Highbridge Community Life Center (HCLC)] and an Academic Health Center (AHC) [Montefiore Medical Center (MMC)]. 2. To improve health education and access in Highbridge. 3. To create a reproducible model. 4. To allow medical student and resident interaction in communities they serve in order to to improve understanding and breakdown racial barriers.

METHODS: 1. Find a trusted community organization (church, school, community center) in an area served by the AHC. 2. Hold focus groups and attend meetings with organization staff and other community leaders to ascertain their views on the community's health, unmet health needs, how to meet health needs and if they envision a partnership with the AHC to help meet these needs. 3. Ask to participate in community meetings (tenants' meetings, parents' groups, youth outreach) and hold specific health meetings in order to ask the community the same questions. 4. Implement ideas set forth in the above meetings. 5. Establish an 'official,' sustainable outreach. 6. Stress the need for a primary doctor and invite students to our South Bronx clinic.

RESULTS: The CPM has resulted in a trusting relationship between the three members, a Health Promotion Class (HPC) and improved access to health care. The bilingual HPC is a community-led, educational offering of the HCLC. This class, taught by residents, uses a biopsycho-sociopolitical model of illness, focuses on prevention, addresses social justice issues and encourages action.

CONCLUSION: The CPM has allowed us to form a strong relationship with our community and may serve as model for other AHCs. With mutual consent, we want to conduct research on the effectiveness of the CPM in Highbridge. More and more, AHCs may turn to nontraditional methods in order to improve health outcomes in the communities they serve.

RACIAL DIFFERENCES IN THE KNOWLEDGE OF BREAST CANCER RISK FACTORS. $\underline{\text { S. Davids }}{ }^{1}$, M. Schapira ${ }^{2} ;{ }^{1}$ Medical College of Wisconsin, Milwaukee, Wl; ${ }^{2}$ Medical College of Wisconsin, Shorewood, WI (Tracking ID \#76858)

BACKGROUND: We and others have previously reported inaccurate perceptions of breast cancer risk. Prior studies have also demonstrated a misunderstanding of breast cancer risk factors. Poor breast cancer risk factor knowledge could be associated with inaccurate breast cancer risk perceptions.

METHODS: As part of a clinical trial examing breast cancer risk perceptions, subjects were surveyed regarding knowledge of breast cancer risk factors. The factors included those used in the Gail model, as well as smoking, alcohol, diet and bruising to the breast. Perceptions for each risk factor were tabulated and stratified by race and compared using a chi square analysis. RESULTS: Of the 254 women, $32 \%$ were of non-white race, and $93 \%$ of those were African american. African american women were younger, had less years of education, and a lower income than the white women. They also overestimated their lifetime risk of breast cancer to a greater degree than white women (mean $35.4 \%$ vs. $27.4 \%, p=0.01$ ). The majority of women from both groups incorrectly identified smoking and a high fat diet as a risk factor in the development of breast cancer. In addition, the majority of both groups did not identify that drinking more than 2 alcoholic beverages per day, or having a child at age $>30$ are breast cancer risk factors. Knowledge of other factors differed between groups (table).

CONCLUSION: Overall, women have a poor knowledge of established breast cancer risk factors and especially in women of African american race. Correcting this knowledge deficit may improve the accuracy of breast cancer risk perceptions.

Racial Differences: Perceived Breast Cancer Risk Factors

$\begin{array}{lccc} & \text { African American } & \text { White } & \text { p-value } \\ \text { Age at menses }<10 \text { yrs } & 27 \% & 42 \% & 0.02 \\ \text { Increasing age } & 47 \% & 80 \% & <0.001 \\ \text { Nulliparous } & 12 \% & 52 \% & <0.001 \\ \text { Family history of breast cancer } & 74 \% & 95 \% & <0.001 \\ \text { Breast trauma } & 69 \% & 31 \% & <0.001\end{array}$

ETHNICITY, ACCULTURATION, AND ALCOHOL USE AMONG HISPANIC PATIENTS IN PRIMARY CARE. N.V. Dawson ${ }^{1}$, R.D. Cebul ${ }^{2}$, D. Einstadter ${ }^{3}$, T.V. Parran ${ }^{4}$, R.C. McCormick ${ }^{5}$, S. Kanuch ${ }^{6}$, C.L. Thomas ${ }^{1}$, N. Cofresi ${ }^{7}$, J.J. Sudano ${ }^{1}$; ${ }^{1}$ Case Western Reserve University, Cleveland, OH; ${ }^{2}$ Case Western Reserve University, Hunting Valley, OH; ${ }^{3}$ Case Western Reserve University, Rocky River, OH; ${ }^{4}$ Case Western Reserve University, Shaker Heights, $\mathrm{OH} ;{ }^{5} \mathrm{Case}$ Western Reserve University, Hudson, $\mathrm{OH} ;{ }^{6}$ MetroHealth Medical Center, Cleveland, $\mathrm{OH} ;{ }^{7}$ Cleveland State University, Cleveland, $\mathrm{OH}$ (Tracking ID \#76638)

BACKGROUND: There is conflicting evidence about the relationships among ethnicity, acculturation, and alcohol use. In this study, we compare the results of English and nonEnglish speaking primarily Puerto Rican Hispanics on a standardized alcohol-screening instrument.

METHODS: Subjects were Hispanic patients with established primary care relationships in a General Medicine practice at a large urban teaching hospital. The Alcohol Use Disorders Identification Test (AUDIT), CAGE, and sociodemographic questions were administered using an Interactive Voice Response (IVR) interviewing system. Patients chose either the English or Spanish version of the survey. Relationships between AUDIT scores, version of the survey, and patients' characteristics were evaluated using censored regression (Tobit) modeling (appropriate when the dependent variable follows a truncated normal distribution).

RESULTS: Of 355 patients, $242(68 \%)$ selected the English version and 113 selected the Spanish version of the AUDIT IVR screen. In bivariable analyses, Spanish speaking patients tended to be older, less educated, less likely to be currently employed, and were more likely to have lower incomes, and to have Medicaid or Medicare insurance. 128 patients taking the English version (53\%) and 71 patients taking the Spanish version (63\%) had AUDIT scores of zero $(\mathrm{p}=.08)$. CAGE scores were not different between English and Spanish speaking Hispanics $(\mathrm{p}=.7)$. In the Tobit model, lower AUDIT scores were associated with older age and female gender; higher AUDIT scores were associated with being a smoker, reporting less frequent spiritual activity, and having higher personal CAGE scores. After adjusting for other variables in the Tobit model, screening in English (vs. Spanish) was not associated with AUDIT scores.

CONCLUSION: Acculturation, as represented by language of choice, appears not to influence alcohol screening scores among Puerto Rican Hispanic patients seen in primary care. Regardless of acculturation, alcohol use was most influenced by age, gender, smoking status, reported level of spiritual activity, and CAGE scores.

IN SEARCH OF MEDICAL CARE: THE DIMINISHING AVAILABILITY OF PUBLICLY FINANCED SERVICES. A.L. Diamant ${ }^{1}$; R.D. Hays ${ }^{1}$; S. Asch ${ }^{2}$; L. Morales ${ }^{1}$; D. Calmes ${ }^{3}$; A. Saravia ${ }^{1}$; E. Fielder ${ }^{1}$; G. Sumner ${ }^{1}$; I. Dyer ${ }^{4}$; J. Fielding ${ }^{4}$; G. Lillian ${ }^{1} ;{ }^{1}$ University of California, Los Angeles, Los Angeles, California; ${ }^{2}$ RAND, Santa Monica, California; ${ }^{3}$ Charles R. Drew University, Long Beach, California; ${ }^{4}$ Los Angeles County Department of Health Services, Los Angeles, California. (Tracking ID \#76638)

BACKGROUND: Problems with access to health care appear to be worsening. Accessing health care is increasingly difficult for individuals who rely on publicly financed care, because of clinic closures due to financial shortfalls and cutbacks. This study evaluates what alternate sources of health care Los Angeles County patients would turn to if their current publicly financed clinic were closed.

METHODS: We interviewed a stratified cross-sectional probability sample $(n=2,030)$ from among all adult patients who received primary care services during Februrary through July, 2002 from the Los Angeles County Department of Health Services (LAC-DHS). Face-to-face interviews were performed at all four types of medical facilities operated or contracted with the LAC-DHS (Comprehensive Health Centers, Health Centers, Hospital Outpatient Clinics and Public/Private Partnership Clinics) and were distributed throughout Los Angeles County. The sample was racially and ethnically diverse (Latino/Hispanic 72\%, African American 11\%, nonHispanic white $10 \%$, Asian/Pacific Islander $4 \%$, and Other $3 \%$ ); almost two-thirds were female; the mean age was 43 years; the median annual household income was between $\$ 5,001$ and $\$ 10,000$; and $64 \%$ of respondents were uninsured.

RESULTS: $78 \%$ of patients reported receiving medical care at a County facility within 12 months prior to the sampled visit, but only $53 \%$ overall reported having a usual source of care. When asked how likely it was that they would return to the same clinic where they were interviewed if they had the option to go anywhere for medical care $73 \%$ reported very likely, $15 \%$ said somewhat likely, $4 \%$ were uncertain, and $8 \%$ were somewhat or very unlikely to return. When asked where they would seek medical care if the facility they were currently at were to close permanently nearly two-thirds $(64 \%)$ reported that they would go to another County facility, $10 \%$ indicated they would go to a non-County facility, $10 \%$ to an emergency department, $8 \%$ did not know where they would go, $3 \%$ would go home or do nothing, $1 \%$ would go to Mexico and $4 \%$ would do something else.

CONCLUSION: The majority of adult patients receiving primary care services through the LAC-DHS have received prior care in the system within the prior 12 months. The majority of these individuals report an expectation of future use of publicly financed care despite the downsizing of urban public health systems by clinic closures and reductions in services.

RACE AFFECTS LIPID-LOWERING MANAGEMENT IN HOSPITALIZED PATIENTS WITH CORONARY HEART DISEASE. D.D. Dressler ${ }^{1}$, H. Byrapuneni ${ }^{1}$, T. Jacobson ${ }^{1}$; ${ }^{1}$ Emory University, Atlanta, GA (Tracking ID \#75248)

BACKGROUND: The efficacy of lipid-lowering agents in reducing cardiac events and death in coronary heart disease (CHD) patients has been well-described. However, the prescription of these medications to eligible patients continues to lag behind national recommendations. While racial disparity in the delivery of certain therapies to CHD patients has been recently demonstrated, few studies have addressed racial differences in the prescription of lipidlowering therapies to hospitalized coronary heart disease patients. The purpose of this study is 
to determine the association between patient characteristics and the appropriate management of lipid-lowering therapy in CHD patients discharged from the hospital.

METHODS: A cohort of 258 consecutive patients admitted to a large, inner-city university hospital with a diagnosis suggestive of CHD was identified from admission records to the internal medicine service. Of these, 98 had documented CHD and were further evaluated for optimal versus suboptimal lipid management, an outcome evaluating intensification of lipidlowering therapy when indicated using the National Cholesterol Education Program (NCEP) guidelines for low density lipoprotein (LDL) cholesterol goal.

RESULTS: Overall, the rate of suboptimal lipid management during hospitalization was $48 \%$, while the rate of suboptimal lipid management in patients who were not at NCEP LDL goal was $75 \%$. Ethnicity was a significant predictor of suboptimal lipid management. Black CHD patients were significantly more likely to have suboptimal lipid management than non-black patients by the time of hospital discharge $(52.3 \%$ vs. $16.7 \%, \mathrm{p}=0.021)$. This result remained statistically significant in stratified analysis as well as multivariate logistic regression analysis controlling for clinically important potential confounders.

CONCLUSION: Based on the rates of suboptimal lipid management, clinicians need to improve their efforts in caring for hospitalized CHD patients. The disparity in clinical management of black CHD patients may help explain the differential in health outcomes seen between black and white patients. Future efforts should focus on encouraging evidence-based therapies for all CHD patients discharged from the hospital, independent of race.

GENDER DISPARITIES IN HAART USE AMONG HIV-INFECTED PATIENTS IN THE UNITED STATES. D.P. Eisenman ${ }^{1}$, C. Bird ${ }^{2}$, B. Collins ${ }^{2}$, R. Beckman ${ }^{2}$, W.E. Cunningham ${ }^{1}$; ${ }^{1}$ University of California, Los Angeles, Los Angeles, CA; ${ }^{2}$ RAND Corporation, Santa Monica, CA (Tracking ID \#74702)

BACKGROUND: Highly active antiretroviral therapy (HAART) is standard for HIV care since 1996 but its unknown if the same proportion of women and men are receiving HAART and the factors accounting for any disparity.

METHODS: We analyzed data from a longitudinal sub-sample of 1397 adults drawn from the HIV Costs and Services Utilization Study, a nationally representative sample of HIV-infected persons in care in 1996 and reinterviewed in late 1998. Members of the HCSUS sample were eligible if they were interviewed in English at HCSUS baseline, their gender was unambiguous based on HCSUS data, and they had participated in the second follow-up HCSUS interview. The sub-sample was weighted to represent the surviving members of the nationally representative sample. We calculated the percent of men $(n=893)$ and women $(n=504)$ who were eligible for HAART in 1998 based on 1996 clinical guidelines and the percent of eligibles that were receiving HAART. HAART was defined as certain combinations of nucleoside reverse transcriptase inhibitors plus certain protease inhibitors (PIs), combinations of PIs, or the combination of a PI plus a non-nucleoside reverse transcriptase inhibitors. We built separate logistic regression models of gender on current use of HAART in which age, race, education, income, and insurance status were singly added to the model. The effect of the added variable on the odds ratio (OR) and $95 \%$ confidence interval (CI) for female gender was ascertained. A final model included gender, HAART and all variables that were significant predictors in the separate models.

RESULTS: Almost all men and women were eligible for HAART $(99.9 \%$ of women eligible vs $99.3 \%$ of men, $\mathrm{p}=0.35$ ), but women were less likely to receive HAART than men. Only $47.6 \%$ of eligible women were currently receiving HAART compared to $57.6 \%$ of men ( $\mathrm{p}=$ $0.005)$. Gender differences were accounted for by education and income, each of which was strongly, positively and independently related to receipt of HAART (OR for college graduate vs. less than high school education $1.8,95 \%$ CI $1.1-3.1, p=.02$; OR for income $>\$ 25,000$ receiving HAART $1.895 \% \mathrm{CI} 1.3-2.7, \mathrm{p}=.002$ ) and each of which was lower among women than men ( $4 \%$ of women were college graduates vs $26.4 \%$ of males; $10.8 \%$ of women had incomes $>\$ 25,000$ vs. $36.2 \%$ of males). Together, income and education changed the gender OR from $0.7, p<.001$ to $1.2, p=0.4$. Gender differences in HAART were not influenced by differences between men and women in the distribution of age, race, insurance status, CD4 count, AIDS stage, or region of the U.S.

CONCLUSION: Gender disparities in HAART use were prevalent and were explained by the relative lack of resources for women with HIV/AIDS. To reduce gender disparities in HIV care, policies are needed to provide additional resources to women.

PHYSICIAN SPANISH LANGUAGE ABILITY, CULTURAL COMPETENCE, AND THE EXPERIENCES OF CARE OF SPANISH-SPEAKING PATIENTS. A. Fernande ${ }^{1}$, D. Schillinger ${ }^{1}$, K. Grumbach ${ }^{1}$, A. Rosenthal ${ }^{1}$, F. Wang ${ }^{1}$, E.J. Perez-Stable ${ }^{1}$; ${ }^{1}$ University of California, San Francisco, San Francisco, CA (Tracking ID \#75778)

BACKGROUND: Physicians' language ability and cultural competence are often cited as important in the care of non-English speaking patients, yet there are few studies examining how language barriers affect health communication and no studies linking cultural competence with patients' experiences of care. We studied physician patient dyads to determine how physician self-rated Spanish-language ability and cultural competence affect Spanish-speaking patients' reports of interpersonal processes of care.

METHODS: Questionnaire study of 116 monolingual Spanish-speaking patients with Type 2 diabetes and 48 primary care physicians (PCPs) at a public hospital with interpreter services. PCPs rated their Spanish ability on a 5 point Likert scale and rated their understanding of the health related cultural beliefs of their Spanish-speaking patients on a 4 point scale. We assessed patients' experiences using scales from the validated Interpersonal Processes of Care in Diverse Populations (IPC) instrument. PCP responses were dichotomized, as were IPC scale scores (optimal vs. non-optimal). We analyzed the relationship between language and IPC, and cultural understanding and IPC, adjusting for PCP gender,level of training, and patient age, gender, education, years with PCP, years with diabetes, and clustering of patients by PCP. RESULTS: 26 (54\%) PCPs reported "excellent/good" Spanish ability, 22 (45\%) reported "fair/poor/no" ability. 40 (83\%) PCPs understood the health related cultural beliefs "very or somewhat well"; 8 (17\%) "not very/not at all well". Language ability and cultural understanding were moderately correlated (Spearman correlation 0.55). Greater Spanish ability was strongly associated with optimal IPC score across $2 / 5$ domains: elicitation of patient concerns (AOR 5.56, p = 0.003) and explanation of condition (AOR 2.37, p = 0.05), and marginally associated with patient empowerment $(\mathrm{AOR}=2.23, \mathrm{p}<0.09)$ but not with IPC scores in the domains of general clarity or explanations of process of care. Greater understanding of health related cultural beliefs was associated with the same three IPC domains (eg, elicitation of concerns: AOR 11.63, p = 0.01) but weakly associated with general clarity and not associated with explanations of process of care.

CONCLUSION: Physician self-rated language ability and cultural competence are independently associated with patients' reports of interpersonal processes of care, particularly in non-technical domains that may be less amenable to professional interpretation. Our study provides empirical support for the role of language and cultural competence in the primary care of non-English speaking patients.

PHYSICAL COMPLAINTS OF TORTURE SURVIVORS: ATTRIBUTION TO PRIOR VIOLENT ACTS. A. Finlay ${ }^{1}$, C. Meserve ${ }^{1}$, T. Comerci ${ }^{1}$, H. Watanabe ${ }^{1}$, B. Rosenfeld ${ }^{2}$, A. Keller ${ }^{1} ;{ }^{1}$ New York University, New York, NY; ${ }^{2}$ Fordham University, New York, NY (Tracking ID \#75763)

BACKGROUND: As the United States has become host to increasing numbers of political refugees, inner city clinics are caring for a growing number of patients with histories of torture. In order to understand their health problems, we conducted a retrospective study of primary care visits at a municipal hospital clinic caring for torture survivors in New York City. We specifically sought to describe torture survivors' physical symptoms and to understand if specific symptoms bore any relationship to the torture they endured.

METHODS: We reviewed the medical charts of 157 patients randomly selected from the 278 patients seen at the clinic between January 1, 2000 and December 15, 2002. We collected and analyzed data on physical symptoms, demographics, and utilization of interpreter and mental health services. We grouped symptoms by organ system into 15 categories and documented whether patients attributed each symptom to their experience of torture.

RESULTS: Mean age was 33.5 years; $61 \%$ were men; $60 \%$ were from Africa, $25 \%$ from Asia, $6 \%$ from Latin America and $9 \%$ from other countries. Patients predominantly complained of musculoskeletal symptoms (61\%; most common: back pain, knee pain); neurologic complaints (51\%; headache, dizziness); sleep problems (46\%; insomnia, nightmares) and gastrointestinal symptoms (44\%; abdominal pain, rectal disorders). More than half $(55 \%)$ of the patients attributed at least one of their physical complaints to antecedent trauma. Musculoskeletal symptoms (MSK) and sleep problems were the two symptom categories that were more likely attributed to torture than not (sleep: $23.5 \%$ vs. $8.6 \%, \mathrm{p}=0.001$; MSK: $25.2 \%$ vs. $18.6 \%, \mathrm{p}=$ 0.04). Nearly $58 \%$ of patients received specialized mental health services for symptoms of anxiety, depression or post-traumatic stress disorder. A total of $53 \%$ of the patients required interpreters, most commonly: French (37\%), Tibetan (24\%) and Spanish (7\%).

CONCLUSION: The presenting complaints of these torture survivors fall within the spectrum of those seen in primary care clinics. However, a majority of these patients attributed at least one complaint to antecedent torture. These patients also have notable psychological and interpreter needs that may distinguish them from non-refugee patients. Adequate mental health and linguistic resources are necessary to fully serve this population. Future studies that longitudinally assess torture survivors' physical complaints with relation to history of violent acts, psychiatric comorbidity and type of treatment would be useful to most effectively address this group's health needs.

AMONG DIABETIC VETERANS, GLYCEMIC CONTROL IS ASSOCIATED WITH HISPANIC VERSUS NON-HISPANIC ETHNICITY. R.E. White ${ }^{1}$, L.R. Flores ${ }^{1} ;{ }^{1}$ University of New Mexico, Albuquerque, NM (Tracking ID \#76218)

BACKGROUND: Because ethnic minority populations experience disproportionately higher rates of diabetes and its complications, we sought to discern whether race (Hispanic versus non-Hispanic White) could account for diabetes control differences, as measured by Hemoglobin A1c.

METHODS: All Albuquerque Veterans Affairs primary care patients with diabetes were segregated into thirds by their last HbA1c: 9.0 or higher, 7-8, or under 7. Random samples from each third produced 717 total subjects who were mailed surveys, seeking information about ethnicity, education level, ease of obtaining care, and participation in care. Age, the presence of co-morbid conditions, and all $\mathrm{HbA1C}$ measurements for one year were obtained from the computerized medical record for each respondent. Student's t-test was used to compare mean HbA1C between Hispanic and non-Hispanic White respondents. Linear regression was used to measure the strength of association of continuous variables. A generalized linear multivariate model was used to ascertain the simultaneous effect of several independent variables on $\mathrm{HbA1C}$. A p value of 0.5 was used to assess statistical significance; two-tailed tests were used throughout.

RESULTS: Surveys were returned by 403 patients: $59 \%$ from the under 7 A1c patient group, $58 \%$ from the $7-8 \mathrm{~A} 1 \mathrm{c}$ group, and $51 \%$ from the 9 or higher group. Further analysis was performed on the responses from those 136 patients who identified themselves as Hispanic, compared to 141 self-identified, non-Hispanic White patients. The two ethnic groups had similar mean ages $(67,69, \mathrm{p} \equiv 0.11)$ with a median age of 70 years (range 34-89). The mean HbA1C for Hispanic subjects was $8.3 \%$ and for non-Hispanic White subjects $7.7 \%$ representing a difference of $0.6 \%(95 \%$ confidence interval $0.2,1.0, \mathrm{p}<0.001)$. In multivariate analysis A1c values were independently associated with age $(\mathrm{p} \equiv 0.0002)$, needing medication for diabetes care $(p \equiv 0.0005)$, ethnicity $(p \equiv 0.0041)$, education $(p \equiv 0.0044)$, reliance on family members for diabetic care $(\mathrm{p} \equiv 0.0139)$, and tobacco use $(\mathrm{p}=0.0194)$.

CONCLUSION: For diabetic patients, HbA1c meaured glycemic control appears to be poorer for Hispanic than for non-Hispanic White veterans, even after accounting for other plausible influences. This supports the concern that ethnic minorities suffer more from diabetes. However, attention to ethnicity might improve treatment outcomes. 
DOES DIFFERENTIAL DIFFUSION OF TECHNOLOGIES HELP EXPLAIN RACIAL DISPARITIES IN CARDIAC CARE? A. Fremont ${ }^{1}$, S. Wickstrom ${ }^{2}$, M. Shah ${ }^{2}$, T. Horstman ${ }^{2}$, C. Bird ${ }^{1}$, J. Escarce ${ }^{1} ;{ }^{1}$ RAND Health, Santa Monica, CA; ${ }^{2}$ Center for Health Care Policy and Evaluation, Minneapolis, MN (Tracking ID \#76822)

BACKGROUND: Racial disparities in healthcare are well documented, particularly for cardiovascular care. Yet, little is known about why these disparities occur or change over time. We assess whether differential diffusion of cardiac technologies over time contributes to observed disparities.

METHODS: We obtained 7 years (1994-2000) of claims data from commercial health plans affiliated with UnitedHealthcare, representing 3.5 million enrollees in 2000. Annual cohorts were constructed based on enrollee age $(>45)$ and cardiac diagnoses for each of 8 technologies that vary in stage of diffusion: exercise stress test, radionuclide stress test, stress echo, coronary angiogram, and coronary angioplasty, artherectomy, stent and CABG. A previously validated measure of race was obtained by geocoding enrollees' address to Census data at the Blockgroup level. Diffusion curves for each racial subgroup (Black vs. NonBlack) were constructed to assess differential patterns of diffusion by technology and race. Chi-Square tests were used to compare unadjusted rates between cohorts and multiple logistic regression models were used to adjust for age, gender, SES, cardiac diagnoses, and plan.

RESULTS: Blacks were less likely than NonBlacks (Black-NonBlack $\mathrm{RR}<.82, \mathrm{P}<.05$ ) to receive 4 of 8 services including exercise stress test, radionuclide stress test, exercise echo, and cardiac stent, but the pattern varied substantially by year and technology. For example, exercise stress tests, a well established technology that was decreasingly used among NonBlacks over time, showed large racial disparities in 1994 (rate per 1000: 162 for Blacks vs. 253 for NonBlacks, $\mathrm{P}<.001$ ) that narrowed and became insignificant by 1998 (194 vs. $201, \mathrm{P}=.72$ ). In contrast, no disparities were apparent for stress echos until 1996 (27 vs. 47, $\mathrm{P}=.03$ ) when the technology began to rapidy diffuse in the plans considered; the gap persisted through 2000 ( 70 vs. $101, \mathrm{P}=.02$ ). Similarly, once cardiac stents began rapidly diffusing, a racial disparity developed favoring NonBlacks and remained significant for 1997-2000 (1997 rate: 107 vs. 181, $\mathrm{P}=.007 ; 2000$ rate: 173 vs. $252, \mathrm{P}<.001)$. Adjusting for covariates did not alter the basic pattern of results.

CONCLUSION: We found that racial disparities varied depending on the stage of diffusion of cardiac technologies with larger disparities observed for relatively new and rapidly diffusing technologies whereas disparities for more established technologies or ones with newer alternatives were smaller or not present. Researchers and policy makers should take stage of diffusion of services into account when planning or evaluating efforts to reduce disparities.

THE IMPACT OF ACCULTURATION ON PAP SMEAR PRACTICES OF HAITIAN WOMEN IN GREATER BOSTON. E.H. Green ${ }^{1}$, L. Ko ${ }^{1}$, N. Prudent ${ }^{1}$, M. Posner ${ }^{1}$, K.M. Freund ${ }^{1}$, M. David ${ }^{1}$; ${ }^{1}$ Boston University, Boston, MA (Tracking ID \#76131)

BACKGROUND: Delayed detection of cervical abnormalities may account for the higher cervical cancer mortality in minority women. One potential barrier to Papanicolaou (pap) smear acquisition in immigrant women is the degree to which they have become assimilated into American culture. We examined the impact of this process, also known as acculturation, on pap smear rates of Haitian women in greater Boston.

METHODS: We performed a cross-sectional survey of women 40 years old and over from randomly selected households in neighborhoods with high concentrations of Haitian women to explore cancer screening behaviors. Trained bilingual interviewers conducted in-person interviews using a standardized instrument to assess demographics, health care practices and beliefs, and acculturation. Our measures of acculturation were the language in which the survey was conducted, the percent of an individual's life spent living in the United States, and a series of 13 questions that quantified the preferred language used in common situations. We compared Haitian women reporting having had a pap smear within 3 years to other Haitian women. We created a model using stepwise logistic regression with acculturation, demographics, and other characteristics considered as predictors of pap smear acquisition.

RESULTS: In total 753 women (76\%) participated. Of these, 281 (37\%) reported their ethnicity as Haitian and had a known pap smear status. Pap smear rates were high $(80 \%$ [95\% CI $75 \%, 84 \%]$ ). In bivariate analysis, women were less likely to have had a pap smear if they used Haitian-Creole in common situations more than average $(87 \%$ vs. $68 \%, \mathrm{p}<.001)$. In multivariate analysis, however, this trend failed to reach statistical significance (adjusted odds ratio (AOR) .5 [95\% CI .2, 1.5]). Women who had lived less than $25 \%$ of their life in the US were more likely to have had a pap smear (AOR $4.7[1.7,13.0])$; however, in stratified analysis this trend remained statistically significant only for women less than 50 years old (AOR 43 [2.4, $1,000]$ for women $<50$, AOR $1.5[.43,5.0] \geq 50$ ). Important predictors of pap smear acquisition included knowledge of a pap smear (AOR 8.9 [3.5, 23]; having had a "check-up" visit (AOR 3.9 ; [1.3, 11.2]); being younger (age 40-49 AOR 15.1 [3.2, 71]; 50-59 AOR 13.6 [2.5, 73]; 6069 OR $5.1[1.2,21]$ all vs. $\geq 70$ ); having a mammogram within 2 years (OR 2.7 [1.2. 6.3]; having a female physician (OR 2.1 [1.2-3.6]); and being married (OR 1.8 [1.0-3.1]).

CONCLUSION: Conventional measures of acculturation do not seem to influence pap smear rates. There may be differences in the screening patterns of younger Haitian immigrants compared to older ones. Future efforts to improve cancer screening among immigrants may need to focus less on acculturation and more on education.

\section{FUNCTIONAL HEALTH LITERACY, COMORBIDITY AND HEALTH STATUS. C.E. Guerra ${ }^{1}$,} J.A. Shea ${ }^{1}$; ${ }^{1}$ University of Pennsylvania, Philadelphia, PA (Tracking ID \#75701)

BACKGROUND: Functional health literacy has been shown to be related to use of health services, knowledge of medical conditions and to some extent health outcomes: lower health literacy is often associated with poorer health status. This study was undertaken to evaluate the relationship between functional health literacy and 1) the presence of comorbidities, 2) physical health status, and 3) mental health status.

METHODS: This study was is part of a larger study assessing literacy and patient satisfacation. A convenience sample of patients over 18 years of age who were insured by either Medicaid or
Medicare and who were waiting to see their physicians at four community and one universitybased primary care practices were invited to participate. Overall 2863 patients were eleigible for the parent study: 1793 agreed to participate. A total of 1301 completed four key instruments for the present study: a demographics questionnaire, the Test of Functional Health Literacy in Adults (TOFHLA), the Charlson Comorbidity Index (CCI) and the SF-12, an assessment of health status comprised of physical health (PCS-12) and mental health (MCS12). Pearson correlation coefficients eatimated relationships between the TOFHLA, CCI, PCS-12 and MCS-12 scores. Separate stepwise regressions were conducted with the CCI, PCS-12 and MCS-12 scores as the dependent variables and TOFHLA score, sex, age, education, ethnicity (Hispanic or African American) and spoken language (English or Spanish) as independent variables.

RESULTS: The mean age of the 1301 respondents was 42.2 (s.d. $=15.1)$ years. The majority of participants were women ( $76 \%$ ) and Latino $(60 \%) .17 \%$ of the patients had less than an 8 th grade education. The mean TOFHLA score was 27.1 (s.d. = 9.1) out of 36 . The proportion of patients with 1 or more comorbidities was $36 \%$. Mean PCS-12 and MCS-12 scores were 41.3 (s.d. $=11.5$ ) and 43.9 (s.d. $=11.5$ ), respectively. Correlations between the TOFHLA score and health indicators were -0.21 ( $\mathrm{p}<0.0001$ ) with CCI, 0.16 ( $\mathrm{p}<0.0001)$ with PCS-12 and 0.05 $(p=0.08)$ with MCS-12. After adjusting for confounders, functional health literacy remained a significant positive predictor of CCI score $(p=.0006)$. Other positive significant predictors were being male $(\mathrm{p}=.02)$ and older age $(\mathrm{p}=.0001)$. Significant positive predictors of the PCS12 score were younger age $(\mathrm{p}=.0001)$ and higher education $(\mathrm{p}=.04)$ and of MCS-12 score were higher education $(\mathrm{p}=.0004)$.

CONCLUSION: Inadequate functional health literacy is associated with a greater comorbidity burden as measured by the CCI, even though the CCI is heavily biased towards inpatient illnesses. One might imagine that a more sensitive instrument that included common outpatient conditions such as hypertension would show an even stronger effect. It is somewhat surprising, though reassuring, that there was no association between functional health literacy and physical and mental health as measured by the SF-12, after adjusting for sociodemographic confounders. More study is clearly need to elucidate the strength and direction of the relationships between health literacy and a wide range of health outcomes.

COMMITMENT TO CARING FOR THE UNDERSERVED: A STUDY OF INTERNAL MEDICINE RESIDENTS' ATTITUDES OVER THE COURSE OF RESIDENCY. A.R. Gupta ${ }^{1}$, R. Horwitz ${ }^{1}$; ${ }^{1}$ Yale University, New Haven, CT (Tracking ID \#76760)

BACKGROUND: The objectives of this study were to assess residents' commitment to caring for the underserved, to determine changes in commitment during residency, and to identify factors associated with sustained commitment.

METHODS: Baseline and follow-up surveys were administered to all internal medicine residents initiating and completing training at Yale-New Haven Hospital from 1996-2002. Commitment to caring for the underserved was assessed with two questions: (1) "Ten years after completing your residency, would you like your clinical practice to have a significant ( $>20 \%$ ) population of patients on public assistance" [yes/no] and (2) Please indicate your level of agreement with the statement "All physicians have an obligation to the medically underserved" [7-point Likert scale]. Residents' baseline and follow-up responses were compared; differences were analyzed with chi-square and Wilcoxon signed-ranks tests.

RESULTS: Paired surveys were available for 137 residents (response rate $=91 \%$ ). For question (1), at baseline, $88(64 \%)$ residents wanted their clinical practice to have $>20 \%$ patients on public assistance. At follow-up, $25 \%$ of residents became less interested and $13 \%$ more interested in caring for patients on public assistance; the overall difference was a $13 \%$ decline. Residents who sustained their commitment to caring for patients on public assistance were more likely to be non-white $(76 \%$ v $56 \% ; \mathrm{p}=0.07)$, desire urban practice $(82 \%$ v $50 \% ; \mathrm{p}<0.01)$, participate in community service during medical school $(68 \% \mathrm{v} 38 \% ; \mathrm{p}=0.02)$, and express interest in international health $(67 \% \mathrm{v} 38 \% ; \mathrm{p}=0.05)$. For question (2), residents' responses regarding an obligation to the underserved fell from a median of moderately agree (at baseline) to agree (at follow-up); this decline was statistically significant $(\mathrm{p}<0.01)$. Over time, residents with substantial financial debt $(>\$ 100,000)$ were more likely to decrease their agreement with this statement $(57 \%$ v $40 \%$; $=0.06)$

CONCLUSION: For this cohort of residents, commitment to caring for the underserved declined significantly over the course of residency. Factors associated with sustained commitment included race, interest in urban practice, and community service participation in medical school. Significant debt appeared to decrease residents' obligation to the underserved. Community service activities in medical school and reduction in debt burden are amenable approaches that may increase commitment to the underserved.

VARIATION IN ACCESS TO CARE BY THE ETHNIC COMPOSITION OF AN INDIVIDUAL'S COUNTY OF RESIDENCE. J.S. Haas ${ }^{1}$, K. Phillips ${ }^{2}$, D. Sonneborn ${ }^{2}$, C. McCulloch ${ }^{2}$, L. Baker ${ }^{3}$, C. Kaplan ${ }^{2}$, E. Perez-Stable ${ }^{2}$, S. Liang ${ }^{2}$; ${ }^{1}$ Brigham and Women's Hospital, Boston, MA; ${ }^{2}$ University of California, San Francisco, San Francisco, CA; ${ }^{3}$ Stanford University, Stanford, CA (Tracking ID \#74881)

BACKGROUND: Ethnic disparities in the use of health care is a persistent problem in the United States. While the majority of studies examining disparities have focused on the characteristics of the individual, more recently there has been growing attention to the notion that an individual's health practices may be influenced by their neighborhood context. We examined whether access to care for individuals of different ethnic groups varies by the ethnic composition of the individual's county of residence.

METHODS: Individuals from the 1996 Medical Expenditure Panel Survey who described their ethnicity as white (W), African-American (AA), or Latino (L), and who resided in one of 451 non-rural counties $(n=14,740)$. Counties were assigned to terciles based on the prevalence of African-Americans and Latinos who resided there. The principal outcome was report of difficulty in obtaining health care. 
RESULTS: In general, African-Americans reported fewer barriers to health care when they lived in a county with a higher prevalence of African-Americans. In addition, AfricanAmericans and whites reported more difficulty obtaining care when they lived in a county with a higher prevalence of Latinos, and Latinos and whites reported more difficulty obtaining care when they lived in a county with a greater prevalence of African-Americans. For example, African-Americans who lived in a county with a high prevalence of Latinos reported more difficulty obtaining care compared to African-Americans who lived in a county with a low prevalence of Latinos $(18.4 \%$ versus $9.5 \%, \mathrm{p}<0.05)$. Conversely, African-Americans who lived in a county with a high prevalence of African-Americans reported less difficulty obtaining care compared to African-Americans who lived in a county with a low prevalence of AfricanAmericans $(9.0 \%$ versus $16.1 \%, \mathrm{p}<0.10)$.

CONCLUSION: AAs may perceive fewer barriers to care when they live in a county with more people of similar ethnicity. AAs, Ls, and Ws may experience more difficulty obtaining care when they live in a county with a higher prevalence of individuals of a different ethnicity. Diminishing disparities in access to health care may require interventions that extend beyond the individual.

EFFECT OF HISPANIC ETHNICITY ON GLYCEMIC TESTING AND CONTROL IN DIABETIC PATIENTS. J.H. Halanych ${ }^{1}$, S.M. Frayne ${ }^{2}$, H. Lin $^{3}$, D. Miller ${ }^{3}$, F. Wang ${ }^{3}$, K.M. Skinner ${ }^{3}$, D.R. Berlowitz ${ }^{1} ;{ }^{1}$ Boston University, Boston, MA; ${ }^{2}$ VA Palo Alto Health Care System, Palo Alto, CA; ${ }^{3}$ Center for Health Quality, Outcomes, and Economics Research, VA Hospital, Bedford, MA (Tracking ID \#74742)

BACKGROUND: Disparities in the quality of diabetes care between African Americans and whites have been extensively documented. Recent small, regional studies highlight differences in care between Hispanics and non-Hispanics. We examined disparities in glycemic monitoring and control, comparing Hispanics to non-Hispanic African Americans and whites in a large national sample of diabetics enrolled in the Veterans Health Administration (VHA). METHODS: Our study sample consisted of veterans enrolled in the VHA in 1998 who were diabetic defined by either 1) receipt of antiglycemic prescriptions or glucose monitoring strips, or 2) at least two diabetes ICD-9 codes in VHA administrative files in 1997-98. Ethnicity was classified as Hispanic, African American, or white based on most recent entry in VHA administrative data. For the subjects with unknown ethnicity, we supplemented with selfreported ethnicity from national VHA survey data (decreasing unknown ethnicity from $19.4 \%$ to $13.5 \%$ ). American Indians, Asians and those of unknown ethnicity were excluded. We compared Hispanics, African Americans, and whites on three dependent variables measured during 1999: receipt of at least one glycosylated hemoglobin (HbA1c) test, mean HbA1c value, and HbAlc value $\geq 9.5$ (guidelines set by the Diabetes Quality Improvement Project). HbAlc testing was identified using CPT procedure code files and/or lab result files. In multivariable analyses all findings were adjusted for age and gender.

RESULTS: Of 446,771 diabetics, $7 \%$ were Hispanic, $16 \%$ African American and $63 \%$ white. Compared to whites, the adjusted odds of receiving annual glycemic testing were 0.83 (95\% CI 0.81-0.86) for Hispanics and 0.90 (0.88-0.92) for African Americans. The mean HbA1c was 7.73 for Hispanics, 7.82 for African Americans, and 7.56 for whites $(\mathrm{p}<0.001)$. The adjusted odds of having very poor glycemic control (HbA1c $\geq 9.5$ ) were $1.25(1.21-1.28)$ for Hispanics and $1.39(1.35-1.41)$ for African Americans compared with whites.

CONCLUSION: In a large, nationally representative sample of diabetics receiving care in an "equal access" health care system, Hispanics received less glycemic testing than both African Americans and whites, and had worse glycemic control than whites. While this study does not distinguish whether patient-level or provider-level factors explain the findings, it supports the recent focus on quality improvement efforts aimed at African Americans, and suggests that similar attention is warranted for Hispanics.

PRIMARY HEALTHCARE FOR WOMEN WITH PHYSICAL DISABILITIES: WHAT ARE WE DOING? J. Hefner ${ }^{1}$; ${ }^{1}$ University of Pittsburgh, Pittsburgh, PA (Tracking ID \#76734)

BACKGROUND: There are nearly 30 million women with disabilities in the United States yet they are among the most underserved. The medical literature shows that a significant proportion of this population feels they have difficulty accessing adequate primary healthcare services. Studies show that this population often lacks opportunities to participate in preventive healthcare activities and does not have access to primary care physicians and facilities. In one study, disabled women perceived more problems with the quality of care rather than the access to care. However, access barriers, including unmet transportation needs, lack of provider knowledge regarding disabilities, refusal of medical treatment, architectural barriers and negative attitudes of providers constitute the majority of the limitations to primary healthcare services.

METHODS: We prospectively collected data on 108 consecutive patients who were seen in the Comprehensive Healthcare Center for Women with Physical Disabilities at MageeWomens Hospital, in Pittsburgh, PA during a three month time period that was randomly selected. This center is a fully handicapped-accessible, hospital-based, outpatient medical office providing primary care and obstetrical and gynecologic services.

RESULTS: Among the 108 patients, only 2 (2\%) stated they did not have a primary care physician (PCP) and $52(48 \%)$ stated they saw their PCP yearly or more. Only 8 patients $(7 \%)$ thought their PCP had discriminated against them. However, in the past 5 years, only six $(6 \%)$ of the 92 non-ambulatory patients had been examined by their PCP on an examining table. Only 42 (51\%) eligible women had a previous screening mammogram. Only $36(33 \%)$ patients had a screening Pap smear in the past 5 years. Ten (9\%) patients were on therapy for osteoporosis and $20(19 \%)$ said they either had a DEXA scan previously or had it recommended by their PCP. The age range evaluated by DEXA scanning was 33-68 years. Lumbar spine DEXA scan T-Scores reveal that 58 patients (59\%) had either osteoporosis or osteopenia (Range -1.5 to -5.2$)$. Hip DEXA scan T-Scores reveal that $80 \%(82 \%)$ had either osteoporosis or osteopenia (Range -1.5 to -6.8 ).

CONCLUSION: Women with physical disabilities represent a unique, underserved population. Our data suggests that while patients identify themselves as having a PCP, few are actually receiving adequate preventive healthcare services. While transportation and access constitute the major barrier to preventive healthcare services, we speculate that lack of physician knowledge and training in the primary care of the physically disabled woman, may lead to undesirable and unnecessary negative outcomes such as hip fractures. Primary healthcare is the responsibility of the PCP. Future research should focus on raising the awareness of the inadequate primary care that exists for this population.

THE HEALTH EFFECTS OF RESTRICTING PRESCRIPTION MEDICATION USE DUE TO COST. M. Heisler ${ }^{1}$, K.M. Langa ${ }^{1}$, E.L. Eby ${ }^{2}$, A.M. Fendrick ${ }^{1}$, M. Kabeto ${ }^{1}$, J.D. Piette ${ }^{1}$. ${ }^{1}$ University of Michigan, Ann Arbor, MI; ${ }^{2}$ VA HSR\&D Field Office, Ann Arbor, MI (Tracking ID \#74173)

BACKGROUND: High out-of-pocket expenditures for prescription medications may lead people with chronic illnesses to restrict their use of these medications. Whether adults experience adverse health outcomes after having restricted medication use because of cost is not known.

METHODS: We performed a prospective cohort study using two waves of the Health and Retirement Study (HRS), a national survey of adults aged 51 to 61 in 1992, and the Asset and Health Dynamics Among the Oldest Old (AHEAD) Study, a national survey of adults aged 70 or older in 1993. We used multivariable logistic and linear regression models to assess the independent effect on health outcomes over 2-3 years of follow-up of reporting in 1995/96 having taken less medicine than prescribed because of cost during the prior two years. After adjusting for differences in sociodemographic characteristics, health status, and comorbid chronic conditions, we determined the risk of a significant decline in overall health among respondents in good to excellent health at baseline and of developing new disease-related adverse outcomes among respondents with cardiovascular disease, diabetes, arthritis, and depression. RESULTS: In adjusted analyses, $29.9 \%$ of those who had restricted medications because of cost reported a significant decline in their health status, compared to $20.3 \%$ of those who had not (AOR: 1.80, CI: 1.28-2.53). Respondents with cardiovascular disease who restricted medications reported higher rates of angina ( $15.7 \%$ vs. $11.4 \%$, AOR: $1.49 \mathrm{CI}: 1.06-2.10)$ and experienced higher rates of non-fatal heart attacks or strokes $(10.9 \%$ vs. $7.4 \%, \mathrm{AOR}: 1.55, \mathrm{CI}$ 1.06-2.27). After adjusting for potential confounders, we found no differences in diseasespecific complications among respondents with arthritis, diabetes, and depression. Patterns of adverse outcomes among restrictors were similar in the two different age cohorts.

CONCLUSION: Both middle-aged and elderly Americans who restrict prescription medications because of cost face an increased risk of adverse health outcomes from this restriction. Such cost-related medication restriction may be an important mechanism for worse health outcomes among low-income and other vulnerable populations who lack adequate insurance coverage.

WARNING: EXPOSURE TO VIOLENCE MAY BE HAZARDOUS TO YOUR HEALTH. A.E. Hendifar ${ }^{1}$, W. Fisher ${ }^{2}$, K.B. DeSalvo ${ }^{1}$; ${ }^{1}$ Tulane University, New Orleans, LA; ${ }^{2}$ MetaMetrics, Inc., Durham, NC (Tracking ID \#75489)

BACKGROUND: The psychological impact of exposure to violence (ETV) on children is documented. However, its impact on physical health, chronic disease expression, and health behaviors of adults has not been explored. We investigated the relationship between reported ETV and health related measures in a low-income, inner city population.

METHODS: 135 adult patients presenting for routine primary care appointments participated in a survey. Subjects were read an instrument that queried medical history, family medical history, social and physical functioning, health attitudes and behaviors, and violence exposure. No patients declined to participate.

RESULTS: Respondents were predominately black (86\%) and female (75\%). $77 \%$ were middle-aged, $56 \%$ had earned a high school diploma and $56 \%$ had annual incomes less than $\$ 9000.72 \%$ of the patient population responded positively to one of the three measures of ETV. ETV data was fitted in Rasch item response model for rating scales. Rasch analysis showed the statement: "I have family members or friends that have been murdered" had high reliability $(0.98)$ and had the highest item internal consistency. It demonstrated the best properties of a screening question and was selected as our measure of ETV. 55\% agreed to the statement. In bivariate analyses, those exposed to violence were more likely to be black ( $\mathrm{p}=$ $0.03)$, smoke cigarettes $(\mathrm{p}=0.03)$, visit the emergency department $(\mathrm{p}<0.01)$, have guns in the home $(\mathrm{p}=0.01)$, and carry guns $(\mathrm{p}=0.01)$. Those exposed to violence were more likely to report family members with alcohol addiction $(\mathrm{p}=0.05)$, cancer $(\mathrm{p}<0.01)$ heart disease $(\mathrm{p}<0.01)$, stroke $(\mathrm{p}=0.03)$, and asthma $(\mathrm{p}=0.02)$. Those with violence exposure had clinically worse physiologic variables for SBP (143 vs. 140$)$, DBP ( 80 vs. 78$)$, HgbA1C ( 8.5 vs. 7.0$)$, total cholesterol (221 vs. 211$)$ and body mass index (32 vs. 31$)$. In multiple regression analyses adjusting for age, race, gender and income, exposure to violence was associated with visits to the emergency department (OR $3.2[1.5,6.6])$, smoking (OR $3.0[1.2,7.2])$, a family history of cancer (OR 3.6 [1.8, 7.8]), a family history of heart disease (OR $3.8[1.8,7.9])$, and having guns in the home (OR $3.4[1.1,11.1])$.

CONCLUSION: ETV is associated with worse objective and subjective reported personal and family health, higher emergency department use, and owning and carrying firearms in lowincome adults living in an urban center. Based on these preliminary data, a further study investigating the relationship between ETV and key health measures is warranted.

ARE BETA BLOCKERS UNDER-UTILIZED AS HYPERTENSION THERAPY? A. Huebschmann ${ }^{1}$, C. Bublitz ${ }^{1}$, R.J. Anderson ${ }^{1} ;{ }^{1}$ University of Colorado Health Sciences Center, Denver, CO (Tracking ID \#75167)

BACKGROUND: The most recent Joint National Committee (JNC) reports have recommended the use of diuretics or beta-blockers as first-line therapy for hypertensive patients without other comorbidity. We wanted to look at patterns of usage of antihypertensive agents with respect to this guideline.

METHODS: Data were obtained from the 1995-2000 National Ambulatory Medical Care Surveys (NAMCS). Chi-squared tests of association were performed to determine if the usage of 
Beta Blockers (BB), Angiotensin-converting enzyme inhibitors (ACEI), diuretics and calciumchannel blockers (CCB) by NAMCS essential hypertension patients changed between 1995 2000. All estimates reported in this analysis are reliable estimates (i.e. relative standard error \{RSE\} is less than $30 \%$ ). Logistic regression analysis determined associations between medication use and NAMCS year (1995-2000) while controlling for gender, race (white, non-white), region, urbanicity and geriatric age ( $<65$ years of age, 65 years of age or older). SAS callable SUDAAN software was used for all statistical procedures. Data were weighted for the analysis.

RESULTS: By Chi-squared analysis, there was a statistically significant trend of increased BB usage over the years 1995-2000 in the 332,510,280 essential hypertension visits examined with prevalence rising from $16.42 \%$ in 1995 to $21.86 \%$ in $2000, p=0.0337$. Using logistic regression analysis, the adjusted odds ratio per NAMCS year was $1.08,\{95 \%$ CI $1.04,1.13\}$, $\mathrm{p}=0.0004)$. There was no trend towards increased or decreased use in the other antihypertensive groups ( $\mathrm{p}>0.05$ ), as ACEI use ranged from $23.9-29.4 \%$, diuretics varied from $25.8-30 \%$, and CCBs were used in $23.0-29.7 \%$ over this time frame.

Interestingly, non-white hypertensive patients were less likely to receive beta blockers than white patients (OR: $0.76,95 \% \mathrm{CI}\{0.63,0.91\}, \mathrm{p}=0.0042$ ). Only CCBs were also prescribed differently based on race with non-white hypertensives being more likely to receive these medications (OR: 1.46, $95 \%$ CI $\{1.24,1.73\}, \mathrm{p}<0.0001)$.

CONCLUSION: Diuretics, ACEIs, and CCBs were the most commonly used antihypertensive agents over the timeframe studied as approximately $25 \%$ of all patients were on each of these medications while BBs only reached a $21.8 \%$ share in 2000 . There was a significant trend of increased BB usage over this time frame. However, BB therapy appears to be under-utilized in the non-white hypertensive population.

HOMELESS PEOPLE'S INTERACTIONS WITH AND TRUST IN PARAMEDICS AND POLICE IN TORONTO. S.W. Hwang ${ }^{1}$, T.L. Zachrison ${ }^{2}$, P.A. Hamel ${ }^{2}$; ${ }^{1}$ St. Michael's Hospital, Toronto, Ontario; ${ }^{2}$ University of Toronto, Toronto, Ontario (Tracking ID \#76603)

BACKGROUND: Homeless people's previous interactions with and level of trust in paramedics and police officers may affect their willingness to seek emergency assistance. Of particular concern, news reports have documented incidents in which police officers used excessive and unjustified force against homeless people. The objectives of this study were to assess homeless people's interactions with and trust in paramedics and police in Toronto, Ontario.

METHODS: We interviewed a systematic sample of 160 homeless persons at 18 of the largest drop-in centers and shelters for single adults and youth in Toronto in 2001. Interviewers approached every 10th person in sleeping quarters and common areas. Frankly psychotic and severely intoxicated individuals were excluded. Subjects were told that the study examined their "perceptions of emergency services." After giving informed consent, subjects were asked about demographics, number of interactions with paramedics in Toronto in the past 12 months, whether they considered any of these interactions "good" or "bad," detailed descriptions of these interactions, and rating of their trust in paramedics on a 0 -to- 5 Likert scale. Identical questions were then asked regarding police. Finally, subjects were asked if they had been assaulted (defined as a "sudden, violent physical attack") during the past 12 months, and if so, by whom. Differences in responses regarding paramedics and police were examined using the binomial test or the Wilcoxon signed ranks test.

RESULTS: Subjects had the following characteristics: mean age, 36 years; male $76 \%$; white $55 \%$, black $23 \%$, other race $22 \%$; duration of current episode of homelessness $<6$ months $45 \%$, $6-12$ months $28 \%,>12$ months $25 \%$. Interaction in the past 12 months was more common with police than with paramedics $(61 \%$ vs. $37 \%, \mathrm{p}=0.0001)$, as was the occurrence of at least one interaction described as "bad" ( $39 \%$ vs. $9 \%, \mathrm{p}=0.0001)$. Trust levels were significantly lower in police compared to paramedics (median 3 vs. $5, \mathrm{p}=0.0001$ ). The proportion of subjects who reported experiencing an assault in the last 12 months by anyone, by a police officer, and by a paramedic was $36 \%, 9 \%$, and $0 \%$, respectively. The $95 \% \mathrm{CI}$ around these estimates was $\pm 5 \%$.

CONCLUSION: Homeless people in Toronto express much lower levels of trust in police than in paramedics. The frequency of reported experiences of assault by police is concerning. Additional studies are needed to confirm these findings and to objectively characterize interactions between homeless people and police officers.

IS PERCEIVED RACIAL DISCRIMINATION ASSOCIATED WITH DISPARITIES IN BREAST AND CERVICAL CANCER SCREENING? E. Jacobs ${ }^{1}$, K. Karavolos ${ }^{2}$, P.J. Rathouz ${ }^{3}$, L. Powell ${ }^{2} ;{ }^{1}$ Cook County Hospital, Chicago, IL; ${ }^{2}$ Rush Medical College, Chicago, IL; ${ }^{3}$ University of Chicago, Chicago, IL (Tracking ID \#76595)

BACKGROUND: Disparities in breast and cervical cancer screening between whites and other racial/ethnic groups have been well documented. These disparities are only partially explained by socioeconomic status and access to health care services. The objective of this study was to determine if perceived racial discrimination is also associated with lower rates of breast and cervical cancer screening in a longitudinal study.

METHODS: We conducted a 2-year prospective cohort study of 3717 women between the ages of 42 and 52 participating in the Study of Women's Health Across the Nation in one of seven large U.S. cities. We excluded women who had been diagnosed with breast or cervical cancer or who had had a hysterectomy. Data for this study were collected yearly from each woman via interview and self-report questionnaire. We used generalized estimating equation logistic regression models to understand the relationship between reported receipt of mammograms, breast exams, and Pap smears to racial discrimination while adjusting for ethnicity (Asian, black, Hispanic \& white), income, education, insurance status, English language proficiency, and use and access to health care.

RESULTS: Nine hundred and forty women in the cohort reported racial discrimination in year 1. Perceived racial discrimination was significantly associated with lower receipt of mammo- graphy $(\mathrm{z}=3.03, \mathrm{p}<0.01)$, breast examination $(\mathrm{z}=2.77, \mathrm{p}=0.01)$ and Pap smears $(\mathrm{z}=2.05$, $\mathrm{p}=0.04)$, translating into adjusted odds ratios of $0.81(95 \% \mathrm{CI}=0.71-0.93)$ for receiving a mammogram, $0.81(95 \% \mathrm{CI}=0.69-0.94)$ for receiving a breast exam, and $0.86(95 \% \mathrm{CI}=$ $0.71-0.93)$ for receiving a Pap smear if a woman reported experiencing racial discrimination compared to no discrimination. Other variables significantly associated with receiving each of the three exams were income, type of health insurance, and ethnicity. Education and language were also significantly associated with receipt of breast exams and pap smears, but not mammography. Including use of and access to health care in the models did not significantly change the results.

CONCLUSION: Self-report of racial discrimination was associated with a significant reduction in the odds of receiving breast cancer and cervical cancer screening. This study suggests that discrimination is one of several important factors determining breast cancer screening and should be targeted in efforts to reduce racial disparities in prevention screening.

DIFFERENCES IN MEDICAL CARE AND DISEASE OUTCOMES AMONG BLACK AND WHITE WOMEN WITH HEART DISEASE. A.K. Jha ${ }^{1}$, P.D. Varosy ${ }^{2}$, A. Kanaya ${ }^{2}$ D.B. Hunninghake ${ }^{3}$, M.A. Hlatky ${ }^{4}$, D. Waters ${ }^{2}$, C.D. Furberg ${ }^{5}$, M. Shlipak ${ }^{2} ;{ }^{1}$ Brigham and Women's Hospital, Boston, MA; ${ }^{2}$ University of California, San Francisco, San Francisco, CA; ${ }^{3}$ University of Minnesota, Minneapolis, MN; ${ }^{4}$ Stanford University, Stanford, CA; ${ }^{5}$ Wake Forest University, Winston-Salem, NC (Tracking ID \#73607)

BACKGROUND: The risk of cardiovascular mortality is higher among blacks than among whites and this disparity is more pronounced in women for unknown reasons. We sought to evaluate differences in medical care and outcomes among black and white women with heart disease.

METHODS: Among the 2,699 black and white women enrolled in the Heart and Estrogen/ progestin Replacement Study (HERS), we used Cox proportional hazards models to determine whether race was associated with risk of coronary heart disease (CHD) events (cardiovascular death or non-fatal myocardial infarction). Further, we evaluated whether there were racial differences in the use of appropriate medical therapies, and adequate control of systolic blood pressure, glucose, and LDL cholesterol levels.

RESULTS: During an average of 4.1 years of follow-up, CHD events were twice as likely in black compared with white women (6.4 per 100 person-years versus 3.1 per 100 person-years, hazard ratio, 2.1; $95 \%$ confidence interval, 1.5 to $2.8 ; \mathrm{p}<0.001$ ). Black women had higher baseline rates of hypertension, diabetes, and hypercholesterolemia, yet were less likely to report taking aspirin or statins. Rates of beta-blocker use among women with prior myocardial infarctions, and ACE-inhibitors among women with congestive heart failure were similar in the two groups. Black women were less likely to have optimal baseline blood pressure ( $56 \%$ versus $63 \%, \mathrm{p}=0.01)$ or LDL cholesterol $(30 \%$ versus $38 \%, \mathrm{p}=0.04)$ control than white women and these differences persisted during follow-up. After adjusting for these and other baseline differences, black women still had a nearly $50 \%$ higher risk of CHD events (multivariableadjusted hazard ratio, $1.5 ; 95 \%$ confidence interval, 1.1 to $2.1 ; \mathrm{p}=0.02$ ).

CONCLUSION: In a large study of women with heart disease, black women were less likely to be taking appropriate preventive therapy and less likely to have adequate risk factor control despite a nearly $50 \%$ greater CHD event risk compared with white women. Interventions to increase use of appropriate therapy and improve risk factor control in black women are needed

GENDER DIFFERENCES IN OUTPATIENT QUALITY OF CARE IN THE VETERANS AFFAIRS HEALTHCARE SYSTEM. A. Jha ${ }^{1}$, J.B. Perlin ${ }^{2}$, M. Steinman ${ }^{3}$, J.W. Peabody ${ }^{3}$, J.Z. Ayanian ${ }^{4}$; ${ }^{1}$ Brigham and Women's Hospital, Boston, MA; ${ }^{2}$ Veterans Health Administration, Washington, DC; ${ }^{3}$ University of California, San Francisco, San Francisco, CA; ${ }^{4}$ Brigham and Women's Hospital, Dept of Health Care Policy, Harvard Medical School, Boston, MA (Tracking ID \#73693)

BACKGROUND: Women may receive lower quality inpatient care than men. Because quality of outpatient care by gender is largely unexplored, we examined whether gender differences exist in the VA healthcare system, in which women are a rapidly growing population and currently comprise $10 \%$ of the 4 million enrollees.

METHODS: We used data from the External Peer Review Program, a cross-sectional sample of patients with common conditions and $>1$ primary care visit at a VA medical center between 10/1999 and 9/2000, with over-sampling of women. Using logistic regression with generalized estimating equations, we assessed the associations between gender and 7 common disease- or age-specific quality-of-care measures, adjusting for age, hospital characteristics, and clustering by regional VA network.

\begin{tabular}{|l|c|c|c|c|}
\hline Quality Indicator & Women \% & Men \% & $\begin{array}{c}\text { Adjusted RR } \\
(95 \% \text { CI })\end{array}$ & P \\
\hline Diabetic eye screen & 67.0 & 68.6 & $0.98(0.96,100)$ & 0.10 \\
\hline Amnual HgBA1c & 94.7 & 94.3 & $100(0.99,101)$ & 0.71 \\
\hline HpBA1c $\leq 8.0$ mg/dI. & 61.1 & 62.0 & $105(103,107)$ & $<0.01$ \\
\hline Blood pressure $\leq 140 / 90$ & 48.0 & 47.4 & $0.96(0.93,0.99)$ & 0.02 \\
\hline Pneumococcal vaccinalion & 74.0 & 79.7 & $0.98(0.97,0.99)$ & $<0.01$ \\
\hline Influenza vaccination & 73.5 & 76.5 & $0.99(0.97,100)$ & 0.03 \\
\hline Colon cancer screening & 68.9 & 699 & $0.99(0.97,100)$ & 0.10 \\
\hline
\end{tabular}

RESULTS: Among 86,342 patients from nearly every VA hospital and clinic in the United States, women represented $13 \%$ to $23 \%$ of the sample for each quality measure. Adherence to indicators for women and men ranged from $47 \%$ for blood pressure control to over $90 \%$ for $\mathrm{Hgb}$ A1c testing. Slightly lower vaccination rates for women were partly explained by their younger age. Adjusted relative risks (converted from odds ratios) for women relative to men ranged from 0.96 to 1.05 across the 7 quality measures: 
CONCLUSION: For common outpatient measures, women and men received remarkably similar quality of care in the VA healthcare system, suggesting quality improvement efforts in VA outpatient care have been implemented consistently for women and men.

HOW DO PATIENTS' PERCEPTIONS OF MEDICAL CARE RELATE TO THEORETICAL CONSTRUCTS OF CULTURAL COMPETENCE? R.L. Johnson ${ }^{1}$, S. Saha ${ }^{2}$, J.J. Arbalaez ${ }^{1}$, M.C. Beach ${ }^{1}$, L.A. Cooper ${ }^{1}$; ${ }^{1}$ Johns Hopkins University, Baltimore, MD; ${ }^{2}$ Oregon Health Sciences University, Portland, OR (Tracking ID \#76382)

BACKGROUND: As cultural competence gains increasing attention among practicing physicians and medical educators, there is a need to move beyond theory and anecdotes to better understand how culture impacts patient care. The goal of this study was to explore whether differences exist across racial and ethnic groups in respondents' perceptions of the degree of cultural competence demonstrated by their individual physician(s) (MD) and the health care system within which they seek medical care.

METHODS: Data from the Commonwealth Fund's 2001 Health Care Quality Survey was analyzed. This national telephone survey of 6,722 adults living in the United States was collected using random-digit-dialing methods; over-sampled respondents from communities with high concentrations of African-American, Latino and Asian households; and yielded an overall response rate of $54.3 \%$. Survey weighted descriptive statistics and logistic regression analyses were performed. Five outcome measures are used to evaluate patients' perceptions of cultural competency. The first four address: 1) respondents' trust in their MD, 2) MD treatment of respondents with respect and dignity, 3) MD understanding of respondents' cultures and values, and 4) MD disapproval or looking down on respondents' lifestyles. The fifth addresses cultural competence at the health care delivery system level and asks whether respondents believe they would receive better health care if they were of a different race/ ethnicity than they actually are.

RESULTS: Responses to questions about MD and health care system cultural competence experiences were statistically significantly different $(\mathrm{p}<0.05)$ for one or more minority groups even when controlling for SES, age, health status, source of care, MD-patient communication, and health literacy. Asians were less likely $(\mathrm{p}<0.05)$ than whites to report that their doctor treats them with a great deal of respect $(\mathrm{OR}=0.66)$ and were more likely $(\mathrm{p}<0.001)$ than whites to report that their doctor looks down on them or the way they live their live $(\mathrm{OR}=$ 2.41). African Americans, Hispanics and Asians were more likely $(\mathrm{p}<0.001)$ than whites to say that they would have received better medical care if they were of a different race/ethnicity $(\mathrm{OR}=12.13, \mathrm{OR}=7.81$, and $\mathrm{OR}=8.03$ respectively $)$.

CONCLUSION: This research suggests the need to move away from theoretical and anecdotal discourse about cultural competence towards empirical study with nationally representative samples of racial/ethnic minorities. Our results advocate for broadening the cultural competence discourse beyond understanding culture-specific practices and perceptions to closer examination of what biases are inherent in the U.S. medical system and how they affect racial/ethnic minorities' perceptions of care.

PATIENTS CHOOSING A PRIVATE PRACTICE OR A UNIVERSITY OUTPATIENT CLINIC FOR THEIR FOLLOW-UP: ARE THEY THE SAME? $\underline{\text { N. Junod Perron }}{ }^{1}$, B. Favrat ${ }^{2}$ P. Vaucher ${ }^{2}$, A. Pecoud ${ }^{2}$, M. Vannotti ${ }^{2} ;{ }^{1}$ Policlinique de médecine, Geneva, Switzerland; ${ }^{2}$ Policlinique Médicale Universitaire, Lausanne, Switzerland (Tracking ID \#73865)

BACKGROUND: In many countries, patients have the opportunity to attend University outpatient clinics providing community care as well as training for medical residents where physicians change regularly and interpersonal continuity of care is not provided. The aim of the study was to evaluate the differences between patients attending a University outpatient clinic and patients consulting a private practice and explore the reasons for choosing a practice and remaining attached to it.

METHODS: We conducted a 4-month cross-sectional study in the University outpatien clinic of Lausanne, Switzerland and ten private general practices located in the neighborhood and randomly selected. Eligible subjects were $>30$ years old patients, Swiss national or long term residents, suffering from one or more chronic conditions and attending the same practice for $>3$ years. They were given a questionnaire about socio demographic data, use of medical resources and reasons for choosing and remaining at the same practice. We conducted then in-depth interviews with 26 patients selected from the first part of the study. They were audiotaped, transcribed ad verbatim and examined using basic content analysis.

RESULTS: 329 patient questionnaires were obtained and analyzed, 219 from private practice and 110 from outpatient clinic patients. More patients attending the outpatient clinic were male $(58.7 \%$ vs $33.8 \%, \mathrm{p}<0.01$ ), with lower education (schooling $<10$ years: $44.1 \%$ vs $30.8 \%$, $\mathrm{p}<0.01$ ) and lower income ( $<3500 \mathrm{SF}: 64.6 \%$ vs. $34.2 \%, \mathrm{p}<0.01)$. Private practices patients used the emergency settings less often (OR 0.5 CI 95\%: 0.3-0.9). Main reasons for choosing a private practice versus an outpatient clinic were recommendation (OR 4.4 CI 95\% 1.9-9.9) and the fact that relatives or acquaintance were already registered (OR 5 CI 95\%: 2.1-11.9) and not because it was the only practice known (OR 0.09 CI 95\%; 0.01-0.5) or because they could quickly obtain a first appointment (OR 0.33 CI 95\%: $0.15-0.7$ ). Unlike outpatient clinic patients, private practice patients attached more importance to physician communication skills (OR 4.81 CI 95\%: 2.2-10.5), home calls (OR 5.56 CI95\%; 1.7-18.7), trust (OR 2.33 CI95\%: $1.1-5$ ) than to investigations facilities (OR 0.38 CI 95\%: $0.15-0.8$ ). Analysis of the 26 interviews (13 from private practice patients and 13 from outpatient clinic patients) revealed that more patients attended private practices for psycho-social problems while more patients consulted the outpatient clinic for urgent somatic problems because of convenient hours or investigations facilities. Although outpatient clinic patients reported more biomedical expectations at the first consultation, when asked about general expectations, they indicated similar biomedical and communication expectations but payed less attention to the relationship aspects of care. Many outpatient clinic patients considered the change of physician to be positive because physicians showed more enthousiasm and professional interest. They also appreciated team work.
CONCLUSION: Long-term patients attending a University outpatient clinic seem to be more vulnerable and use more the medical resources than those consulting in private practices. Although outpatient clinic patients pay more attention to investigation facilities than to communication skills, the qualitative analysis reveals that general expectations and perception of the relationship are not so different among both groups and that reasons and circumstances to seek care are complex.

UNDERSTANDING WHAT THE DOCTOR SAID: ABNORMAL MAMMOGRAMS IN ETHNICALLY DIVERSE PATIENTS. L.S. Karliner ${ }^{1}$, C.P. Kaplan ${ }^{1}$, T.C. Juarbe ${ }^{1}$ G. Gildengorin ${ }^{1}$, E.J. Perez-Stable ${ }^{1}$; ${ }^{1}$ University of California, San Francisco, San Francisco, CA (Tracking ID \#75967)

BACKGROUND: Screening mammography every 2 years for women 50 to 69 years of age may lead to $50 \%$ having an abnormal study every 10 years. We explored the role of ethnicity and English proficiency in fully understanding explanation of abnormal mammography results.

METHODS: Women with confirmed abnormal mammograms were recruited from 5 clinical sites to participate in a survey focused on communication and quality of life concerns. Women were eligible if between age 40 and 80 years, had no previous history of breast cancer, self-identified as White, African American (AA), Latina or Asian/Pacific Islander (API) and spoke English, Spanish or Chinese. The questionnaire included demographics, items on language background, level of comprehension of the mammogram results, ethnicity and language use with physician and preference to have doctors of their same ethnic background.

RESULTS: 970 women were interviewed. The sample included 239 AAs, 143 APIs (primarily of Chinese descent), 182 Latinas and 404 Whites. Mean age was 56.1 years and mean years of education was 13.8. Most interviews were completed within 4 months of the index mammography and $31 \%$ had a suspicious or highly suspicious result; $15 \%$ had an indeterminate examination. Overall, $70 \%$ of women reported that they understood fully their doctors' explanation of their abnormal mammogram, but this differed by ethnicity. AA (72\%) and White $(73 \%)$ women were more likely than API $(63 \%)$ and Latina $(66 \%)$ women to report that they understood fully their doctors' explanation $(\mathrm{p}=.09)$. To explore possible reasons for this difference, we examined the relationships between language proficiency and education, and women's reported level of understanding. Fewer Latinas than API women reported speaking only English with their doctors (54\% versus 70\%). Latina and API women who spoke at least some English with their doctors were more likely than those who spoke only Spanish or Chinese to report that they understood fully their doctor's explanation (68\% vs. $59 \%$ for limited English proficiency speakers, $\mathrm{p}=.12$ ). However, we found no difference in mean number of years in school between those Latina (10.7 vs. 10.2 years) and API (13.6 vs. 14.1 years) women who reported they understood fully their doctor's explanation and those who did not.

CONCLUSION: Although a majority of women do understand their doctors' explanation of their abnormal mammograms, API and Latina women were less likely to have a full understanding, and those with limited English proficiency had the least full understanding.

CHRONIC HOMELESSNESS, ADDICTION AND HEALTH RELATED QUALITY OF LIFE. S.G. Kertesz ${ }^{1}$, N.J. Horton ${ }^{2}$, M.J. Larson ${ }^{3}$, J.H. Samet ${ }^{2}$; ${ }^{1}$ U. Alabama Birmingham, Birmingham, AL; ${ }^{2}$ Boston U., Boston, MA; ${ }^{3} \mathrm{New}$ England Research Institutes, Watertown, MA (Tracking ID \#74198)

BACKGROUND: Persons fulfilling a new federal definition of "chronically homeless" have been proposed for special assistance, but the distinct character of this group has received limited study. Using prospective cohort data, we tested the hypothesis that chronically homeless persons have uniquely poor health-related quality of life (HRQOL) over time, and tested whether substance use could account for observed differences.

METHODS: Subjects $(N=470)$ were recruited at the time of detoxification for alcohol or drugs, and followed for 2 years. The primary independent variable was residential status. Subjects available for 3 assessments $(N=289)$ were classified as Chronically Homeless $(\mathrm{CH}$, $\mathrm{N}=64$ ), Residentially Stable (RS, $\mathrm{N}=110$ ), and Transitional ( $T R, N=115$ ) based on reported nights homeless. At each follow-up we measured HRQOL with the SF-36 Mental Component Score (MCS) and Physical Component Score (PCS). We tested whether residential status was associated with MCS and PCS by fitting general linear regression models for correlated data that account for multiple observations per subject, controlling for age, sex, race, time \& the interaction of residential status \& time.

RESULTS: At baseline, CH subjects had very low MCS scores (mean 30.7), as did RS (32.2) and TR subjects (30.6), values lower than scores found in depression. While RS MCS scores improved 13.5 points, $\mathrm{CH}$ showed less improvement $(+5.6, \mathrm{p}=.01$ for Time by Group interaction). Adjustment for ongoing substance use did not explain the impact of chronic homelessness on MCS. The Figure shows predicted mean SF-36 MCS scores over time for the 3 groups. A model for PCS showed similar improvement over time for all residential groups. CONCLUSION: The chronically homeless had poor mental HRQOL upon detoxification entry, and much less improvement than others over 2 years, a finding not explained by continuing substance use. Our study identifies a unique health vulnerability among the chronically homeless.

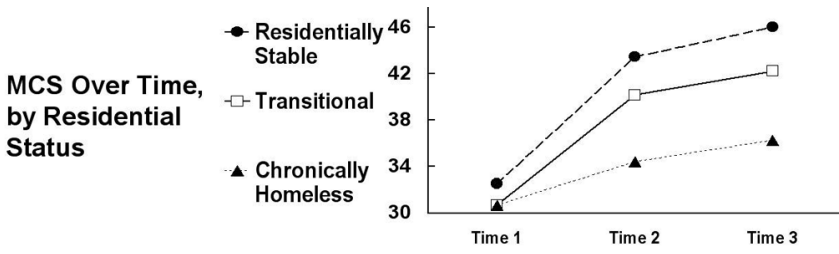


DOES HOSPITAL DISCHARGE TO A HOMELESS MEDICAL RESPITE UNIT AVERT READMISSION? S.G. Kertesz ${ }^{1}$, S. Swain ${ }^{2}$, M. Posner ${ }^{3}$, M. Shwartz ${ }^{3}$, J. O'Connell ${ }^{2}$, A.S. Ash ${ }^{3} ;{ }^{1} U$. Alabama at Birmingham, Birmingham, AL; ${ }^{2}$ Boston Health Care for the Homeless Program, Boston, MA; ${ }^{3}$ Boston U., Boston, MA (Tracking ID \#74277)

BACKGROUND: A novel subacute care facility, the medical respite unit, offers homeless patients recuperative care while addressing psychosocial and other homeless-specific needs. Such units exist nation-wide, but their effectiveness is unstudied and they are unrecognized by the Centers for Medicare and Medicaid Services. We used data from the largest such program in the U.S. to test whether discharge to a medical respite was associated with delayed hospital readmission or death (R/D) for the hospitalized homeless.

METHODS: We examined time to first R/D for 784 homeless adults discharged from a medical/surgical hospitalization 7/98-6/01, with follow-up to 6/02. The predictor of interest was discharge destination, in 4 categories: Respite, Own Care (shelters/streets), Other Care (e.g. nursing home), and Left Against Medical Advice (AMA). We used survival curves and sequential proportional hazards models to quantify the effect of discharge destination on the hazard for R/D. We examined raw differences, and controlled for factors predicting R/D, i.e. comorbidity using Diagnostic Cost Groups, index hospitalization length of stay (LOS), number of prior hospitalizations, substance abuse, \& sociodemographics. Data came from crosslinking 7 administrative sources.

RESULTS: Raw survival curves for the combined outcome of R/D did not differ by discharge destination ( $\mathrm{p}=.71)$. Compared to Own Care patients, Respite patients had features associated with increased hazard for R/D, including greater comorbidity, and LOS (all $\mathrm{p}<.01$ ). The Table shows unadjusted \& adjusted results for R/D:

\begin{tabular}{lcccc} 
& Respite & Own Care & Other Care & AMA \\
N (Patients) & 136 & 433 & 174 & 41 \\
\hline \% with R/D at 6 months & $18 \%$ & $21 \%$ & $23 \%$ & $22 \%$ \\
Unadjusted Hazard for R/D & $1.0(.78,1.30)$ & REF & $1.10(.87,1.40)$ & $1.22(.81,1.85)$ \\
Adjusted Hazard for R/D & $0.86(.65,1.13)$ & REF & $0.87(.68,1.11)$ & $1.12(.73,1.74)$
\end{tabular}

CONCLUSION: At discharge, respite patients had higher risk for readmission or death compared to those discharged to Own Care, yet they experienced similar readmission and death rates. Risk adjustment suggested a protective effect of Respite (adjusted hazard $=0.86$ $\mathrm{p}=0.26$ ). Our findings, though limited by sample size and possibly residual confounding, support continued interest in medical respite for homeless persons.

EXPECTATIONS AND APPROPRIATE USE OF A RURAL EMERGENCY DEPARTMENT. A.A. Khan ${ }^{1}$, K. Kroenke ${ }^{2}$, R.M. Lubitz ${ }^{3} ;{ }^{1}$ Indiana University, Indianapolis, IN; ${ }^{2}$ Regenstrief Institute, Indianapolis, IN; ${ }^{3}$ St. Vincent Hospital, Carmel, IN (Tracking ID \#75943)

BACKGROUND: Patients who utilize rural non-teaching hospitals, and particularly their emergency departments (ED), may have different healthcare needs and expectations than other well-studied settings. Because there is little research in this area, we assessed patient expectations of care and appropriateness of utilization of a rural ED.

METHODS: We prospectively recruited 200 random patients from a single moderate sized rural ED (annual visits = about 9500). Subjects completed a questionnaire assessing the reason for visit expectations of the ED and physician. They also completed the Patient Health Questionnaire, a well-validated measure of depression, anxiety and somatization. Sociodemographic data and clinical information were obtained by reviewing ED encounter records. ED physicians completed a short questionnaire assessing nature of the visit, severity and appropriateness.

RESULTS: The study sample was $61 \%$ female with a mean age of 31 years (infant- 92 ); $74 \%$ were $>18$ years old; $90 \%$ were local residents and $81 \%$ had a primary care doctor; $52 \%$ were employed and $72 \%$ had health insurance. Most $(61 \%)$ had symptoms of $<24$ hours and $62 \%$ considered them emergent. Expectations of treatment varied: patients expected explanation of their condition $(36 \%)$, prescription of medicines $(32 \%)$, a test in the ED $(25 \%)$ or a medication or injection (24\%); $31 \%$ expected immediate (few hours) relief from symptoms. ED physicians considered $44 \%$ of patents to have unreasonable expectations, $55 \%$ of visits inappropriate and $38 \%$ of visits avoidable if the patient had prompt access to their own doctor. Overall severity of illness was low (mean $=3$ out of 9). ED physicians disagreed with patients about the nature of emergency, particularly in terms of perception of severity (kappa: $0.164, p=0.005$ ) and appropriateness of the visit (kappa: 0.132, p $=0.061$ ). Recognition of a non-physical etiology was important as it independently predicted (by multiple regression analysis) inappropriate visit (OR: 2.3, $\mathrm{p}=<0.001$ ) and frequent ED user status (OR: $1.5, \mathrm{p}=0.047)$. Inappropriate visit was also predicted by longer duration of symptoms (OR: 1.7, p =<0.001), frequent ED user status (OR: 1.9, $\mathrm{p}=0.004)$ and depression (OR: $2.0, \mathrm{p}=0.047$ ).

CONCLUSION: In this rural ED, both patient and physician reports identified unreasonable expectations and inappropriate ED use. Findings suggest opportunities for patient and system interventions to increase appropriate use and patient/provider satisfaction.

AN EPIDEMIC OF DIABETES MELLITUS IN AN URGENT CARE CENTER. M. Khan ${ }^{1}$, A. Evans ${ }^{1}$, S. Vargas ${ }^{1}$, N. Brahmbhatt ${ }^{1}$, M. Rafiq ${ }^{1}$, K. Perumal $^{1}$, F. Daraiseh ${ }^{1}$, C. Chataut ${ }^{1}$, W. Ahmed ${ }^{1}$, R. Kumapley ${ }^{1}$, L. Fogelfeld ${ }^{1}$; ${ }^{1}$ Cook County Hospital, Chicago, IL (Tracking ID \#76860)

BACKGROUND: Urgent care centers at safety-net hospitals are often the sole source of care for minority patients with chronic illnesses. Our goals were to determine how often patients with diabetes seek care at an urgent care center of a large public hospital and assess the need for more comprehensive services for diabetic patients within this setting.

METHODS: We conducted a survey of consecutive diabetic patients presenting to the Ambulatory Screening Clinic of Cook County Hospital during a representative 14 day period. Physicians interviewed subjects using a standardized data collection instrument and recorded information on demographic and clinical characteristics, medication use, diabetes knowledge, self-management practices, and co-morbidities. Glycosylated hemoglobin (A1c) and serum creatinine were also measured for all subjects.

RESULTS: Of the 3044 patients cared for in the urgent care clinic during the 14 consecutive days, 410 had diabetes (prevalence: $13 \%$ ). Thus, 10700 patients with diabetes are expected to present to the clinic yearly. The clinical characteristics of the sample were: $90 \%$ of these patients were Type 2 diabetics. $80 \%$ were overweight $(\mathrm{BMI}>25$ ). Low proportions,about one third were taking metformin in a population with high prevalence of obesity. $80 \%$ had rudimentary diabetic knowledge. Only 4\% were aware that an A1c of 10 was undesirable. Only $33 \%$ reported taking their medications regularly and check their blood sugar consistently. Significantly $18 \%$ could not read the label of a drug prescription bottle, one fourth needed interpreter services, and two thirds had no primary care physician. The mean A1c was $9.2(\mathrm{SD} \pm 2.4)$.

CONCLUSION: Diabetes Mellitus is a prevalent problem in the urgent care center of a large, urban, public hospital. The patients' demographic and clinical characteristics suggest that comprehensive services are necessary to meet their needs, including more interpreters and diabetes educators. These services need to address the problem of obesity, low utilization of metformin, and improve self-management skills. Urgent care centers at safety-net hospitals serving predominately poor, minority populations - must adapt to a clear health care need: comprehensive care for chronic diseases such as diabetes.

UNIQUE NEEDS OF HIV-INFECTED HOMELESS PERSONS. T.W. Kim ${ }^{1}$, N.J. Horton ${ }^{1}$, J.H. Samet ${ }^{1} ;{ }^{1}$ Boston University, Boston, MA (Tracking ID \#75696)

BACKGROUND: Despite HIV-infected persons' increased mortality, characterization of HIV-infected homeless persons has been limited apart from adherence issues. We analyzed a cohort of HIV-infected persons with a history of alcohol problems to compare clinical and utilization differences between the homeless and the housed.

METHODS: A standardized questionnaire was administered to $349 \mathrm{HIV}$-infected participants entering the HIV-Alcohol Longitudinal Cohort (HIV-ALC) study. In bivariate analyses, homelessness (at least 1 night in a shelter or on the street in the past 6 months) was examined with regard to the following outcome variables: CD4 count, current antiretroviral (ART) therapy, HIV-related symptoms (in past 30 days, range $0-12$ ), delay of care (months between the 1 st positive HIV test and 1st HIV primary care visit), utilization of emergency department (ED) and hospitalization (any vs. none for past 6 months), and alcohol drinks per day (in past 30 days). RESULTS: Despite similar CD4 counts between the housed and the homeless, significant differences were found (see Table below). Homelessness was associated with less ART use, more HIV-related symptoms, delayed HIV primary care, more utilization and higher alcohol consumption.

CONCLUSION: Among a cohort of HIV-infected persons with a history of alcohol problems, homelessness was associated with more symptoms, less optimal self-care, and higher utilization despite similar CD4 counts to housed persons. Our findings support the case for specialized services for homeless HIV persons.

$\begin{array}{lccl}\text { CD4 count }(\text { cell/ } \mu \mathrm{l}) & 399 & 407 & 0.80 \\ \text { current ART } & 64 \% & 46 \% & 0.003 \\ \text { HIV symptoms } & 7.3 & 8.7 & 0.0004 \\ \text { delay (months) } & 11.5 & 18.7 & 0.04 \\ \text { any ED } & 25 \% & 39 \% & 0.0009 \\ \text { any hospitalization } & 27 \% & 39 \% & 0.03 \\ \text { mean drink/day } & 1.6 & 5.4 & 0.03\end{array}$

RACIAL DISPARITY IN SHORT- AND LONG-TERM OUTCOMES AFTER PERCUTANEOUS CORONARY INTERVENTION. S.H. Konety', M.V. Sarrazin ${ }^{2}$, G.E. Rosenthal ${ }^{1} ;{ }^{1}$ University of lowa Hospitals and Clinics, lowa City, IA; ${ }^{2}$ VAMC lowa City, lowa City, IA (Tracking ID \#76599)

BACKGROUND: Although prior studies have documented racial disparity in the use of percutaneous coronary intervention (PCI) following acute myocardial infarction (MI), fewer studies have examined racial differences in short- and long-term mortality after PCI. METHODS: We reviewed Medicare claims data on 553,727 patients aged 65 and older who underwent PCI from 1994 through 1997. We compared both short-term (in-hospital or within 30 days of PCI) and long-term mortality (90 days and 365 days of PCI) for black and white patients. Analyses adjusted for age, gender, comorbidity, admission source and urgency, prior coronary bypass surgery or PCI, and concurrent MI. We then preformed separate stratified analysis by gender to determine if the relationship between race and mortality after PCI was similar in males and females.

RESULTS: Blacks were younger (mean ages 72 vs. 74 years; $\mathrm{p}<.001$ ) and more likely $(\mathrm{p}<.001)$ to be female $(55 \%$ vs. $45 \%)$ and to have diabetes $(17 \%$ vs. $7 \%)$, peripheral vascular disease $(7 \%$ vs. $6 \%)$, congestive heart failure $(17 \%$ vs. $13 \%)$, and a recent MI ( $32 \%$ vs. $30 \%)$. Blacks were less likely (p <.001) to have COPD ( $8 \%$ vs. $10 \%)$, or a history of previous CABG $(6 \%$ vs. $10 \%)$ or PCI (5\% vs. $7 \%$ ). Unadjusted 30 -day (3.5\% vs. $3.6 \%$ ) and 90 -day ( $5.0 \%$ vs. $4.8 \%)$ mortality was similar in blacks and whites, although 365 -day mortality was higher in blacks $(9.6 \%$ vs. $8.5 \% ; \mathrm{p}<.001)$. However, after adjusting for patient-level factors, the odds of death for blacks, relative to whites, was lower $(\mathrm{p}<.001)$ at both 30 days $(\mathrm{OR}=0.88 ; 95 \% \mathrm{CI}, 0.82-0.95)$ and 90 days $(\mathrm{OR}=0.92 ; 95 \% \mathrm{CI}, 0.87-0.98)$ and was similar at 365 days $(\mathrm{OR}=1.03 ; 95 \% \mathrm{CI}$, 
$0.98-1.08)$. These results were consistent in separate analyses of males (30-day OR $=0.87$; $95 \% \mathrm{CI}, 0.78-0.98$ ) and females (30-day OR $=0.89 ; 95 \% \mathrm{CI}, 0.81-0.98$ )

CONCLUSION: Among Medicare patients undergoing PCI, we found no evidence for racial disparity in either short-term or long-term mortality. While our analyses may be confounded by unmeasured severity or by selection bias in referral for PCI, the results suggest that previously described disparity in outcomes after coronary bypass surgery may not be generalizable to other invasive cardiac procedures.

EVALUATION OF PATIENT SATISFACTION IN A MEDICATION ASSISTANCE PROGRAM (MAP). A. Kwon ${ }^{1}$, S. Peters ${ }^{2}$, J. Lopez ${ }^{2}$, J. Diaz ${ }^{3}$, M. Fagan ${ }^{2}$; ${ }^{1}$ Brown Medical School, Providence, RI; ${ }^{2}$ Rhode Island Hospital, Providence, RI; ${ }^{3}$ Memorial Hospital of Rhode Island, Pawtucket, RI (Tracking ID \#76872)

BACKGROUND: The high cost of prescription medications has led to the widespread institution of MAPs, pharmaceutical sponsored programs which provide free medications to indigent patients. The MAP at our internal medicine residents' clinic is administered by a full time nurse. We sought to describe the demographic characteristics and to identify factors correlating with MAP enrollee satisfaction.

METHODS: From August to December 2002, MAP-enrolled patients were recruited. The interviewer-administered questionnaire assessed socio-demographics, medical history, access to medications, gaps in medication usage, frequency of medication changes, and attitude to the services provided by the MAP. Patient attitudes were measured using Likert-type scales where $1=$ "Strongly agree" and $5=$ "Strongly disagree". Data were analyzed using T-test and Chisquare statistics. We then constructed a multi-variable model to identify potential predictors to the outcome variable of overall satisfaction.

RESULTS: 77 patients were interviewed. The average age was $57.4 \pm 11.2$ years, and $51 \%$ were male. $47 \%$ were White, $27 \%$ were Hispanic, and $18 \%$ were Black. $50 \%$ had health insurance, although $82 \%$ of those with insurance were Medicare enrollees. $73 \%$ had an annual income less than $\$ 15,000$, and $75 \%$ had completed high school or its equivalent. There were no significant racial differences in income or education. Hispanic enrollees were significantly less likely to have health insurance than both Blacks and Whites $[\mathrm{OR}=.23,95 \% \mathrm{CI}(.07, .72)]$. Black and Hispanic enrollees took less medications on average than Whites (3.9 and 3.7 vs. $5.2, \mathrm{P}=.01$ ), but Hispanic enrollees had a higher proportion of their medications covered by the MAP compared to Whites $(.97$ vs. $.84, \mathrm{p}=.01)$. All participants were generally very satisfied with the services provided by the MAP (mean $=1.18 \pm .45$ ), with no differences across race or income. $80.5 \%$ of participants felt their general health improved since MAP enrollment. $74 / 77$ (96.1\%) expressed no major delays in receiving their medications through the program. Insignificant gaps in medication usage, self-perceived better health since enrollment, and higher income significantly predicted satisfaction with the MAP $(r=0.61$, $\mathrm{F}<.0001)$.

CONCLUSION: MAP-enrolled patients represent an ethnically diverse population, half of which have some form of health insurance. Most patients expressed a high degree of satisfaction with the MAP and felt their health to be positively affected by their participation. Patient satisfaction as correlated with little interruption in medication supplies may be attributed to the administrative support provided through our institution. Further studies are necessary to evaluate the specifics of institution-facilitated support in promoting satisfaction with MAPs.

PATIENT LITERACY, ADHERENCE AND ANTICOAGULATION THERAPY OUTCOMES A PRELIMINARY REPORT. L. Lasater ${ }^{1}$, A. Davidson ${ }^{1}$, P. Mehler'; ${ }^{1}$ Denver Health, Denver, CO (Tracking ID \#76507)

BACKGROUND: Literacy's likely impact on adherence is poorly understood. Measurement of literacy may help guide decisions regarding initiation of treatment as well as anticipate those at increased risk for adherence problems. Anticoagulation therapy provides a good model for assessing adherence and outcomes, given known complications, and ease of monitoring (INR).

METHODS: Design: Cohort study of low-income anticoagulation clinic (AC) patients at Denver Health (DH). Electronic data were collected from AC computer records (visits, INR results, weekly dosage) and DH pharmacy files (warfarin dispensed [WD]). Pharmacy profiles ( $\mathrm{mg}$ of $\mathrm{WD}$ ) were divided by prescribed $\mathrm{AC}$ dosage to estimate adherence. Language concordant (English/Spanish) patient interview included: demographic factors, Test of Functional Health Literacy in Adults (TOFHLA, max. score $=36$ ) for literacy and an AC Knowledge Questionnaire (KQ) generated from verbal, written, and audiovisual educational materials (maximum score $=11$ ). Barriers to care (i.e., no phone, car, answering machine, childcare) were summed for an overall barrier index. Numeric variables presented with $\pm S D$; comparisons by Pearson correlation or Student T-tests.

RESULTS: 196 AC patients, with more than one WD and interview completed were followed for 323 person-years (mean: 1.65 years, \pm 1.0 ); mean INR performed per patient: $18, \pm 11$; mean prescriptions WD: $8.4 \pm 5$; mean adherence: $0.75 \pm .21$; mean TOFHLA: $23.9 \pm 11.7$; mean KQ $8.4 \pm 2.3$; mean barriers: $1.34 \pm 1.0$. Limited by current study enrollment, literacy level and adherence trended to statistical correlation $(\mathrm{p}=0.11)$. Literacy level was positively correlated with the percentage of INRs within therapeutic range $(p=0.03)$ and higher KQ scores ( $\mathrm{p}<0.0001)$. Among Hispanics $(\mathrm{N}=67)$, Spanish-speakers were more likely to have marginal or inadequate literacy compared to English-speaking subjects (mean TOFHLA score 13.6 vs. 26.2 respectively, $\mathrm{p}=<0.0001)$ and higher barrier scores $(\mathrm{p}<0.02)$. An inverse correlation was observed between barriers and TOFHLA and KQ scores $(\mathrm{p}<0.005)$ and an inverse trend $(\mathrm{p}=0.09)$ between barriers and adherence.

CONCLUSION: Literacy was associated with better rates of anticoagulation control and greater knowledge of AC issues. Among Spanish speakers, both adherence measures and literacy levels were lower. Educational materials should address the needs of high-risk patients with poor health literacy.
HEALTH CARE FOR HOMELESS WOMEN; UNMET NEEDS AND BARRIERS TO CARE. J.H. Lewis ${ }^{1}$, L. Gelberg ${ }^{1}$, R. Andersen ${ }^{1} ;{ }^{1}$ University of California, Los Angeles, Santa Monica, CA (Tracking ID \#76824)

BACKGROUND: Homelessness is a significant and growing problem in the United States. Women and families are the fastest growing segments of the homeless population. Living without a home increases one's risk of having health problems and of experiencing barriers to care. This study determines how much perceived unmet need for medical care there is among homeless women, what homeless women perceive to be barriers to health care and how barriers and other factors are associated with unmet needs.

METHODS: Cross-sectional study of homeless women, utilizing structured interviews from a community based probability sample of 974 homeless women aged 15 to 44 years. We measured perceived unmet need for medical care in the past 60 days. Determined the relationship between unmet need and demographic variables, place of stay, source of health care, insurance and perceived barriers to care.

RESULTS: Of the 974 women, $37 \%$ reported unmet need for medical care. Controlling for other factors the odds of unmet need were lower among those with a regular source of care (OR .35, 95\% confidence interval [CI] .21 to .59), while having health insurance was not significantly associated. The odds of unmet need were higher among those who experienced the barriers: not knowing where to go (OR 1.97, CI 1.21 to 3.20 ), long office waiting times (OR 1.75, CI 1.16 to 2.63 ) and being too sick to seek care (OR 1.89 CI 1.12 to 3.18).

CONCLUSION: There is significant unmet need for medical care among homeless women. Having a regular source of care was more important than health insurance in lowering the odds of unmet need. We have identified important barriers to care for this population. Homeless women must be educated regarding sources of care and clinics serving the homeless must decrease waiting times. Increased outreach to homeless women to help them stay well or to help them address problems early could decrease unmet need in this vulnerable population.

CITIZENSHIP STATUS IS AN IMPORTANT DETERMINANT OF HEALTH CARE DISPARITY. A.K. Li ${ }^{1}$, K.E. Covinsky'; ${ }^{1}$ San Francisco VAMC, University of California, San Francisco, San Francisco, CA (Tracking ID \#74299)

BACKGROUND: Immigrants account for over $10 \%$ of the U.S. population and around a third of the immigrants in United States reside in California. We examined whether differences of care exist between U.S. born, naturalized U.S. immigrants (ie. immigrants who obtained their U.S. citizenship) and non-U.S. immigrants in California.

METHODS: We analyzed data from the 2001 California Health Interview Survey, a population based telephone survey designed to assess the health status of Californians over the age of 18. Outcome measures included the use of prescription anti-hypertensive and diabetic medications for persons diagnosed with hypertension and diabetes, respectively, and contact with mental health specialist for those who desired mental health care. Logistic regression were used to discern the independent effects of a person's citizenship status and each of these outcomes. All analyses were adjusted for the survey sample weights to derive estimates of California's general population.

RESULTS: $77 \%$ of Californians were U.S. born, $11 \%$ were naturalized U.S. immigrants and $12 \%$ were non-U.S. immigrants. The mean age for U.S born, naturalized U.S. immigrants and non-U.S. immigrants were 50, 49 and 38 years of age, respectively. Non-U.S. immigrants were more likely to be uninsured compared to naturalized U.S. immigrants and U.S. born citizens $(40 \%$ vs $14 \%$ vs. $10 \%$; P < 0.001$)$. In comparison to U.S. born and naturalized U.S. immigrants diagnosed with hypertension and diabetes mellitus, significant fewer non-U.S. immigrants received medications for hypertension $(67 \%$ vs. $67 \%$ vs. $41 \%$; $\mathrm{P}<0.001)$ and diabetes mellitus ( $78 \%$ vs. $80 \%$ vs. $60 \% ; \mathrm{P}<0.001)$. Similarly, among those who desired mental health services, significantly fewer non-U.S. immigrants received mental health care than naturalized U.S. immigrants and U.S. born citizens ( $49 \%$ vs. $33 \%$ vs. $14 \%$; $\mathrm{P}<0.001)$. After adjusting for age, gender income, education, race, insurance status, years in the U.S ( $<10$ and $\geq 10 \mathrm{yrs})$ and ability to speak English, we determined that naturalized U.S. citizens were just as likely as U.S. born citizens to receive medications for hypertension (OR $1.0 ; .95 \%$ C.I. .9-1.2), and diabetes (OR .9; $95 \%$ C.I. .6-1.2). Naturalized immigrants were only slightly more likely not to see a mental health specialist (OR 1.6; 95\% C.I. 1.3-2.0) than U.S. born citizens. Moreover, while non-U.S. immigrants were nearly as likely as U.S. born citizens to receive anti-hypertensive medications (OR 1.2; 95\% C.I. 1.0-1.6), non-U.S. immigrants were more likely not to receive diabetic medications (OR 1.8; 95\% C.I. 1.2-2.7) and more likely to not utilize mental health services (OR 2.6; $95 \%$ C.I. 1.9-3.4) than U.S. born citizens.

CONCLUSION: Non-U.S. immigrants are more likely not to receive medications for hypertension, diabetes and access mental health services than U.S. and naturalized citizens even after adjusting for potential confounders. However, observed differences in health care disparity appear to minimize once a foreign born immigrant becomes an U.S. citizen.

PHYSICAL AND SEXUAL ABUSE IN A COHORT OF HIV-INFECTED PERSONS WITH ALCOHOL PROBLEMS: CURRENT AND LIFETIME PREVALENCE AND ASSOCIATION WITH HEALTH CARE UTILIZATION. J.M. Liebschutz ${ }^{1}$, J.L. Geier ${ }^{1}$, N.J. Horton ${ }^{1}$, C.H. Chuang ${ }^{1}$, J.H. Samet ${ }^{1} ;{ }^{1}$ Boston University, Boston, MA (Tracking ID \#74638)

BACKGROUND: While cross-sectional studies have shown that physical and sexual abuse histories are associated with increased health care utilization, they rarely controlled for comorbidities and primarily studied women. We examined the relationship between current and past physical and sexual abuse and health care utilization among a longitudinal cohort of HIVinfected persons.

METHODS: The HIV-Alcohol Longitudinal Cohort (HIV-ALC) study recruited 349 HIVinfected subjects with a history of alcohol problems and followed them every 6 months for up to 36 months. At study entry, assessment included demographics, lifetime physical and sexual abuse histories (none, physical only [Physical Abuse], sexual with or without physical [Sexual 
Abuse]) and age at first abuse (childhood abuse defined as $<13$ years old). At study entry and each follow-up, subjects were assessed for occurrence in the prior 6 months of the following: physical or sexual abuse [Current Abuse], substance abuse, depressive symptoms, emergency department (ED) visits and hospitalizations. We used the Kaplan-Meier survival estimator to calculate the proportion of subjects reporting current abuse over time. Generalized estimating equation regression models evaluated the relationship between abuse (lifetime, childhood, and current) and utilization measures over time while controlling for age, gender, education, and ethnicity, as well as recent substance abuse, depressive symptoms, and housing status.

RESULTS: HIV-ALC subject characteristics were: $79 \%$ male; mean age $41 \mathrm{yrs} ; 44 \%$ black, $33 \%$ white, and $23 \%$ other. Most $(80 \%)$ reported lifetime physical and/or sexual abuse: $40 \%$ Physical Abuse, and 40\% Sexual Abuse. Childhood abuse was reported by $46 \%$. By 18 and 30 months, $15 \%$ and $36 \%$ of the sample, respectively, had reported current abuse. In bivariate analyses, women experienced more sexual abuse $(73 \%$ vs. $32 \%)$ and more current abuse $(16 \%$ vs. $7 \%$ at entry) than men. In multivariate analyses, current abuse was significantly associated with hospitalization $(\mathrm{OR}=1.23, \mathrm{CI}=1.01-1.51)$. Current abuse was borderline significantly associated with $\mathrm{ED}$ visits $(\mathrm{OR}=1.27, \mathrm{CI}=0.96-1.68)$. Lifetime and childhood abuse were not significantly associated with ED visits or hospitalizations $(\mathrm{p}>0.10)$.

CONCLUSION: A history of physical or sexual abuse is widespread among HIV-infected persons with a history of alcohol problems. Current abuse was common and independently associated with increased hospitalization. Care providers should consider violence exposure in their HIV-infected patients with a history of alcohol problems. Effective violence prevention interventions may benefit these high risk patients.

UNDERSTANDING THE EFFECTS OF RACIAL RESIDENTIAL SEGREGATION ON HEALTH STATUS. D.G. Litaker ${ }^{1}$, J.J. Sudano ${ }^{2}$, N. Colabianchi ${ }^{1} ;{ }^{1}$ Case Western Reserve University, Cleveland, $\mathrm{OH} ;{ }^{2}$ Center for Healthcare Research and Policy, Cleveland, $\mathrm{OH}$ (Tracking ID \#76804)

BACKGROUND: Health status may be influenced by characteristics of the individual and the environment in which they live. Although racial residential segregation (RRS), an important contextual attribute, has been associated with mortality, its relationship with general health status has not been fully examined. The specific aims of this study, therefore, were to: 1) assess the magnitude of association between RRS and health status, 2) evaluate the extent to which this relationship was mediated through characteristics of the individual and their environment, and 3) determine whether this association varied by age, gender, educational attainment, or insurance status.

METHODS: Data from the Ohio Family Health Study, a cross sectional survey assessing insurance coverage, health care utilization, and health status, were linked with contextual data from the Area Resource File. Guided by Andersen's conceptual framework of access, this analysis used nested hierarchical models to quantify the association between RRS and health status alone and after adjusting for individuals' enabling (e.g., insurance status, social support, income), predisposing (e.g., age, sex, race, education), and need-related characteristics (e.g., number of chronic medical conditions, rating of general health status) and contextual attributes (e.g., unemployment rate, community economic status, population density, proportion of racial/ethnic minority residents, primary care physician supply, and managed care activity). Selected cross-level interactions were assessed.

RESULTS: Of 15,613 adults in 623 areas, 2390 (15.3\%) reported their general health as "fair" or "poor". In a model without other factors, RRS was associated with lower health status $(\mathrm{OR}=1.15,95 \% \mathrm{CI}[1.08,1.21] ; \mathrm{p}<.001)$. Adding other contextual attributes attenuated the significance of the association between segregation and health status $(\mathrm{AOR}=1.00[0.95,1.06])$. In a final model including individuals' enabling, predisposing, and need characteristics, segregation remained statistically unassociated with health status $(\mathrm{AOR}=1.01[0.98,1.05]$; c statistic $=0.862)$. We observed no significant cross-level interactions between RRS and individual characteristics.

CONCLUSION: Although greater racial segregation is associated with lower health status, contextual characteristics and attributes of the individual may mediate much of this effect. These findings hint at an important dynamic, the elements of which should be studied more closely to better inform policy solutions for reducing apparent effects from this fundamental cause of health disparities.

RACIAL SENSITIVITY AMONG FIRST AND SECOND YEAR MEDICAL STUDENTS. J.A. Long ${ }^{1}$, A. Gomez ${ }^{2}$, M. Cox $^{3}$, D.A. Asch ${ }^{1}$, J.A. Shea ${ }^{3} ;{ }^{1}$ VA Center for Health Equity Research and Promotion, Philadelphia, PA; ${ }^{2}$ University of Pennsylvania, Philadelphia, PA; ${ }^{3}$ University of Pennsylvania School of Medicine, Philadelphia, PA (Tracking ID \#75753)

BACKGROUND: There is growing evidence that physicians contribute to racial and ethnic disparities in health care. Few studies, however, have evaluated medical student bias or whether these biases differ by years of medical education completed.

METHODS: We surveyed 1st and 2nd year medical students' selection preferences for candidates for a NIH funded research scholarship and a research assistant position with a private biomedical company. Students received a packet with four identical curriculum vitae (CVs) with photographs attached. Three of the CVs were identical for all students. One CV described a candidate (Eric Johnson) who was portrayed as African American (AA) in half the students' packets, and as White for the other half. Question and CV order were varied from packet to packet. Students' ratings of the candidates on a $0-10$ scale were converted to rankings with one being the highest and four the lowest. T-tests were performed to identify differences in the ranking between the AA and White Eric Johnson.

RESULTS: Over $75 \%$ of both classes completed the survey (122 first and 110 second year students). Forty eight percent of the students were female, and $22 \%$ were under-represented minorities. There were no significant differences in the ranking between the AA and White candidate for the private sector position. However, the AA candidate received a better mean score than the White candidate for the scholarship (2.10 and 2.37 respectively; effect size 0.47 , $\mathrm{p}<0.001$ ). By class, the difference in mean scores was statistically different only for 2 nd year students (mean score for the AA compared to the white candidate 2.14 versus 2.47; effect size $0.51, \mathrm{p}<0.001)$. However, both 1 st and 2 nd year under-represented minority students were more likely to prefer the AA candidate. Among under-represented minority students the mean score for the AA candidate was 2.02 compared to 2.60 for the White candidate (effect size 1.01, $\mathrm{p}<0.001)$.

CONCLUSION: We found no evidence of reduced preference for the hypothetical AA student in this sample of medical students. In contrast, we found a significant preference for the AA student among second year medical students and among students themselves from underrepresented minorities. This effect was observed only for the scholarship and not the research position, suggesting these findings are sensitive to context. One might expect the preference by under-represented minorities for the AA candidate. Causes of the difference between first and second year students are less obvious. Among the possible explanations are increased clinical and academic maturity and exposure to a cultural sensitivity curriculum during the first year of medical school.

OCCUPATION CLASSIFICATION: NO EASY SHORT CUTS. J.A. Long ${ }^{1}$, M.I. Bamba ${ }^{1}$, N.R. Kressin ${ }^{2} ;{ }^{1}$ VA Center for Health Equity Research and Promotion, Philadelphia, PA; ${ }^{2}$ VA Center for Health Quality, Outcomes and Economic Research, Bedford, MA (Tracking ID \#75851)

BACKGROUND: Socioeconomc Status (SES) is frequently measured in multiple ways including education completed, income, and occupation. In epidemiologic surveys, however, occupation is frequently not asked about since classifying occupations into coherent groups that are used for analyses is labor intensive. Relying on study participants to classify their own occupations could be a significant labor saving process. The objective of this study, therfore, was to determine how accurately individuals can classify themselves into standard occupational groups.

METHODS: The data used in these analyses are from the pilot baseline interview of the VA Physician Intervention to Improve Hypertension study. Veterans were first asked to answer the question "What kind of work have you done most of your life?" After this question, they were asked to categorize the work into a standard Major Occupational Group (MOG) from the Occupational Classification System created by the Bureau of Labor Statistics. The primary investigator (PI) then independtly categorized each reported occupation using the 1990 Census Occupation Index Listings. Kappa scores were calculated to determine how well the veterans and the PI agreed.

RESULTS: Ninety two of 96 people responded to both questions. For 78 people (85\%), we were able to assign them to a MOG given the occupation title provided. Eight people (9\%) provided an occupation title that was too vague to be able to assign them to a MOG. Six people (6\%) gave "military" or a military occupation as their occupation title (military occupations are not included in the Occupational Classification System). For the 78 veterans that provided an occupation title contained within the Occupational Classification System, only 42 (54\%) correctly identified the MOG in which their occupation was grouped based on the title proved. The weighted kappa coefficient for agreement between the veterans and the PI was 0.51 (95\% CI $0.37-0.65)$.

CONCLUSION: Veterans were only able to accurately classify their occupation into a MOG about $50 \%$ of the time. In addition, job titles were frequently inadequate for the PI to classify an individual's occupation, especially for veterans in military occupations. For researchers interested in collecting data on occupations, relying on study subjects to classify their occupations into an occupational group is not adequately accurate. In addition, just asking for an occupation title frequently does not provide enough information for researchers to accurately code occupations especially for those in military professions.

MISSING RACE DATA IN VA BASED DISPARITIES RESEARCH: A SYSTEMATIC REVIEW. J.A. Long ${ }^{1}$, B.I. Melissa', J.A. Shea ${ }^{2} ;{ }^{1}$ VA Center for Health Equity Research and Promotion, Philadelphia, PA; ${ }^{2}$ University of Pennsylvania School of Medicine, Philadelphia, PA (Tracking ID \#76904)

BACKGROUND: Many studies evaluating racial disparities come from the VHA and are based on secondary data analyses. Often race data are missing for at least a portion of the patients. Knowing how investigators treat missing data is critical in evaluating potential biases. The objectives of this systematic review were to quantify: (1) the data sources for VHA disparity studies; (2) how missing data were handled; and (3) the extent of missing data.

METHODS: MEDLINE, EconLit, and Sociological Abstracts were searched using these keywords: (race, racial stock, ethnicity, ethnic groups, blacks, Hispanic Americans) and (United States Department of Veterans Affairs, veterans, veterans hospitals, and VA). Abstract exclusion criteria included: written before 1992; letters or review papers; did not pertain to veterans; race not mentioned; and race self-reported. Two trained reviewers independently abstracted each article. Article exclusion criteria included: duplicate study populations; not a secondary data analysis; race was not a focus of or important predictor in the research. Information abstracted from each article included, among others, the role of race in the study, the source of the race data, how missing race data were handled and the quantity of missing race data.

RESULTS: 68 of 118 articles met inclusion criteria. Race was the primary focus in 42 articles and an important predictor in 27 articles. The Patient Treatment File (PTF) was the most common source of race data (29). For 32 articles knowledge of race was required for inclusion in the analytic population. Articles were grouped into the following mutually exclusive categories: no missing race data (11); missing race data explicitly quantified (8); missing race data explicitly grouped with other data but not quantified (9); race data known for enumeration of the potential population (2); no mention of missing race data but known to exist in the data source e.g., PTF (5); unable to determine if there was missing race data (33). When missing race data was quantified it ranged from $0 \%$ to $48 \%$, median $=0 \%$, mean $=8 \%$. 
CONCLUSION: Missing race data is frequently present in VHA secondary data sources. However, it is rarely explicitly discussed or quantified, even when it is the primary focus of the research question. Without clear descriptions of how much missing race data is present in studies using VHA secondary data sources, readers are unable to evaluate a very important potential source of bias.

R-E-S-P-E-C-T: PATIENT REPORTS OF DISRESPECT IN THE HEALTHCARE SETTING AND ITS IMPACT ON CARE. $\underline{\text { N. Lurie }}{ }^{1}$, J. Blanchard ${ }^{2} ;{ }^{1}$ RAND, Arlington, VA; ${ }^{2}$ George Washington University, Washington, DC (Tracking ID \#76201)

BACKGROUND: The patient-doctor (pt-dr) relationship has been pinpointed as a domain in which racial/ethnic disparities may arise, and is a possible area for intervention. We hypothesized that patients (pts) who experience discomfort or disrespect in the relationship may be less likely to initiate or follow through with needed care.

METHODS: We analyzed data from the Commonwealth Fund Survey of Disparities in Quality of Health Care. It included 6722 adults age 18 and over (53\% response rate) weighted to form a nationally representative sample and was conducted in 6 languages. Pts reported about their experiences with care. We examined whether the pt felt s/he had been treated with respect or looked down upon, whether s/he felt treated unfairly because or race, ability to pay, gender or language, and whether $\mathrm{s} / \mathrm{he}$ felt they would have received better care if $\mathrm{s} / \mathrm{he}$ had they been of a different race or gender, and whether their doctor understood their values. Outcomes included: putting off care, following the doctor's advice, and receipt of a physical exam in the prior year, age-appropriate cancer screening, and 'optimal care' for pts with diabetes (HbA1, eye, foot, cholesterol and BP check), hypertension and heart disease (BP check and cholesterol). All analyses used chi-squared tests and multivariate logistic regression, and were performed in STATA.

RESULTS: $15 \%$ of Blacks, $21 \%$ if Hispanics and $22 \%$ of Asians reported being treated with disrespect or being looked down upon in the past, versus only $10 \%$ of whites $(p<0.01)$. A significant proportion of minorities also reported having been treated unfairly because of race and that they would have received better treatment if they had been a different race (all $\mathrm{p}<.05$ compared with whites). Independent of race or insurance status, pts who felt that had been treated with disrespect, treated unfairly due to race, or that they would have received better care if they were a different race were less likely to receive optimal chronic disease care and more likely to put off care or not follow the doctor's advice (all $\mathrm{p}<0.05$ ). For example, only $58 \%$ of those who felt that they had been treated with disrespect reported optimal chronic disease care and $30 \%$ put off care, vs. $75 \%$ and $18 \%$ of those who did not, $p<0.01$. Of those who felt treated unfairly because of their race, $46 \%$ did not follow the doctors advice, and $40 \%$ put off care, vs. $23 \%$ and $20 \%$ of those who did not, $(\mathrm{p}<.001)$. This trend was largely similar for those who felt that they would have received better care if they belonged to a different race. CONCLUSION: Independent of race and income, pts reports of how they are treated in the pt-dr relationship are strongly related to getting needed care. Pts who report discomfort with the relationship are more likely to put off care and less likely to follow advice. Controlling for other factors, minority pts report more problematic pt-dr relationships. Strategies to address these issues need to be developed.

RACE AND AGE DISPARITIES OF PSA USE (SHOULDN'T WE PRACTICE WHAT WE PREACH?). R. Mansfield ${ }^{1}$, R. Velez ${ }^{1}$, G.J. Chen ${ }^{1}$, W.P. Morān ${ }^{1} ;{ }^{1}$ Wake Forest University, Winston-Salem, NC (Tracking ID \#76714)

BACKGROUND: The incidence of prostate cancer in African-Americans is 1.6 times that of Caucasians, and curable cases of prostate cancer are most likely to occur in men $<70$ yrs old Currently there is no published national data that suggest PSA screening is targeting these groups.

METHODS: The National Health Interview Survey (NHIS) is an annually performed selfreport multistage-probability designed survey intended to represent the non-institutionalized U.S. population. The 2000 NHIS cancer module includes specific questions on PSA use. The SUDAAN software was used to produce weighted estimates of PSA use for screening purposes within the last 12 months stratified by age and race adjusting for cluster and sampling effects. RESULTS: Results are provided in the table.

CONCLUSION: The majority of men for whom early detection of prostate cancer is most likely to be beneficial (age $<70$ ) are less likely to have been screened than older men, for whom screening is more controversial. Black men, despite the higher risk of prostate cancer, are not screened with higher frequency past age 50 than White men. Thus, the present use of the PSA does not reflect currently promoted screening guidelines regarding age and race.

\begin{tabular}{lccccc} 
& \multicolumn{5}{c}{ Results } \\
& BLACK & WHITE & OR & $\mathbf{9 5 \%}$ Cl & p-value \\
age $45-49(\mathrm{n})$ & 108,6221 & $7,962,365$ & & & \\
-psa in last $12 \operatorname{mos}(\%)$ & $15.0 \%$ & $9.8 \%$ & 1.63 & $1.00-2.65$ & 0.0782 \\
age $50-69(\mathrm{n})$ & $2,043,928$ & $20,226,898$ & & & \\
-psa in last $12 \operatorname{mos}(\%)$ & $31.1 \%$ & $29.6 \%$ & 1.08 & $0.83-1.40$ & 0.5933 \\
age $>70$ & 715,832 & $8,761,137$ & & & \\
-psa in last $12 \operatorname{mos}(\%)$ & $25.7 \%$ & $34.7 \%$ & 0.65 & $0.43-0.99$ & 0.0334
\end{tabular}

THE RELATIONSHIP BETWEEN PATIENT LITERACY AND KNOWLEDGE, ATTITUDES, AND BEHAVIOR REGARDING OBESITY. E. Marin ${ }^{1}$, J. Huang ${ }^{1}$, H. Yu ${ }^{2}$, T.C. Davis ${ }^{1}$, C. Arnold ${ }^{1}$, D. Carden ${ }^{1}, \mathrm{~S}$. Brock ${ }^{1}$; ${ }^{1}$ Louisiana State University Medical Center at Shreveport, Shreveport, LA; ${ }^{2}$ Yale University, New Haven, CT (Tracking ID \#76340)

BACKGROUND: Reducing the prevalence of obesity and improving health literacy are both national health objectives in Healthy People 2010. Low literacy may undermine a patient's ability to understand the adverse health effects of obesity and the need for weight loss. The purpose of this study was to determine the relationship between the literacy level of overweight/obese patients and their weight-related knowledge, attitude, and behavior.

METHODS: Structured patient interviews and a literacy screening instrument, the Rapid Estimate of Adult Literacy (REALM), were administered in two primary care clinics at a university-based public hospital. A previous study found that $81 \%$ of patients at these clinics are overweight or obese. A convenience sample of 210 overweight or obese outpatients (with a body mass index (BMI) of $\geq 25$ ), $\geq 18$ years old, were enrolled.

RESULTS: Mean respondent age was 52 (range $18-92$ ). $74 \%$ were women; $76 \%$ percent were black and $24 \%$ were white. Thirty percent were Medicaid or Medicare recipients and $4 \%$ were commercially insured. The mean BMI for the study population was 38.9 (range 26-65). $8 \%$ of patients were overweight (BMI 25-29.9), and 92\% obese (BMI 30+). Patient literacy was low. $67 \%$ read below a 9 th grade level $(33 \% \leq 6$ th grade; $35 \% 7$ th- 8 th grade). Literacy levels were significantly lower for older patients $(\mathrm{p}<.02)$, blacks $(\mathrm{p}<.0001)$, and Medicaid/Medicare recipients $(\mathrm{p}=.0003)$. Only $47 \%$ of patients could read all seven weight-related words (i.e., fat, meals, exercise, calories, nutrition, diabetes, obesity) on the REALM. $50 \%$ of patients across all literacy levels reported currently attempting weight loss. There was a significant relationship between literacy level and weight-related knowledge, attitudes, and behavior, summarized below.

$\begin{array}{lcclc} & <6 \text { th } \text { grade } 7^{\text {th }}-8^{\text {h }} \text { grade } & \text { High School } & p \\ \text { Understand weight affects health } & 50 \% & 61 \% & 73 \% & .028 \\ \text { Understand need to lose weight } & 85 \% & 86 \% & 97 \% & .035 \\ \text { Want to lose weight } & 80 \% & 90 \% & 94 \% & .037 \\ \text { Previously attempted loss } & 80 \% & 87 \% & 94 \% & .05\end{array}$

CONCLUSION: The low literacy rates among overweight and obese patients at this public hospital are alarming. Patients with low literacy are significantly less likely to understand the adverse health consequences of obesity and the need to lose weight, and to report a desire weight loss. Health care providers should tailor both written and oral weight loss information to accommodate the needs of low literate patients.

PATIENT ILLITERACY: UNDERDIAGNOSED. J.R. Marks ${ }^{1}$, M.L. Plews-Ogan ${ }^{1}$, J.B. Schorling ${ }^{1}$; ${ }^{1}$ University of Virginia, Charlottesville, VA (Tracking ID \#76438)

BACKGROUND: The 1992 National Adult Literacy Survey found over $21 \%$ of adults had only "rudimentary" reading and writing skills, and fifty million had "marginal" skills. Despite these statistics, most patient education materials are written above an 8th grade level. A 1995 study demonstrated that $41.6 \%$ of subjects could not discern directions to take medication on an empty stomach. The pamphlet sent to patients of the University of Virginia Internal Medicine residents' clinic to report test results and medication instructions is at approximately a 10th grade level. We postulate that many residents' clinic patients are likely to have sub-optimal literacy skills that may impede their healthcare, and their physicians may not be aware of the problem. Another recent study suggests this scenario may be a widespread issue.

METHODS: A number of screening tools exist to assess literacy. The Rapid Estimate of Adult Literacy in Medicine (REALM) incorporates 66 commonly used medical as well as lay terms used in practice. Based on the patients' ability to read these words, their score correlates with an approximate school-grade reading level. One hundred patients selected randomly at UVa's Internal Medicine residents' clinic participated. Correspondingly the residents predicted their patients' approximate reading levels. Additional variables including gender, race, age, and pay scale (a measure of patient income) were examined as possible literacy predictors.

RESULTS: Eleven percent of subjects were functionally illiterate. The national average is closer to $4 \%$. Almost half read below a high school level. Although most studies show older age as predictor of low literacy, here the only significant predictor was male gender. Race, age, and pay scale were not predictive parameters. With respect to how well physicians knew their patients' literacy levels, the overall correlation was relatively good. Providers identified their patients who read at a high school level, but their ability to discern poorer readers was significantly less accurate.

CONCLUSION: REALM is a literacy screening tool felt to have satisfactory validity and reproducibility. However, it is not a test of comprehension and may, therefore, actually underestimate functional literacy. This study supports the notion that literacy rates among the residents' clinic patients are sub-optimal, and physicians are often unaware of patients' limited literacy skills. Certainly illiteracy rates and predictive factors will vary in different geographic regions and in various types of clinics. Finally, although this study does not directly examine the impact of patient illiteracy \& lack of its recognition, this topic is an important future direction of research. Recognizing the exact nature of the problems and errors arising from this scenario may ultimately improve patient compliance, safety, and overall outcomes.

INTERNET ACCESS AND EMPOWERMENT: A COMMUNITY-BASED HEALTH INITIATIVE. C. Masi $^{1}$, Y. Suarez-Balcazar ${ }^{2}$, M.Z. Cassey ${ }^{3}$, L. Kinney ${ }^{4}$, Z.H. Piotrowski ${ }^{5}$; ${ }^{1}$ University of Chicago, Chicago, IL; ${ }^{2}$ University of Illinois at Chicago, Chicago, IL; ${ }^{3}$ West Suburban Hospital College of Nursing, Oak Park, IL; ${ }^{4}$ United Way/Crusade of Mercy, Chicago, IL; ${ }^{5}$ West Suburban Hospital Medical Center, River Forest, IL (Tracking ID \#73705)

BACKGROUND: As a key principle in psychology and public health, empowerment reflects perceived control, competence, and goal internalization. Empowerment is also an important 
element of Healthy People 2010 strategies for increasing quality and years of life and eliminating health disparities. We used community-based participatory research techniques and information technology to assist residents of a low-income community gain access to health information via the Internet. Assessing the impact of Internet access on empowerment among the intervention and comparison groups was the primary goal of this study. METHODS: Twenty-five self-selected community residents were given in-home Internet access, training, and technical support over a two year period. These participants were encouraged to serve as health information resources or "citizen leaders" on their block. A homepage with links to community-specific and general health information was created as was a physician-staffed e-mail service for medical questions. Citizen leaders were interviewed at regular intervals regarding frequency of accessing the Internet and types of information obtained. In addition, citizen leaders and 35 randomly selected neighbors were interviewed regarding empowerment and attitudes toward technology at baseline and one year following the intervention.

RESULTS: Citizen leaders reported accessing the Internet an average of 3 times per week. Among those who benefited from Internet use, citizen leaders cited themselves most frequently (43\%), followed by family members (29\%), neighbors (15\%) and others $(13 \%)$. Reasons given for accessing the Internet included making purchases (17\%), networking (16\%), entertainment $(16 \%)$, health $(14 \%)$, community events $(13 \%)$, education $(10 \%)$, employment $(6 \%)$, safety $(4 \%)$, and religion (4\%). Citizen leaders were similar to comparison group members in terms of empowerment at baseline. After receiving Internet access and training, empowerment related to personal and family health decision-making improved significantly among citizen leaders but not the comparison group. Affinity for and appreciation of information technology also increased among citizen leaders but not the comparison group.

CONCLUSION: Using community-based participatory research methods, we found that Internet access to community-specific and general health information can lead to increased empowerment and appreciation of information technology. These benefits accrued among the intervention group but not among a random sample of their neighbors. Further studies are needed to determine whether empowerment derived from Internet access is associated with improved health outcomes.

DIFFERENCES BY RACE IN THE CONTROL OF DIABETES AMONG MEDICARE MANAGED CARE BENEFICIARIES. A. McBean ${ }^{1}$, Z. Huang ${ }^{1}$, B. Virnig ${ }^{1}$, N. Lurie $^{2}$, B.E. Dowd ${ }^{1}$, D. Musgrave ${ }^{3}$; ${ }^{1}$ University of Minnesota, Minneapolis, MN; ${ }^{2}$ RAND, Arlington, VA; ${ }^{3}$ Centers for Medicare and Medicaid Services, Baltimore, MD (Tracking ID \#76781)

BACKGROUND: Guidelines established by the Diabetes Quality Improvement Project (DQIP) for the care of persons with diabetes have been adopted by the National Committee on Quality Assurance (NCQA) as part of the Health Plan and Employeer Data and Information Set (HEDIS) measures used by NCQA to evaluate the performance of managed care plans. Previous work has demonstrated differences by race in the HEDIS measures for Hemoglobin A1c, LDL-cholesterol testing and eye examination. This study investigated possible differences by race in the control of HbA1c and LDL cholesterol in elderly Medicare beneficiaries with diabetes enrolled in managed care plans.

METHODS: Individual-level information provided by managed care plans to the Centers for Medicare and Medicaid Services (CMS) for 1999 on whether or not a person's HbA1c was $<9.5 \%$ or their LDL-C was $<130 \mathrm{mg} / \mathrm{dL}$ (These are the DQIP/HEDIS standards.) was combined with information on the person's race, other person-level and plan-level characteristics obtained from CMS. Bivarate and multivatiate analyses were conducted to identify differences by race adjusting for individual and plan-level characteristics.

RESULTS: The overall rate of HbA1c control was $67.3 \%$, and LDL-C control was $44.5 \%$. Age-gender adjusted rates of control were highest among Asians (HbA1c $<9.5 \%=75.3 \%$ and LDL-C $<130 \mathrm{mg} \% / \mathrm{dl}=52.4 \%)$; followed by Whites $(68.0 \%$ and $45.4 \%)$, Hispanics $(67.3 \%$ and $44.5 \%$ ); and Blacks (59.4\% and 33.3\%). Bivariate analysis indicated that, generally, higher income, being in a non-profit plan, being in a plan with a lower percentage of minority group members, not being in an IPA model plan, large plan size and being in a plan in the West US Census Region was associated with greater control. After controlling for these and other individual and plan-level variables in the multi-variate analysis, the differences between Asians and Whites in HbAlc and LDL-cholesterol controldisappeared. The other between-race differences, between Whites and Blacks for both measures and between Whites and Hispanics for LDL-cholesterol control, remained and were significant at the $\mathrm{p}<0.01$ level.

An increase in the number of different comprehensive diabetes control measures was significantly associated with a greater level of HbAlc and LDL-C control in all race groups. For example, for HbA1c control among Whites, those who had all three diabetes care measures had an $18 \%$ greater rate of HbA1c control than those who only had an HbA1c test. For Blacks the improvement was $53 \%$; for Asians, $28 \%$ and Hispanics, $30 \%$.

CONCLUSION: There is room for significant improvement in the control of HbA1c and LDL-C levels among all races. Among the four race groups studied, the greatest differences exist between Blacks and the two groups: Whites and Asians.

UNDERSTANDING RACIAL DISPARITIES IN HIV: THE COMBINED USE OF ADMINISTRATIVE DATA AND A CLINICAL COHORT. K.A. McGinnis ${ }^{1}$, M.J. Fine ${ }^{2}$ M. Skanderson ${ }^{1}$, R.K. Sharma ${ }^{3}$, J.H. Wagner ${ }^{1}$, M. Rodriguez-Barradas ${ }^{4}$, L. Rabeneck ${ }^{4}$, A.C. Justice ${ }^{2} ;{ }^{1}$ Center for Health Equity Research and Promotion, VA Healthcare System (CHERP), Pittsburgh, PA; ${ }^{2}$ CHERP; University of Pittsburgh Center for Research on Healthcare, School of Medicine, Division of General Internal Medicine, Pittsburgh, PA; ${ }^{3}$ University of Pittsburgh, Graduate School of Public Health, Pittsburgh, PA; ${ }^{4}$ VA Health Services Research and Development Center of Excellence; Department of Medicine, Baylor College of Medicine, Houston, TX (Tracking ID \#74305)

BACKGROUND: Disparities in health care and outcomes by race are of growing concern in the United States. Few studies of persons with HIV have sufficient power to compare survival between racial groups. In this study we examine differences in survival by race using administrative data for HIV positive veterans in care and then using in-depth clinical data from a cohort of HIV positive veterans to better understand the possible etiologies of racial disparities in survival.

METHODS: We identified 5,676 HIV positive veterans from national administrative data from the entire Veterans Affairs (VA) Healthcare System between June 1999 and September 2001. Cox proportional hazards regression was used to evaluate whether survival differed by race. We then used data from the Veterans Aging Cohort 3 Site Study (VACS 3) to compare baseline health status (CD4 count, provider-estimated prognosis, and number of comorbidities and HIV-related conditions), clinical management (highly active antiretroviral therapy, prophylaxis, and frequency of CD4 and VL laboratory tests) and adherence to HIV medication (according to patient report), by race, using Chi-square tests. VACS 3 enrolled 881 HIV positive veterans at three VA outpatient clinics from June 1999 to July 2000; data were collected from patient and provider surveys and electronic medical records.

RESULTS: According to national VA administrative data, African-American and Hispanic veterans have higher mortality rates than white veterans with $\mathrm{HIV}(\mathrm{HR}=1.40, \mathrm{CI}=1.19-1.67$ $\mathrm{HR}=1.42, \mathrm{CI}=1.08-1.88)$. VACS 3 data indicate that compared to white veterans, AfricanAmerican and Hispanic veterans had a poorer provider prognosis $(\mathrm{p}=.006)$, and greater burden of medical comorbidities $(\mathrm{p}<.001)$ and HIV related conditions $(\mathrm{p}=.003)$. Hispanic veterans were more likely to have a low CD4 count than African-American and white veterans ( $p=$ .003 ). We found no statistically significant differences in clinical management or in adherence to HIV medication.

CONCLUSION: HIV positive minority veterans in care experience poorer survival than do white veterans in care. This difference appears to be due to baseline differences in severity of illness as measured by provider assessment, burden of comorbidities and HIV related conditions, and CD4 count. There were no large disparities by race in processes of care or adherence to treatment.

IMPACT OF HEALTH LITERACY ON PATIENTS WITH HUMAN IMMUNODEFICIENY VIRUS INFECTION. E.L. McNeill ${ }^{1}$, C.A. Estrada ${ }^{1}$, T. Pinn ${ }^{1}$, A.L. Baro ${ }^{1}$, C. Collins ${ }^{1}$, J.C. Byrd ${ }^{1} ;{ }^{1}$ East Carolina University, Greenville, NC (Tracking ID \#76481)

BACKGROUND: Health literacy requires that individuals use reading and writing skills to comply with follow-up visits, understand instructions, and learn about disease processes. Patients with lower health literacy have lower CD4 counts, higher viral loads, and are more likely to be nonadherent with antiretroviral therapy. This study expands on previous work by assessing the impact of low literacy and the human immunodeficiency virus (HIV) in patients from predominately socioeconomically depressed rural areas.

PURPOSE: We assessed the magnitude of low literacy in patients with HIV infection in Eastern North Carolina and explored the relationship between literacy level and general knowledge of HIV infection.

METHODS: In a preliminary cross-sectional study of 55 patients with HIV infection, we measured functional health literacy using the Rapid Estimate of Adult Literacy in Medicine (REALM). Patients' knowledge of HIV infection was measured using the HIV KQ-18, a validated questionnaire. The questionnaire was verbally administered. We obtained selfreported grade level by patient interview.

RESULTS: Seventy-six percent of the patients were African American. The mean age was 37 years. Twenty percent were employed, and $87 \%$ had incomes less than $\$ 15,000$ per year. Although all but one patient reported achieving at least a seventh grade education, low literacy ( $<7$ th grade) was present in twenty-four percent $(13 / 54)$ on the REALM. Patients with higher health literacy levels had higher scores of HIV knowledge $(\mathrm{P}<0.0001)$.

CONCLUSION: Low literacy was prevalent in patients' with HIV infection in our study. Patients with lower health literacy had poorer understanding of their disease process.

$\begin{array}{lccc}\text { Grade } & \text { Self-Report } & \text { REALM } & \text { Mean (SD) HIV KQ-18 } \\ <3 \text { rd } & 0.0 \% & 5.5 \%(3) & 5.7(4.2) \\ \text { 4th-6th } & 1.8 \%(1) & 18.2 \%(10) & 11.5(4.4) \\ \text { 7th-8th } & 7.3 \%(4) & 34.5 \%(19) & 12.7(3.9) \\ \text { HS or more } & 90.9 \%(50) & 40.0 \%(22) & 15.2(2.2)\end{array}$

RACIAL DIFFERENCES IN DISCRIMINATION EXPERIENCES AND RESPONSES AMONG MINORITY SUBSTANCE USERS. T. Minior ${ }^{1}$, S. Galea ${ }^{2}$, J. Stuber ${ }^{2}$, J. Ahern' ${ }^{2}$; ${ }^{1}$ Montefiore Medical Center, Bronx, NY; ${ }^{2}$ New York Academy of Medicine, New York, NY (Tracking ID \#74796)

BACKGROUND: Discrimination is associated with both mental and physical health, and may be a particularly important determinant of health among marginalized groups. This study assessed differences in discrimination experiences and responses to discrimination between Black and Latino active substance users in New York City.

METHODS: 500 Black and 419 Latino active substance users were recruited through outreach workers, service agencies, and word of mouth. We collected data about different types of discrimination experienced (e.g. discrimination due to race, gender, substance use, etc.), the domains in which it occurs (e.g. at work, with police, etc.), and participants' responses to unfair treatment.

RESULTS: Discrimination due to drug use was the most commonly reported type of discrimination among both Blacks and Latinos. Black respondents were more likely than Latinos to report discrimination due to their drug use $(79 \%$ to $70 \%$ ), race ( $39 \%$ to $23 \%$ ), poverty ( $38 \%$ to $26 \%$ ), gender ( $18 \%$ to $9 \%$ ), and sexual orientation (38\% to $6 \%$ ). However, among those reporting discrimination due to drug use, Latinos experienced more rejection from family $(81 \%$ to $70 \%$ ), friends ( $73 \%$ to $60 \%$ ), police ( $86 \%$ to $79 \%$ ), employers ( $72 \%$ to $56 \%$ ), and medical care professionals ( $29 \%$ to $18 \%$ ). Black respondents were more likely to respond actively to discrimination, whereas Latino respondents were more likely to internalize experiences. CONCLUSION: There are substantial differences in discrimination experiences and responses to discrimination between Black and Latino substance users. These differences 
may help explain racial and ethnic differences in health among marginalized populations, and identify avenues for effective, targeted intervention.

DIABETES, CARDIOVASCULAR DISEASE, AND OTHER ASSOCIATED RISK FACTORS IN ASIAN INDIANS IN THE UNITED STATES: A POPULATION-BASED STUDY. S.A. Mohanty ${ }^{1}$, S. Woolhandler ${ }^{1}$, D.U. Himmelstein ${ }^{1}$, D.H. Bor ${ }^{1} ;{ }^{1}$ Cambridge Health Alliance and Harvard University, Cambridge, MA (Tracking ID \#74006)

BACKGROUND: Many studies, mostly from outside the U.S., have found high rates of diabetes, coronary heart disease (CHD) and hypertension among Asian Indians. However, no population-based study in the U.S. has examined diabetes and CHD prevalence or risk factors in this group.

METHODS: We compared rates of diabetes, CHD, hypertension, obesity, and other CHD risk factors of 555 adult Asian Indians to 87,846 adult non-Hispanic Whites by analyzing National Health Interview Survey (NHIS) for 1997, 1998, 1999 and 2000

RESULTS: Asian Indians were less obese than non-Hispanic whites $(6.2 \%$ vs. $19.2 \%, \mathrm{p}<$ 0.0001), had lower rates of tobacco use, and were less physically active. Multivariate analysis controlling for age, sex, and BMI showed that Asian Indians had a higher rate of borderline or overt diabetes (AOR, 2.69; 95\% CI, 1.72, 4.21). Multivariate analysis controlling for age, sex and tobacco use showed that Asian Indians had lower rates of CHD (AOR, 0.69; 95\% CI, 0.29, 1.65 ) and hypertension (AOR 0.62, 95\% CI 0.44, 0.86) than non-Hispanic Whites.

CONCLUSION: Asian Indians in the U.S. had higher rates of diabetes despite lower BMI. Unlike Asian Indians in India, the U.K., and Canada, Asian Indians in the U.S. have low rates of CHD and hypertension. Research and preventive efforts should focus on reducing risks for diabetes while identifying and preserving protective factors for CHD and hypertension in Asian Indians.

HEALTH CARE UTILIZATION OF IMMIGRANTS IN THE UNITED STATES: AN ANALYSIS USING THE MEDICAL EXPENDITURE PANEL SURVEY (MEPS) AND NATIONAL HEALTH INTERVIEW SURVEY (NHIS). S.A. Mohanty ${ }^{1}$, S. Woolhandler ${ }^{1}$ D.U. Himmelstein ${ }^{1}$, D.H. Bor ${ }^{1}$; ${ }^{1}$ Cambridge Health Alliance and Harvard University, Cambridge, MA (Tracking ID \#74019)

BACKGROUND: Recent media accounts have portrayed immigrants in the U.S. as high utilizers of health care who threaten to overburden tax-funded services. We analyzed the health care utilization patterns of immigrants in the U.S.

METHODS: We linked the 1998 Medical Expenditure Panel Survey (providing data on medical care utilization) and the 1996-1997 National Health Interview Survey (which includes information on immigration status) to determine the relationship between immigrant status and health care expenditures.

RESULTS: Immigrants had 34\% lower total health care expenditures ( $\mathrm{p}<0.0001), 25 \%$ lower office-based visit expenditures $(\mathrm{p}=<0.01)$, and $29 \%$ lower prescription drug expenditures $(\mathrm{p}<0.0001)$ than the U.S.-born, a pattern that persisted after adjusting for age, race, income, insurance status, and perceived health status. Emergency department (ED), inpatient, and hospital-based outpatient expenditures for immigrants were non-significantly lower than for the native-born. Immigrant children had $44 \%$ lower total health care expenditures ( $\mathrm{p}=$ $0.0011), 37 \%$ lower office-based visit expenditures $(p=0.0001)$, and $30 \%$ lower prescription expenditures $(\mathrm{p}<0.0001)$ than non-immigrant children. U.S.-born children with immigrant parents also had low expenditures.

CONCLUSION: Immigrants' utilization of health care is low. The strikingly low utilization rates of immigrant children raise serious concerns about the adequacy of their care.

VIOLENCE IN PRIMARY CARE: IDENTIFICATION AND TRAINING, DOES IT WORK? C. Morier-Genoud ${ }^{1}$, P. Bodenmann ${ }^{1}$, B. Favrat ${ }^{1}$, M. Vannotti ${ }^{1} ;{ }^{1}$ Medical Outpatient Clinic of Lausanne, Lausanne, Vaud (Tracking ID \#74295)

BACKGROUND: Primary care physicians underestimate domestic violence as well as other forms of violence (public or institutional) and therefore victims are at risk for further episodes of violence with psychological and physical consequences. The aim of the study was i) to assess the impact of an educational program for internal and family physician trainees to identify violence among swiss natives and foreigners, ii) to evaluate by a follow-up study the consequences of the interview for positive patients.

METHODS: In an academic primary care center, we conducted a pre/post-medical visit interview at baseline and following an educational program for doctors. All patients aged over 18 years who came to consult without appointment were included. The questionnaires were presented in the main foreign languages spoken by our patients. They addressed sociodemographics, SAFE questions for actual and past violence, patient'sown violence and if they were asked about violence during the current visit and what was said and proposed. The physicians - not informed about the aim of the study - were questioned on the principal medical problems brought up during the consultation and the recommended solutions. The educational program was a 3-hour workshop consisting in an interactive lecturer, case presentations, videotaped role plays and handouts summarizing the key points of the presentation. A post intervention survey took place 1 month later. All patients reporting domestic violence in the last year were followed-up and questioned again between 3 and 5 months after the first interview, all together with the doctors who took part in the study.

RESULTS: Prior to intervention, of 214 patients, 178 accepted to participate but 153 came to the post-visit interview. Mean age of patients included in the study was 35.9 (SD: 14), 55.1\% were women and $63.5 \%$ foreigners from 51 different nationalities. $12.4 \%(22 / 178)$ of the patients (16 men and 14 women) included in this study reported being victims of physical violence during the last year and $10.7 \%(19 / 178)$ of psychological violence (11 persons declaring both forms of violence). After the educational program, of 232 patients, 188 accepted to participate and 155 came for the post-visit interview. Mean age was 36.9 (SD: 10), $47.8 \%$ were women and $67.7 \%$ foreigners from 48 different nationalities. $7.4 \%(14 / 188)$ of the patients (13 men and 12 women) included in this study reported being victims of physical violence during the last year and $8 \%(15 / 188)$ of psychological violence (4 persons declaring both forms of violence). Physicians identified only $13 \%$ (4/30) of the patients victims of violence prior to the educational program, versus $16 \%(4 / 25)$ after the educational program $(\mathrm{p}=\mathrm{ns})$. Prior to the training, $6.5 \%(10 / 153)$ of the patients seen after the consultation said that their doctor had asked them about violence, versus $7.1 \%(11 / 155)$ of the patients after the educational program $(\mathrm{p}=\mathrm{ns})$. Of $29,22(75.9 \%)$ patients who reported being victims of domestic violence were found in the follow-up. The frequency of violence either diminished $(4 / 22 ; 18.2 \%)$ or ceased $(17 / 22 ; 77.3 \%)$.

CONCLUSION: Prevalence of violence in the last year is relatively high and is often neglected by doctors. However a low intensity educational program for doctors does not improve the identification of violence. Neverless, patients in a current violent relationship said that participating in the study helped and that violence decreased or ceased a few months later.

THE FREE CLINIC'S ROLE AS A STITCH IN THE SAFETY NET: A NATIONAL SURVEY. M. Nadkarni ${ }^{1}$, M.J. Kelley ${ }^{1}$, D. Marineau ${ }^{1}$, J.T. Philbrick ${ }^{1}$; ${ }^{1}$ University of Virginia, Charlottesville, VA (Tracking ID \#75511)

BACKGROUND: Free clinics have emerged as a prominent component of the health care safety net. They are private, non-profit corporations that provide physician care, ancillary medical services and prescription medications at little or no charge, delivered by volunteer licensed health care professionals. Despite their long history, little is published about the scope and value of services that free clinics provide across our nation.

METHODS: The executive directors of 213 of the 345 operating free clinics registered on the national free clinic web site responded to a mailed survey eliciting information on patient demographics and the scope and financial value of clinical services provided during 2000 . We estimated the value of physician visits at $\$ 75$ and prescriptions at $\$ 45$.

RESULTS: $58 \%$ of patients were reported to be female, $54 \%$ White, $21 \%$ African American, and $16 \%$ Hispanic. Eighty percent were between age 19 and 64 . Most clinics' target population were uninsured patients $(56 \%)$ and those considered indigent or low income $(26 \%)$. Responding clinics were $64 \%$ urban and $36 \%$ rural. Funding for clinics came $47 \%$ from private sources, $14 \%$ corporate, $10 \%$ government, and $25 \%$ other.

CONCLUSION: Free clinics vary widely in the amount and type of services provided. They effectively leverage donations and volunteerism to provide care to underserved populations. The majority of the $\$ 6.4$ billion financial value provided by the responding clinics was due to free medication dispensing. Despite the growth of free clinics as one strand in the safety net, less than $1 \%$ of the uninsured U.S. population received care at responding clinics. Despite their important contributions, it appears that free clinics can only be a minor stitch in the repair our fraying safety net.

Year 2000 Totals $(n=213)$

$\begin{array}{lcrrrc} & \text { Mean } & \text { Median } & \text { Minimum } & \text { Maximum } & \text { National Total } \\ \text { No. Patients } & 1,941 & 971 & 33 & 18,355 & 345,575 \\ \text { Patient Visits } & 5,506 & 2,834 & 98 & 83,259 & 1,145,273 \\ \text { Physician Visits } & \$ 413,000 & \$ 213,000 & \$ 7,350 & \$ 6,240,000 & \$ 86.0 \text { million } \\ \text { Prescription Value } & \$ 43.6 \text { million } & \$ 21.1 \text { million } & 0 & \$ 603 \text { million } & \$ 6.37 \text { billion } \\ \text { Annual Budget } & \$ 459,000 & \$ 215,000 & \$ 200 & \$ 12,000,000 & \$ 93.2 \text { million }\end{array}$

ARE THERE CONTINUING ETHNIC DISPARITIES IN SCREENING FOR HIGH CHOLESTEROL? S. Natarajan ${ }^{1}$, P.J. Nietert ${ }^{2} ;{ }^{1} \mathrm{New}$ York Harbor Healthcare System \& the New York University School of Medicine, New York, NY; ${ }^{2}$ Medical University of South Carolina, Charleston, SC (Tracking ID \#76227)

BACKGROUND: Though hypercholesterolemia is a major risk factor for cardiovascular disease and ethnic disparities in cholesterol screening have been noted, national studies have not evaluated if ethnic differences in screening for hypercholesterolemia are narrowing or widening.

METHODS: The 1987-2001 Behavioral Risk Factor Surveillance System, a series of yearly cross-sectional population-based surveys of US adults, was analyzed using SUDAAN to obtain population estimates. Appropriate screening was defined as individual self-report that it had been less than 5 years since the last cholesterol check by a health professional. Ethnicity was categorized as white, black, and Hispanic, and the other ethnic categories were excluded. Unadjusted, partially adjusted (age, gender and race* year interaction) and fully adjusted (age, gender, income, education, and race*year interaction) proportions reporting cholesterol screening were evaluated by logistic regression. Ethnic disparities over time were formally tested by the race*year interaction.

RESULTS: The sample increased from 50,081 in 1987 (population estimate 117 million) to 212,510 in 2001 (population estimate 215 million). In 1987, the unadjusted (and partially adjusted) proportions reporting cholesterol screening were $48.5 \%(55.2 \%)$ for whites, $41.4 \%$ $(50.4 \%)$ for blacks and $36.9 \%(50.6 \%)$ for Hispanics, indicating that blacks and Hispanics were less likely to be screened than whites $(\mathrm{p}<.0001)$. By 2001, the unadjusted (and partially adjusted) screened proportions were $76.5 \%(77.4 \%)$ in whites, $73.7 \%(76.9 \%)$ in blacks and $60.7 \%(65.9 \%)$ in Hispanics indicating persistent white-Hispanic disparity $(\mathrm{p}<.001)$ but no continuing white-black disparity in screening $(\mathrm{p}>.05)$. Further adjustment for education did not significantly affect the results. Additional adjustment for income (fully adjusted model) substantially affected the proportions screened both in 1987 (and in 2001), being $54.7 \%$ $(73 \%)$ in whites, $55 \%(77.2 \%)$ in blacks, and $42.5 \%(57.9 \%)$ in Hispanics. This model found similar screening among blacks and whites $(\mathrm{p}>.05)$ but an increasing disparity between whites and Hispanics $(\mathrm{p}<.001)$. The test for trend indicated that though the white-black screened proportions were converging $(\mathrm{p}<.005)$, the white-Hispanic proportions were diverging $(\mathrm{p}<.001)$. 
CONCLUSION: National data indicate a substantial narrowing of the cholesterol screening gap between whites and blacks. Of concern, the gap between whites and Hispanics seems to be widening, emphasizing the need to target Hispanics for screening. In both blacks and Hispanics, income is an important mediator of the disparity. Future studies should elucidate the mechanisms by which income mediates this disparity.

HEALTH CARE EXPERIENCES OF LIMITED-ENGLISH PROFICIENT CHINESE AND VIETNAMESE-AMERICAN PATIENTS. Q. Ngo-Metzger ${ }^{1}$, M. Massagli ${ }^{1}$, B. Clarridge ${ }^{2}$ J. Moorhead ${ }^{2}$, R.B. Davis ${ }^{1}$, R.S. Phillips ${ }^{1} ;{ }^{1}$ Harvard Medical School, Boston, MA; ${ }^{2}$ UMASS, Boston, MA (Tracking ID \#75951)

BACKGROUND: Asian Americans are one of the fastest growing ethnic groups in the United States; $75 \%$ are foreign-born and $70 \%$ speak primary languages other than English. We evaluated how Chinese and Vietnamese-Americans who have no or limited English-language proficiency experience medical care in the U.S.

METHODS: We conducted a mailed survey of 4,410 Chinese and Vietnamese-American patients from 11 community health centers in 8 cities across the U.S. We randomly selected patients 18 and older to receive a questionnaire approximately 1 month after an office visit. Patients had the option of answering the survey in English, Vietnamese, or Cantonese or Mandarin Chinese. The mailed survey included a \$5 incentive and respondents were entered into a prize-drawing. Patients also received a reminder phone call in their primary language. We used logistic regression to examine how patient characteristics and problem experiences predict ratings of care at the last visit (excellent, very good, and good vs. fair or poor). Patient characteristics included: ethnicity, age, sex, marital status, education, place of birth, time in the U.S., self-assessed English-proficiency, use of interpreter, number of visits in the last year, and self-reported health status. Problem experiences included problems with: access to care, information and education, providers' respect for patient preferences, providers' emotional support, and continuity of care.

RESULTS: We received 3,258 completed surveys for an overall response rate of $74 \%$. The mean age was 53 years and mean length of time living in the U.S. was 12 years; $63 \%$ were born in mainland China, $27 \%$ in Vietnam, $9 \%$ in another Asian country, and 1\% were U.S.-born. When patients were asked regarding the language that they are most comfortable speaking, $59 \%$ reported Cantonese, 16\% Mandarin, 21\% Vietnamese, and 2\% reported English; 89\% spoke English not well or not at all. Seventeen percent rated their overall care at the last visit fair or poor. Problems experienced by patients included: $16 \%$ did not get an appointment as soon as they wanted; $34 \%$ did not understand health information given by their providers; $25 \%$ had questions that they wanted to ask but did not; $30 \%$ reported lack of trust in their providers; and $13 \%$ were dissatisfied with how they received their test results after the visit. After adjustment for patient demographics and site of care, factors associated with low ratings of care included: not being able to get care quickly (AOR 2.60, 95\% CI 1.99-3.39); not understanding health information given by providers $(1.83,1.42-2.35)$; not being able to ask questions $(1.78$, $1.35-2.34)$; not having trust in the provider (4.99, 3.86-6.44); and not being satisfied with how test results were given $(2.03,1.50-2.74)$.

CONCLUSION: In a national survey of primary care patients, Asian Americans with limited English-proficiency reported high problem rates with various aspects of care, which were strongly related to overall ratings. Improving specific processes of care may improve the quality of care for these populations.

TRUST IN PHYSICIAN, FACILITY, AND SYSTEM: QUALITATIVE DIFFERENCES BETWEEN ETHNIC GROUPS. K.J. O'Malley ${ }^{1}$, P.M. Haidet ${ }^{2}$, B. Sharf ${ }^{3}$, T.C. Collins ${ }^{1}$, A. Tranh ${ }^{4}$, M.L. Johnson ${ }^{1}$, N.P. Wray ${ }^{1} ;{ }^{1}$ Baylor College of Medicine, Houston, TX; ${ }^{2}$ Houston VA Medical Center, Houston, TX; ${ }^{3}$ Texas A\&M, College Station, TX; ${ }^{4}$ UNC-Chapel Hill School of Public Health, Chapel Hill, NC (Tracking ID \#74612)

BACKGROUND: Patient decision-making preferences have been shown to contribute to ethnic disparities in health care. Researchers have posited that patient trust plays an important role in patients' decisions; however, no study has explicitly focused on qualitative differences in patient trust across ethnic groups, where trust is examined at multiple levels of the system. The objective of this large qualitative study was to thoroughly explore African American (AA), Mexican American (MA), Vietnamese American (VA) and White American (WA) patients' feelings of trust toward physicians, facilities, and the US health care system. We sought to identify common elements of trust across ethnic groups and those that differed qualitatively across ethnic groups.

METHODS: We recruited 82 patients stratified by ethnicity and type of system (city/charity, private, veterans' administration, alternative medicine) who reported interaction with multiple levels of the system over the past two years. Three trained interviewers conducted 18 in-depth focus groups designed to elicit patients' feelings of trust. Two primary and five secondary reviewers analyzed transcripts and identified initial themes. Reviewers compared findings, resolved discrepancies, and incorporated emergent themes into a hierarchy.

RESULTS: Regardless of ethnicity and level of system, three trust themes emerged. Trust related to humanistic and ethical behaviors (e.g., care, prioritizing patient care over all else, courtesy of staff, respect, honesty, confidentiality, discrimination, etc), technical competency (e.g., skill, knowledge, thoroughness, ability to explain procedures and tests, reputation, safeguards against mistakes, etc), and organization of care (e.g., promptness, availability of providers, atmosphere, access limitations, etc). We identified qualitative differences in patients' trust across ethnic groups. For a few examples, AA patients associated mistrust with health care providers who spoke disrespectfully to them, MA patients associated mistrust with lack of communication across healthcare providers and unnecessary treatments, and VA patients associated mistrust with unethical behaviors, such as prioritizing the making of money over patient care.

CONCLUSION: Patients share many feelings of trust or distrust in physicians, facilities, and the system; however, certain aspects of trust differed qualitatively across ethnic groups. Understanding aspects of patient trust and changing the system to improve these aspects will help eliminate ethnic disparities in health care.
ETHNIC DIFFERENCES IN CORONARY HEART DISEASE AND STROKE MORTALITY IN CALIFORNIA, 1990-2000. L. Palaniappan ${ }^{1}$, Y. Wang ${ }^{1}$, S.P. Fortmann ${ }^{1}$; ${ }^{1}$ Stanford University, Palo Alto, CA (Tracking ID \#75291)

BACKGROUND: Coronary heart disease and stroke are important worldwide health problems. The recent rapid increase in ethnic diversity in the U.S. calls for investigation into potential differences in death rates among U.S. ethnic populations.

METHODS: Coronary heart disease and stroke death rates were compared for six ethnic groups (non-Hispanic white, Hispanic, non-Hispanic black, Chinese, Japanese, and Asian Indian) by sex and age (25-44, 45-64, 65-84, and 25-84) using 1990 to 2000 California death data. Proportional mortality ratios (PMRs) for each sex and age group were calculated by dividing the proportion of deaths due to CHD or stroke in each ethnic group by the proportion of deaths due to CHD or stroke for the total population and are expressed as a percentage. RESULTS: Proportional mortality rates from coronary heart disease were highest in Asian Indian men and women (PMR 161 and 144 respectively). The greatest excess was observed in young Asian Indian men (PMR 299 in the 25-44 age group), and middle-aged Asian Indian women (PMR 167 in the 45-64 age group). Mortality rates from stroke were highest in Chinese men and women (PMR 160 and 140 respectively) when all ages were combined. Age stratified analysis revealed the greatest excess risk for stroke in young Asian Indian, Japanese, and Chinese men (PMRs 274, 228 and 200 respectively in the 25-44 age group). In women, the highest risk groups for stroke were Asian Indians in the 45-64 age group (PMR 181) and non-Hispanic blacks in the 25-44 age group (PMR 160).

CONCLUSION: Substantial ethnic differences exist in coronary heart disease and stroke mortality. These findings underline the importance of $\mathrm{CHD}$ as a cause of death for Asian Indians and stroke as a cause of death for Chinese. As ethnic diversity grows, further research into these differences is required to identify their causes and to treat populations at higher risk.

\section{Men Age 25-84}

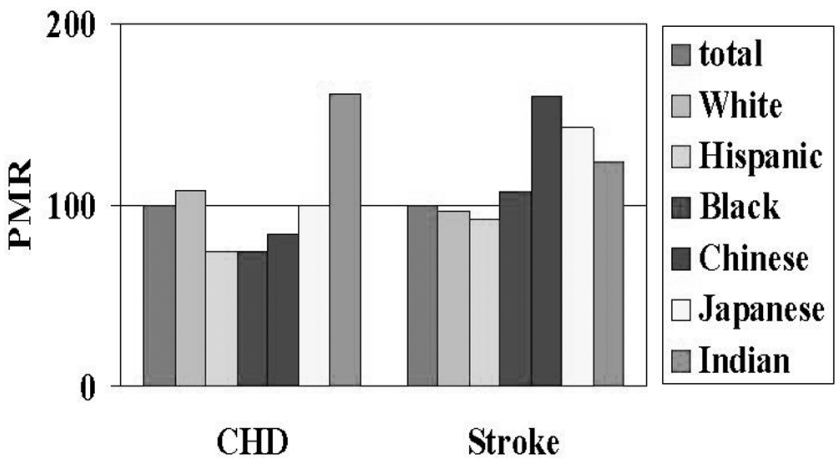

Proportionate Mortality Ratios for Coronary Heart Diasease and Stroke Mortality for Men 25-84 years

\section{Women Age 25-84}

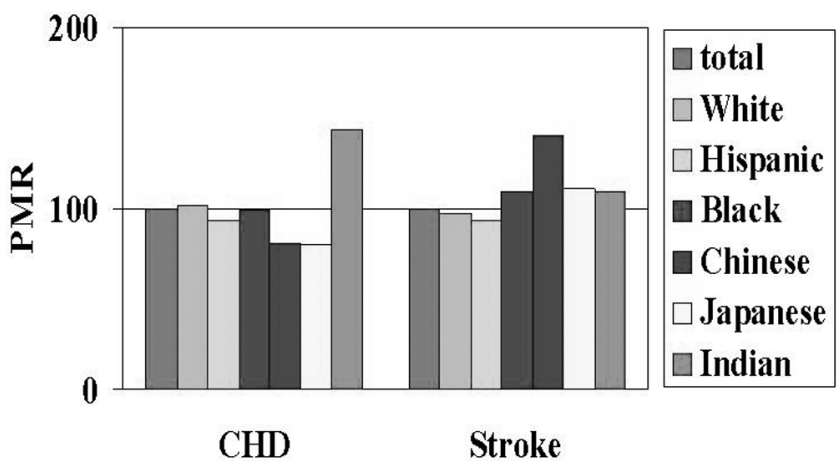

Proportionate Mortality Ratios for Coronary Heart Diasease and Stroke Mortality for Women $25-84$ years

SUBSTANCE ABUSE TREATMENT AND ADHERENCE TO ANTIRETROVIRAL THERAPY AMONG HIV-INFECTED PERSONS WITH ALCOHOL PROBLEMS. A. Palepu ${ }^{1}$, N.J. Horton ${ }^{2}$, N. Tibbetts ${ }^{3}$, S. Meli ${ }^{2}$, J.H. Samet ${ }^{2} ;{ }^{1}$ University of British Columbia, Vancouver, British Columbia; ${ }^{2}$ Boston University, Boston, MA; ${ }^{3}$ DM-Stat, Medford, MA (Tracking ID \#75145)

BACKGROUND: Among HIV-infected persons with a history of alcohol problems, little is known about the association of substance abuse services with antiretroviral therapy (ART) adherence. We examined these issues among participants in the HIV-Alcohol Longitudinal Cohort (HIV-ALC) study. 
METHODS: A standardized questionnaire was administered to $349 \mathrm{HIV}$-infected participants with a history of alcohol problems regarding demographics, substance use, use of substance abuse treatment services, and adherence to ART. These subjects were followed every 6 months for up to 7 occasions. We defined substance abuse treatment services as any of the following in the past 6 months: 4 weeks in a half-way house or residential facility; 4 visits to a substance abuse counselor or mental health professional; or participation in any methadone maintenance program. Our outcome variable was 3- and 30-day self-reported adherence, dichotomized at $100 \%$ and $95 \%$ adherent, respectively. Other variables included in the multivariable model were the effect of time, age, gender, depressive symptoms (CES-D score $>=16$ ), perceived social support, use of alcohol and drugs in the previous 30 days, and doses of antiretroviral medication per day. Multivariable logistic regression models using generalized estimating equations were constructed to examine the association of substance abuse treatment services on uptake of antiretroviral therapy at baseline and on adherence over time.

RESULTS: We found that substance abuse treatment services were not associated with 3- or 30-day adherence. The following variables were negatively associated with 3-day adherence: use of drugs or alcohol in the previous 30 days $(0.23 ; 0.21-0.26)$; higher number of doses of antiretroviral therapy per day $(0.88 ; 0.78-0.99)$; whereas social support from friends was positively associated with 3 -day adherence $(1.06 ; 1.01-1.12)$. In our 30 -day adherence model, we found that severe depressive symptoms $(0.56 ; 0.38-0.81)$ and use of drugs or alcohol in the previous 30 days $(0.28 ; 0.18-0.44)$ were negatively associated with adherence.

CONCLUSION: Engagement of substance abuse treatment services over time did not have a significant effect on adherence. The effect of substance abuse treatment services on adherence to antiretrovirals and HIV-1 treatment outcomes among HIV-infected persons with alcohol and drug problems may need to be addressed in a clinical trial.

ALCOHOL USE AND INCARCERATION ADVERSELY AFFECTS HIV-1 RNA SUPPRESSION AMONG INJECTION DRUG USERS STARTING ANTIRETROVIRAL THERAPY. A. Palepu ${ }^{1}$, M.W. Tyndall ${ }^{1}$, K. Li ${ }^{2}$, B. Yip ${ }^{2}$, M.V. O'Shaughnessy ${ }^{1}$, M.T. Schechter ${ }^{1}$, R. Hogg ${ }^{1}$, J. Montaner'; ' ${ }^{1}$ University of British Columbia, Vancouver, British Columbia; ${ }^{2} \mathrm{BC}$ Centre for Excellence in HIVIAIDS, Vancouver, British Columbia (Tracking ID \#75195)

BACKGROUND: Alcohol use is common among injection drug users as is incarceration given the nature of most users' lifestyle. We examined the association of self-reported alcohol use and prior incarceration at the time of initiating antiretroviral therapy on subsequent HIV-1 RNA suppression among HIV-infected drug users.

METHODS: We obtained the demographics, recent incarceration history, drug and alcohol use history from the Vancouver Injection Drug User Study which is an open cohort study of injection drug users. The questionnaire closest to the date of initiating antiretroviral therapy was linked to the HIV-1 treatment outcome data from the British Columbia HIV/AIDS Drug Treatment Program. There were 234 VIDUS participants who accessed antiretroviral therapy through the Drug Treatment Program from August 1, 1996 to July 31, 2001. We created a multiple logistic model to examine the association of drug and alcohol use with our primary outcome of achieving at least two consecutive HIV-1 RNA levels below 500 copies $/ \mathrm{mL}$ after the start of antiretroviral therapy. We adjusted for potential confounding factors by including in the model age (per year), jail, methadone maintenance therapy, baseline log10 HIV-1 RNA, baseline CD4 cell count, initial antiretroviral regimen (HAART versus not), months on therapy and adherence as measured by pharmacy refill compliance (per $10 \%$ increment).

RESULTS: In terms of illicit drug use, 196 (84\%) reported injecting heroin and/or cocaine and $82(35 \%)$ reported any alcohol use at the time of initiating antiretroviral therapy. HIV-1 RNA suppression was achieved by $133(57 \%)$ of the drug users. Multiple logistic regression revealed that in the 6 months prior to initiating antiretroviral therapy, alcohol use (adjusted odds ratio [AOR] 0.32; 95\% CI 0.13-0.81) and incarceration (AOR 0.22; 95\% CI 0.09-0.58) were independently associated with lower odds of HIV-1 RNA suppression. Factors positively associated with HIV-1 RNA suppression included: adherence (AOR 1.27; 95\% CI 1.06-1.51); lower baseline HIV-1 RNA (AOR 1.30; 95\% CI 1.01-1.66); highly active antiretroviral therapy (AOR 4.10; 95\% CI 1.56-10.6); months on therapy (AOR 1.1; 95\% CI 1.06-1.14). CONCLUSION: Among HIV-infected injection drug users who were on antiretroviral therapy, any alcohol use and incarceration in the 6 months prior to initiating antiretroviral therapy was negatively associated with achieving HIV-1 RNA suppression. The identification and treatment of alcohol problems should be supported in this setting. As well, increased outreach to HIV-infected drug users recently released from prison to ensure continuity of care need to be further developed.

RACIAL DIFFERENCES IN NARCOTIC USE FOR CHRONIC PAIN. M.E. Pasanen ${ }^{1}$ C. Faselis ${ }^{2}$, J.E. Kurz ${ }^{3}$; ${ }^{1}$ University of Vermont, Burlington, VT; ${ }^{2}$ George Washington School of Medicine, Washington, DC; ${ }^{3}$ University of North Carolina, Chapel Hill, NC (Tracking ID \#76807)

BACKGROUND: Chronic pain is a major worldwide health issue. Both patients and physicians are often frustrated in its management. We are interested in factors influencing physician treatment choices, specifically the role patient race and socioeconomic status plays in these decisions.

METHODS: The 4P Study is an ongoing multi-center, cross-sectional study designed to assess the attitudes and behaviors of physicians and their patients with chronic non-malignant pain. The patients are recruited from primary care sites at the time of routine follow-up and complete a 160 -item verbally administered survey. The physicians complete both a general survey of attitudes toward patients with chronic pain and a brief survey of specific patient encounters. The surveys include demographic information and current and past treatment choices. Race is categorized as white/black. We performed bivariate analyses looking for significant associations with use of narcotics and specialty care. We then performed logistic regression for narcotic use controlling for age, gender, insurance status, income, years of education, degree of pain and comorbities.

RESULTS: We report preliminary data based on the first 342 patients (155 white and 129 black), and 435 physicians surveyed. In bivariate analysis, we demonstrated that white patients were more likely to be taking narcotics than black patients $(\mathrm{p}=0.015)$. When we performed a $\mathrm{t}$ test, white patients had significantly lower mean pain scores (5.75 with CI 5.38-6.12) than black patients (6.51 with CI 6.11-6.90) on a validated $0-10$ pain scale $(\mathrm{p}=0.006)$. There were no differences in the use of specialists (orthopedist, rheumatologist, pain specialist, and physical therapy) between black and white patients. Our SES indicators were income and education, and these characteristics were not associated with narcotic or specialty use. When we preformed logistic regression, narcotic use for white patients remained significant compared to black patients (OR 2.37, p $=0.01$, CI 1.23-4.58). Overall, $42.6 \%$ of white patients and $28.7 \%$ of black patients reported taking narcotics. In the physician survey, only $44 \%$ agreed with the statement "There are no racial differences for pain seeking behavior." (34\% neutral and $22 \%$ disagreed).

CONCLUSION: When experiencing chronic pain, white patients are more likely to receive narcotics than black patients, even though they have lower average pain scores. Further efforts are needed to better understand this major treatment difference and its impact on quality of life and functional status. Physician attitudes on the racial differences in pain seeking behavior may be an important factor.

MEASURING CLINICAL DISPARITIES IN A DEVELOPED AND A DEVELOPING COUNTRY. J.W. Peabody ${ }^{1}$, F. Tozija ${ }^{2}$, J.A. Muñoz ${ }^{3}$, R.J. Nordyke ${ }^{4}$, J. Luck ${ }^{5} ;{ }^{1}$ SF VAMC, UCSF, San Francisco, CA; ${ }^{2}$ Project Preparation Unit, Ministry of Health, Skopje, Macedonia; ${ }^{3}$ Escuela Superior de Economla y Negocios, San Salvador, EI Salvador; ${ }^{4}$ Protocare Sciences, Santa Monica, CA; ${ }^{5}$ UCLA, School of Public Health, Los Angeles, CA (Tracking ID \#75860)

BACKGROUND: The most pronounced health disparities are found between developed and developing countries. We explored a paradigm shift of how these disparities could be addressed. While health disparities are most commonly explained by differences in income, access or education - factors which can only be changed gradually over time-we wondered if more immediate improvements might be found by improving the quality of care. To evaluate this approach, we directly measured the differences in the quality of clinical care in a developing versus a developed country using clinical vignettes. Vignettes have been previously validated (against the gold standards of actor patients) and are known to more accurately reflect actual clinical practice than the medical record. Vignettes are ideal for cross national comparisons because they inherently adjust for differences in health system organization and patient case mix. METHODS: Randomly selected eligible Macedonian and U.S. physicians ( $>97 \%$ participation rate) completed vignettes for eight common outpatient conditions. Responses were judged against a master criteria list and scored as percent correct.

RESULTS: The overall score for U.S physicians was $67 \%( \pm 11 \%)$ compared to $48 \%( \pm 11 \%)$ for Macedonian physicians ( $\mathrm{p}<.05)$. These differences persisted across the eight cases, each site within country and by case-complexity. Compared to US physicians, $3.5 \%$ of Macedonian physicians matched the median score and 14.7 matched or exceeded the 25 th percentile. However, the top $5 \%$ of Macedonian physicians in all sites approached or-in one caseexceeded the median score in the U.S. sites.

CONCLUSION: Direct case-mix-adjusted comparisons of the quality of care are possible in disparate populations using vignettes. While overall average performance was considerably lower in Macedonia, these findings suggest that the best providers in a poor country setting are able to perform as well as their colleagues in the developed world. Policy that promotes improvements in the quality of clinical practice-as opposed to structural inputs-could lead to improvements in health in a shorter time frame than many current initiatives.

PROCESS OF CARE AND OUTCOME AFTER ACUTE MYOCARDIAL INFARCTION FOR PATIENTS WITH MENTAL ILLNESS: ARE THERE DISPARITIES? L.A. Petersen ${ }^{1}$, S. Normand ${ }^{2}$, B. Druss ${ }^{3}$, R. Rosenheck ${ }^{3}$; ${ }^{1}$ Houston VAMC, Houston, TX; ${ }^{2}$ Harvard University, Boston, MA; ${ }^{3}$ Yale University, West Haven, CT (Tracking ID \#76389)

BACKGROUND: Patients with mental illness are a vulnerable group. To assess whether there are disparities in process of care and outcome after acute myocardial infarction (AMI) for patients with and without mental illness, we used primary clinical data from 81 Veterans Affairs (VA) hospitals.

METHODS: We studied 4,340 veterans discharged with clinically confirmed AMI. We defined mental illness as an admission to an inpatient psychiatric or substance abuse unit in the 365 days prior to the index admission for AMI; or a secondary psychiatric diagnosis on the index admission for AMI; or receipt of specialized outpatient psychiatric or substance abuse services during the 365 days prior to the index admission for AMI. 859 (19.8\%) of the sample met this definition. Data on comorbid conditions, severity of AMI, and angiography findings were collected using the Cooperative Cardiovascular Project data collection instrument. Use of guideline-based angiography and medications was compared. We assessed age-adjusted use of angiography, angioplasty, or bypass surgery both during the index hospitalization as well as in the 90 days after the index admission in either VA or under Medicare financing, and ageadjusted use of thrombolytic therapy, beta-blockers, angiotensin converting enzyme inhibitors, or aspirin at discharge; and risk-adjusted 30-day and 1-year mortality.

RESULTS: Patients with mental illness were marginally less likely than those without mental illness to undergo in-hospital angiography (age-adjusted RR 0.90 [95\% confidence interval: $0.83,0.98]$ ), but there was no significant difference in the age-adjusted RR of coronary artery bypass graft surgery in the 90 days after admission $(0.85[0.69,1.05])$, or in receipt of medications of known benefit. For example, ideal candidates with and without mental illness were equally likely to receive beta-blockers at the time of discharge (age-adjusted RR $0.92(0.82,1.02)$. The risk-adjusted OR for death in patients with mental illness vs. those without mental illness within 30 days was $1.00(0.75,1.32)$, and for death within 1 year was $1.25(1.00,1.53)$.

CONCLUSION: VA patients with mental illness were marginally less likely than those without mental illness to receive diagnostic angiography, and no less likely to receive revascularization or medications of known benefit after AMI. Mortality at 1 year may have been higher, although this finding did not reach statistical significance. These findings are consistent with other studies showing reduced health care disparities in the VA for other vulnerable 
groups, and suggest that an integrated healthcare system with few financial barriers to health care access may attenuate some health care disparities. Further work should address how health care organizational features might narrow disparities in health care for vulnerable groups.

\section{RACE/ETHNICITY, SOCIOECONOMIC STATUS AND FUNCTIONAL STATUS AFTER} RENAL TRANSPLANTATION. R. Press ${ }^{1}$, T.L. Nickolas ${ }^{1}$, J. Radhakrishnan ${ }^{1}$, R. Barr ${ }^{1}$; ${ }^{1}$ Columbia University, New York, NY (Tracking ID \#73843)

BACKGROUND: Patients with end stage renal disease have a better functional status and are more likely to be employed after renal transplantation compared to patients who receive chronic dialysis. Little is known, however, about potential racial and socioeconomic disparities in functional status after transplantation. We hypothesized that African American race/ ethnicity and lower socioeconomic status (SES) would be associated with a worse functional status after renal transplantation.

METHODS: We studied the cohort of adult patients who underwent renal transplantation in the United States in 1990. Clinical, sociodemographic and functional status data were extracted from the United Network for Organ Sharing (UNOS), a database which includes all patients who undergo transpantation in the United States. Worse functional status was defined as requiring some or total assistance with activities of daily living or death. SES of the patients was approximated by using the percent pelow poverty level for the individual's zip code of residence at the time of transplantation. The percent below poverty level was obtained from the 1990 United States Census and divided into quintiles. The rate of days with a worse functional status was compared, and longitudinal analysis with generalized estimating equations and robust regression were used for multivariate analyses.

RESULTS: In the United States in 1990, 9401 individuals underwent renal transplantation. Patients were excluded if SES information was not available from the Census( $n=241)$, if the zip code was not recorded $(n=4320)$, if the functional status was not recorded by UNOS $(n=$ $1035)$ and if they were less than 18 years old $(n=384)$. Bivariate analysis showed that the percent of days with a worse functional status was $32 \%$ for African Americans compared to $22 \%$ for whites (RR $1.58,95 \%$ CI $1.34-1.85, \mathrm{p}<.0001$ ). The percent of days with a worse functional status was $30 \%$ for the poorest quintile of poverty level compared to $20 \%$ for the richest quintile (RR 1.60, 95\% CI 1.30-1.96, p <.0001). After controlling for age, sex, diabetes, hypertension, cadaveric donor, HLA mismatch and graft failure, the rate ratio of worse functional status was $1.22(95 \%$ CI $1.02-1.46, p=.03)$ for African Americans compared to whites. In the full model including SES, African Americans' functional status was not significantly different from whites $(\mathrm{RR}=1.14,95 \% \mathrm{CI} .94-1.39, \mathrm{p}=.19$ ) while the poorest quintile of poverty level remained significantly predictive of worse functional status (RR 1.28, $95 \%$ CI $1.02-1.62, \mathrm{p}=.04)$

CONCLUSION: African Americans and those with low income have a worse functional status after renal transplantation. Socioeconomic status was independently predictive of a worse functional status whereas race/ethnicity was not. This study suggests that disparities in functional status after renal transplantation exist and that they are related mainly to differences in income.

VALIDITY OF USING SURNAMES TO DEFINE CHINESE ETHNICITY. H. Quan ${ }^{1}$, W.A. Ghali ${ }^{1}$, S. Dean ${ }^{2}$, C.M. Norris ${ }^{3}$, P.D. Faris ${ }^{2}$, P.D. Galbraith ${ }^{1}$, M.M. Graham ${ }^{3}$ M.L. Knudtson ${ }^{1}$; ${ }^{1}$ University of Calgary, Calgary, Alberta; ${ }^{2}$ Calgary Health Region, Calgary, Alberta; ${ }^{3}$ University of Alberta, Edmonton, Alberta (Tracking ID \#76007)

BACKGROUND: There is growing interest in the study of ethnic and racial differences in population health and health care delivery. However, many health care databases lack information on the ethnicity of individual patients. Surnames have been proposed as an alternative source of information for defining ethnicity since surnames are usually passed on from generation to generation. Surname methodologies are promising but their validity has not been well studied. This study was designed to assess the agreement between self-perceived Chinese ethnicity and the ethnicity assigned using a Chinese surname list developed by Tjam (Can J Public Health 2001;2:138-42). We also assessed the effect of slight modifications to the surname list to derive a more sensitive surname method for defining Chinese ethnicity.

METHODS: Information on self-reported ethnicity and surname was obtained from patients who underwent coronary angiography in the province of Alberta, Canada in 2002. Tjam's Chinese surname list including 226 possible surnames was employed to define Chinese ethnicity. Discrepant (misclassified) cases were examined and an iterative process of modifications was applied to the name list through the selective addition and/or removal of individual surnames.

RESULTS: Of the 3059 patients reporting information on ethnicity, $0.6 \%$ perceived and reported themselves to be Chinese. That proportion was lower than the $1.1 \%$ of patients defined to be Chinese by the surname list. When compared with self-reported Chinese ethnicity (reference standard ), the surname list had a sensitivity of $66.7 \%$, positive predictive value of $35.3 \%$, specificity of $99.3 \%$ and negative predictive value of $99.8 \%$. We revised the surname list by removing 6 surnames that were common in both Chinese and other ethnic groups (i.e. COOK, KING, LONG, LEE, LAW, MAY) and adding 4 Chinese surnames (i.e. YEE, SHAN, YUE and MAR). The revised surname list had $83.3 \%$ sensitivity, $83.3 \%$ positive predictive value, $99.9 \%$ specificity and $99.9 \%$ negative predictive value.

CONCLUSION: The validity of defining Chinese ethnicity using Tjam's surname list is moderate. The revised Chinese surname list has a substantial agreement with self-reported ethnicity. However, the new Chinese name list needs to be validated in a large external database.

VALIDITY OF USING NAMES TO DEFINE SOUTH ASIAN ETHNICITY. H. Quan ${ }^{1}$, W.A. Ghali ${ }^{1}$, S. Dean ${ }^{2}$, C.M. Norris ${ }^{3}$, P.D. Faris ${ }^{2}$, P.D. Galbraith ${ }^{1}$, M.M. Graham ${ }^{3}$, M.L. Knudtson ${ }^{1}$; ${ }^{1}$ University of Calgary, Calgary, Alberta; ${ }^{2}$ Calgary Health Region, Calgary, Alberta; ${ }^{3}$ University of Alberta, Edmonton, Alberta (Tracking ID \#76027)

BACKGROUND: Facing a lack of information on ethnicity in many health databases, some researchers have instead used patients' names as an alternative source of information for determining ethnicity. To facilitate the implementation of such name methodologies, a computer program named Nam Pehchan was developed by researchers in the United Kingdom to identify individuals of South Asian ethnicity based on a programmed name table. Many researchers have used this program since the 1980s and its validity has been partially assessed in Europe, but not elsewhere. In this study, we further assessed the validity of Nam Pehchan by comparing the ethnicity assigned by the Nam Pehchan computer program with self-reported ethnicity among cardiac care patients in Alberta, Canada.

METHODS: Information on self-reported ethnicity and first and surnames was obtained from patients who underwent coronary angiography in Alberta, Canada in 2002. We defined South Asian ethnicity using the Nam Pehchan program in two ways: 1) first matching on surname only and then 2) matching on either surname or first name.

RESULTS: Of the 3059 patients reporting information on ethnicity, $1.2 \%$ perceived and reported themselves to be of South Asian ethnicity. That proportion was lower than the 1.4\% of patients defined to be South Asian by matching on surname and $1.9 \%$ by matching on either surname or first name. See table for the agreement between self-reported South Asian ethnicity (reference standard) and the ethnicity defined using Nam Pehchan. $\left({ }^{*} \mathrm{PPV}\right.$ : positive predictive value, NPV: negative predictive value)

CONCLUSION: The Nam Pehchan computer program has only moderate validity for identifying South Asian ethnicity. The name table contained in the program is perhaps in need of modifications that, if made, might improve the sensitivity of this name list method for identifying South Asian ethnicity.

ligreement between self-reported and Nam Pehchan defined South Asian ethnicity
$\begin{array}{lcccc}\text { Match on } & \text { Sensitivity } & \text { Specificity } & \text { PPV }^{*} & \text { NPV }^{*} \\ \text { Surname } & 59.5 \% & 99.3 \% & 50.0 \% & 99.5 \% \\ \text { First or surname } & 70.3 \% & 98.9 \% & 44.1 \% & 99.6 \%\end{array}$

GEOGRAPHIC VARIATIONS IN RACIAL DIFFERENCES IN CARDIAC PROCEDURE USE AMONG ELDERLY PATIENTS AFTER ACUTE MYOCARDIAL INFARCTION. S.S. Rathore ${ }^{1}$, H.M. Krumholz ${ }^{1}$; ${ }^{1}$ Yale University School of Medicine, New Haven, CT (Tracking ID \#76239)

BACKGROUND: Racial differences in cardiac catheterization (CATH) and coronary revascularization (REVASC) use among patients hospitalized with acute myocardial infarction have been previously reported. Given regional variations in cardiac procedure use, it is unclear whether racial differences in treatment also vary by region.

METHODS: We used data from the Cooperative Cardiovascular Project, a cohort of Medicare beneficiaries hospitalized with myocardial infarction in the United States between $1 / 1994$ and 2/1996, to determine if racial differences in the use of CATH and REVASC varied by geographic region. Black or white patients age $\geq 65$ years with confirmed myocardial infarction ( $\mathrm{n}=138,938)$ were classified by US Census region (Northeast, Midwest, South, and West) based on the location of their treating hospital and evaluated for CATH or REVASC (percutaneous coronary intervention or coronary bypass surgery) within 60 days of admission. Racial differences in CATH and REVASC use were evaluated nationwide and by Census region. Multivariable logistic regression analyses were conducted using a patient race/region interaction term to test if racial differences in procedure use varied by region and were independent of patient, physician, and hospital characteristics.

RESULTS: Black patients had lower rates of procedure use than white patients nationwide (CATH $39.9 \%$ vs. $46.5 \%, \mathrm{P}<0.001$; REVASC $21.9 \%$ vs. $31.2 \%, \mathrm{P}<0.001$ ). Racial differences in CATH and REVASC use varied by Census region. There were no racial differences in CATH use in the Northeast (CATH $38.9 \%$ black vs. $40.5 \%$ white, $\mathrm{P}=0.24$ ). Racial differences in CATH use were observed in the Midwest ( $43.4 \%$ black vs. $38.9 \%$ white, $\mathrm{P}<0.001$ ), but largest in the South $(39.2 \%$ black vs. $48.5 \%$ white, $\mathrm{P}<0.001)$ and West $(38.3 \%$ black vs. $48.6 \%$ white, $\mathrm{P}$ $<0.001)$. Similarly, racial differences in REVASC were smallest in the Northeast $(22.1 \%$ black vs. $26.7 \%$ white, $\mathrm{P}<0.001)$, greater in the Midwest $(24.7 \%$ black vs. $33.5 \%$ white, $\mathrm{P}<0.001)$, and largest in the South $(20.7 \%$ black vs. $32.0 \%$ white, $\mathrm{P}<0.001)$ and West $(22.9 \%$ black vs. $33.7 \%$ white, $\mathrm{P}<0.001)$. Regional variations in racial differences in CATH and REVASC use persisted after multivariable adjustment ( $\mathrm{p}<0.001$ for race $^{*}$ Census region interaction).

CONCLUSION: Although elderly black patients hospitalized with myocardial infarction have lower cardiac procedure use rates than white patients overall, this pattern varies by region and is markedly smaller in the Northeast.

PATIENT TRUST AMONG AFRICAN-AMERICANS AND CAUCASIANS. $\underline{\mathrm{K} . \text { Ravenell }^{1}}$ K. Armstrong ${ }^{1}$; ${ }^{1}$ University of Pennsylvania, Philadelphia, PA (Tracking ID \#77074)

BACKGROUND: Variation in patient trust among racial groups may contribute to racial disparities in health care in the US. Because of geographic variation in health care systems and health care outcomes, we hypothesized that there would be differences in trust in physicians across communities and across race.

METHODS: We analyzed data from the 1998-1999 Community Tracking Study (CTS) Household Survey, a national survey of health care in 60 sites. As the populations of interest were African-Americans and Caucasians, we restricted to analysis to the 12 large metropolitan statistical areas to ensure adequate minority representation. As the item-to-total correlation for all items was less than 0.26 , we analyzed individual items rather than creating a trust scale. Physician trust items included "doctor may not refer when needed","doctor influenced by insurance rules," "doctor perform unnecessary tests" and "trust doctor to meet medical needs" and were scored on a 5-point Likert scale from strongly agree to strongly disagree. Bivariate analyses and multivariate logistic regressions were performed to examine the likelihood of patients answering that they strongly/somewhat agreed with the positive item and strongly/ somewhat disagreed with negative items. Sociodemographic characteristics included age $(<45$, $45-64,>65$ ), sex , general health status (poor/fair, good, and type of insurance (none, public, private). 
RESULTS: The sample included 27,376 patients: $46 \%$ were male, $14 \%$ were AfricanAmerican, $80 \%$ were less than 45 years old, $70 \%$ had private insurance, $55 \%$ had a high school education of less, and $66 \%$ said their general health was very good or excellent. After adjusting for other sociodemographic characteristics, African-American race was inversely associated with each measure of trust: including disagreeing with doctor may not refer $(\mathrm{OR}=0.67$, $\mathrm{p}<.004)$, doctor influenced by insurance rules $(\mathrm{OR}=0.50, \mathrm{p}<.0001)$, and doctor orders unnecessary tests $(\mathrm{OR}=0.59,<0.001)$ and agreeing with the statement trust doctor to meet medical needs $(O R=0.79, p=0.006)$. Each measure of trust differed by site (LR Test $<0.001)$. In addition, there was a significant interaction between race and site for all of the measures except belieff that doctor would meet medical needs (LR test p-values $<0.03$ ). Type of insurance was significantly associated with all four items.

CONCLUSION: Differences in patient trust among African-Americans and Caucasians may point to perceived and actual racial disparities in quality health care. Having insurance may increase doctor-patient interaction and thus improve trust. Further study is needed to examine the impact of geographic location, and the interaction with race on trust and health care outcomes.

\section{INFLUENCE OF PHYSICIAN RACE VS. PATIENT-PHYSICIAN INTERACTIONS ON THE} EXPERIENCE OF HEALTH CARE. S. Saha ${ }^{1}$, J.J. Arbalaez ${ }^{2}$, L. Cooper ${ }^{2} ;{ }^{1}$ Oregon Health \& Science University, Portland, OR; ${ }^{2}$ Johns Hopkins University, Baltimore, MD (Tracking ID \#76323)

BACKGROUND: Racial disparities in health care may be due in part to problems such as mistrust and bias arising in relationships between patients and physicians of different race. We sought to determine whether such problems are a function of race discordance between patient and physician per se, or of the quality of patient-physician interactions in race-discordant relationships.

METHODS: We analyzed data from the Commonwealth Fund's2001 Health Care Quality Survey, a telephone survey of adults in the continental U.S. (response rate $54 \%$ ). We included white, black, Hispanic, and Asian respondents with a regular physician of known race. We constructed a scale measuring the quality of patient-physician interactions, incorporating respondents' ratings of physicians on listening, explaining, involving patients in decision making, time spent, and treating patients with respect (Cronbach's alpha 0.79 ). We tested the independent associations of patient-physician race concordance and quality of interactions with patients' reports of discrimination in health care, trust in physicians, and global satisfaction with care.

RESULTS: Respondent totals and proportions with race-concordant physicians were as follows: white $2851 / 85 \%$, black $771 / 25 \%$, Hispanic $710 / 28 \%$, and Asian $430 / 45 \%$. Nonwhite respondents reported discrimination when seeking health care more often than whites (W:2\%, $\mathrm{B}: 17 \%, \mathrm{H}: 13 \%, \mathrm{~A}: 17 \% ; \mathrm{p}<.0001)$. Asians and Hispanics were less likely than others to give physicians the highest rating on all elements of interpersonal care (W:44\%, B:42\%, H:34\%, $\mathrm{A}: 19 \%, \mathrm{p}<.0001)$, or to express a great deal of trust in their physicians (W:75\%, B:73\%, $\mathrm{H}: 64 \%, \mathrm{~A}: 61 \% ; \mathrm{p}=.0001)$. Asians were less likely than others to be very satisfied with their care (W:68\%, B:65\%, H:66\%, A:48\%; p = .0003). Controlling for age, sex, income, education, insurance, health status, language, duration of patient-physician relationship and quality of patient-physician interactions, race concordance was associated with higher satisfaction and lower perceived discrimination only among whites. For all racial groups, better patientphysician interactions were significantly associated with less perceived discrimination, greater trust, and greater satisfaction. Among individual elements of patient-physician interactions, spending adequate time with patients was the most consistent predictor of satisfaction, while physicians perceived as respectful earned the most trust in all racial groups.

CONCLUSION: The quality of patient-physician interactions had a greater influence than physician race on patients' experience of health care, satisfaction, and trust in physicians. Enhancing physicians' interpersonal skills may be the most effective way to improve "cultural competence" in health care.

IMPACT OF BELIEFS ON COLORECTAL CANCER SCREENING IN LATINOS. R. Salazar ${ }^{1}$, J.M. Walsh ${ }^{1}$, G. Gildengorin ${ }^{1}$, E.J. Perez-Stable ${ }^{1} ;{ }^{1}$ University of California, San Francisco, San Francisco, CA (Tracking ID \#75456)

BACKGROUND: Colorectal cancer is the third most common cancer in the United States. Previous studies have shown that Latinos have lower rates of screening compared with whites. The impact of beliefs on colorectal cancer screening in this population is largely unexplored. The purpose of this study is to identify current beliefs regarding colorectal cancer screening and the subsequent effect in Latinos.

METHODS: A telephone survey was conducted on a population of 50-74 year old Latinos, non-Latino whites and Vietnamese living in San Jose, California. Data for the Latino and white respondents was used to explore current beliefs regarding colorectal cancer screening and its impact on practices. A multivariate analysis, using multiple imputations to account for missing data, was used to identify those beliefs that significantly impacted screening.

RESULTS: The original study included 775 individuals who completed a telephone survey for a response rate of $30 \% .226$ (29.2\%) Latinos responded, 310 (40\%) were non-Latino whites, and $239(30.8 \%)$ were Vietnamese. The average age for all respondents was 61 years; approximately $50 \%$ were male. In Latinos, $50 \%$ reported having fecal occult blood testing (FOBT), 38\% reported having a sigmoidoscopy (SIG), and 29\% reported having a colonoscopy (COL). In comparison to whites, Latinos were less likely to have had FOBT (OR $0.65 ; 95 \%$ CI $0.46-0.92$ ) or a SIG (OR $0.61 ; 95 \%$ CI $0.43-0.87$ ). Overall, Latinos were more likely to have found FOBT embarrassing than whites $(21 \%$ vs. $8 \% ; \mathrm{p}<0.05)$. Similarily, more Latinos found SIG or COL embarrasing compared with whites ( $25 \%$ vs. $17 \%$; $<<0.05)$. Finally, fewer Latinos would want to know if "something were wrong" compared to whites $(85 \%$ vs. $94 \%$; p < 0.05$)$. Latinos were more likely to be afraid of having a FOBT because of a potential diagnosis of cancer than whites $(11 \%$ vs. $3.5 \%, \mathrm{p}<0.05)$. Furthermore, a great percentage of Latinos were afraid of having a SIG or COL for the same reason (potential diagnosis of cancer) when compared to whites $(13 \%$ vs. $7 \%$; $<<0.05)$. Thinking that a SIG or COL is embarrasing was associated with a decreased likelihood of planning for a COL in Latinos (OR 0.35; 95\% CI $0.16-0.78$ ). Those Latinos who did not want to know if "something was wrong" were less likely to plan on a SIG within the next 5 years (OR 0.14: $95 \%$ CI $0.03,0.68$ ).

CONCLUSION: Latinos have lower rates of CRC screening than whites. Certain beliefs may negatively influence CRC screening practices and plans for future testing in the Latino population. Further research is needed to better understand and thus overcome such barriers.

ARE PICTURES WORTH A THOUSAND WORDS? COMMUNICATION REGARDING MEDICATIONS IN A PUBLIC HOSPITAL ANTICOAGULATION CLINIC. D. Schillinger ${ }^{1}$, E. Machtinger ${ }^{1}$, F. Wang ${ }^{1}$, K. Win ${ }^{1}$, L. Chen ${ }^{1}$, M. Rodriguez ${ }^{1}$, A.B. Bindman ${ }^{1} ;{ }^{1}$ University of California, San Francisco, SF, CA (Tracking ID \#73997)

BACKGROUND: Miscommunication between patients and providers regarding medications is common. Measuring rates of miscommunication, as well as the difference between verbal and visual modes of communication, can inform efforts to more effectively communicate with patients about medications.

METHODS: We performed a study among chronic warfarin users in a public hospital anticoagulation clinic to assess concordance between patients' and providers' reports of patients' warfarin regimen. Bilingual research assistants asked patients prior to clinic visits to (1) verbalize their weekly warfarin regimen and (2) identify their regimen from a digitized color warfarin pill menu. We obtained providers' reports of patients' regimen from chart review. We categorized patients as having regimen concordance if there were no patient-provider discrepencies in both the number of pills and total dosage for each of the prior 7 days. We measured differences in patient-provider concordance when the regimen was assessed verbally or visually. We then examined whether the differences between verbal and visual concordance varied with patients' functional health literacy level and language status, adjusting for age, race, cognitive function, and complexity of regimen. We measured functional health literacy with the s-TOFHLA, and cognitive function with the CASI, both validated instruments.

RESULTS: We enrolled 169 patients, mean age 61 years, $57 \%$ of whom spoke English, $27 \%$ Spanish, and $16 \%$ Cantonese. Overall, only $48 \%$ of patients achieved verbal concordance and $62 \%$ achieved visual concordance with providers regarding the weekly warfarin regimen $(\mathrm{p}<.01)$. While visual assessment was more likely to lead to regimen concordance than verbal assessment for all patient groups, it was particularly beneficial for patients with communication barriers. Shifting the assessment from verbal to visual modality was associated with greater odds of achieving concordance for Cantonese vs English speakers (AOR 2.4, p = .05) and for patients with inadequate vs. adequate functional health literacy (AOR 2.6, $\mathrm{p}=.02$ ).

CONCLUSION: Medication miscommunication in this sample was common, but occurred less frequently when patients identified their regimen with a visual aid. These results suggest that medication assessment and education may improve by using visual aids, and that this mode of communication may be especially beneficial for patients with communication barriers.

FUNCTIONAL HEALTH LITERACY AND THE QUALITY OF PHYSICIAN-PATIENT COMMUNICATION. D. Schillinger ${ }^{1}$, A.B. Bindman ${ }^{1}$, A. Stewart ${ }^{1}$, F. Wang ${ }^{1}$, J.D. Piette ${ }^{2}$ ${ }^{1}$ University of California, San Francisco, San Francisco, CA; ${ }^{2}$ University of Michigan, Ann Arbor, MI (Tracking ID \#74421)

BACKGROUND: While patients with poor functional health literacy (FHL) have difficulties reading and comprehending written medical instructions, it is not known whether these patients also experience problems with verbal communication with their primary care physicians.

METHODS: We performed in-person interviews with 408 English- and Spanish-speaking patients with type 2 diabetes cared for by 86 primary care physicians in 2 primary care clinics at a university-affiliated public hospital to examine whether patients with inadequate FHL report worse communication with their primary care physician than patients with adequate FHL. We assessed patients' FHL using the previously validated abbreviated Test of Functional Health Literacy in Adults, and assessed patients' experiences of communication with their physician using scales from the previously valiated Interpersonal Processes of Care in Diverse Populations instrument.

RESULTS: In bivariate analyses, patients with inadequate FHL, when compared to patients with adequate FHL, reported worse experiences with physician communication across 5 of 7 domains, including general clarity, explanation of condition, explanation of processes of care, empowerment, and decision-making, but not elicitation of patient problems or explanations of self-care. After adjusting for patients' sociodemographic characteristics, treatment regimen, length of time in their physician's care, years with diabetes, and accounting for the clustering of patients within physician, patients with inadequate FHL were more likely to experience worse communication across general clarity (adjusted odds ratio [AOR] 4.74, 95\% C.I. 1.25-18.01, $\mathrm{p}=.02$ ), explanation of condition (AOR 4.29, 1.13-16.32, $\mathrm{p}=.03$ ), and explanation of processes of care (AOR 2.17, 1.01-4.68, p = .05).

CONCLUSION: Inadequate functional health literacy appears to be a marker for more pervasive communication problems between patients and their health care providers, particularly in the technical, explanatory domains of clinician-patient oral communication. Given the prevalence of inadequate FHL and the importance of communication to successful chronic disease self-management, research is needed to uncover the reasons that these patients experience worse communication and identify strategies to improve communication.

HEALTH LITERACY AND PATIENT SATISFACTION. J.A. Shea ${ }^{1}$, C. Guerra $^{1}$, J. Weiner ${ }^{1}$, A. Aguirre ${ }^{1}$, M. Schaffer ${ }^{2}$, D.A. Asch ${ }^{3} ;{ }^{1}$ University of Pennsylvania, Philadelphia, PA; ${ }^{2}$ Health Partners, Philadelphia, PA; ${ }^{3}$ Center for Health Equity Research and Promotion-PVAMC, Philadelphia, PA (Tracking ID \#76689)

BACKGROUND: Patient satisfaction is represents an efficient means to study processes of care and is itself an important outcome of care. However, most assessments of patient satisfaction depend on written, self-administered instruments, thereby potentially excluding the views of patients with lower literacy skills. The purposes of this project were to produce 
two alternatives to the traditional printed format, and learn how responses differ across formats and how they are related to literacy.

METHODS: Two alternatives were developed for the Consumer Assessment of Health Plans Survey (CAHPS), a 62 -item instrument that assesses satisfaction with 5 specific and 2 overal domains of health care: an automated telephone delivered format and one in which illustrations support key concepts. Prototypes were tested in samples of patients awaiting primary care visits in urban community based practices with large Medicaid and Hispanic enrollments. Patients completed (in English or Spanish) the short Test of Functional Health Literacy in Adults and a CAHPS instrument in one of the 3 formats. Analyses utilized ANOVA and ANCOVA to compare means among forms and demographic groups and stepwise regressions to look at the relative importance of format, literacy and demographics (language, sex, age, education, and ethnicity).

RESULTS: 2494 patients took one of the three instruments; $78 \%$ were female, $50 \%$ were younger than $40,51 \%$ had less than a high school education, and $62 \%$ were Hispanic. Overall, $25 \%$ had inadequate health literacy, $11 \%$ had marginal literacy and $65 \%$ had adequate literacy. For five subscales and one overall score, scores were consistently and significantly lower (less satisfied) for the automated telephone format (all ps <.01). In bivariate comparisons, literacy level was significantly related to all subscale and overall satifaction scores (all ps $<.05$ ). Language, sex, age, education, and ethnicity had less consistent relationships. Literacy remained a significant predictor of all satisfaction scores in stepwise regressions, controlling for type of instrument and other demographics. Patients at the lowest literacy level had more missing/illogical data (mean of 5.1 of a required 24 items compared to 1.5 for the highest literacy group) and took about 2 minutes longer, on average, to complete the instrument.

CONCLUSION: Health literacy is an important but often unmeasured consideration in assessing and interpreting patient satisfaction. However, differences in administration format that might be implemented to overcome limitations in literacy are associated with differences in measured satisfaction. More importantly, it is also clear that lower literate patients are less satisfied with their health plan. Reasons for these observed differences, and the impact upon health seeking behaviors and health outcomes needs to be explored.

PATIENT SATISFACTION AMONG HISPANICS UTILIZING AN URBAN EMERGENCY DEPARTMENT. J.E. Sidle ${ }^{1}$, C.J. Morales ${ }^{1}$, J.J. Linder ${ }^{1} ;{ }^{1}$ Indiana University Purdue University Indianapolis, Indianapolis, IN (Tracking ID \#77070)

BACKGROUND: Hispanics or Latinos constitute a rapidly growing, yet under-served, segment of the United States population. While many studies over the past decade have examined disparities in access to health care for Latinos, few have focused on patient satisfaction with health care services. This study examines patient satisfaction among Hispanics utilizing an urban emergency department. Secondary aims include the identification of demographic characteristics that may serve as predictors of patient dissatisfaction, measurement of differences in satisfaction between patients using different types of language assistance, and measurement of differences in satisfaction between different subsets of the Latino population.

METHODS: This study is a cross-sectional survey of patient satisfaction of Hispanic patients utilizing the Wishard Memorial Hospital Emergency Department (ED) in Indianapolis, Indiana. Patients who identify themselves as Hispanic at the time of initial triage into the ED are eligible for participation. Consent is obtained at the time of the ED visit. Patients are later contacted by telephone for completion of a 90 -item questionnaire with the assistance of a bilingual interviewer. The survey uses a modified version of an instrument developed by Harris and Swindle et al. from the Medical Outcomes Study Visit-Specific Questionnaire (MOSVSQ) and the American Board of Internal Medicine Patient Satisfaction Questionnaire. Mailed surveys are sent to patients who do not have phones or who cannot be contacted by phone after multiple attempts. Univariate analysis will be used to identify factors associated with lower satisfaction with care. These may include gender, age, educational level, language of survey, length of ER visit, type of language assistance, etc. Variables which show significance will be evaluated in a multiple regression model. In addition, differences in satisfaction between Hispanic ethnic subgroups will be evaluated in the same manner.

RESULTS: At present, 577 patients have consented for participation in the study, and 335 interviews have been completed. Data from the surveys is presently under analysis and will be completed in January 2003. Final results will be prepared for publication and will be shared with the ED administration and the appropriate hospital planning committees.

CONCLUSION: Results from this patient satisfaction study will identify patient attitudes and needs useful for improving care for Hispanics in the ED. In addition, these results will provide pilot data of Hispanic patient satisfaction that can serve as an important starting point for future interventional studies within this under-served patient population.

PAP SMEAR AND MAMMOGRAPHY COMPLETION RATES AMONG UNINSURED WOMEN UTILIZING A FREE ACUTE CARE CLINIC. R. Stroebel ${ }^{1}$, P. Targonski ${ }^{1}$, E. Tung ${ }^{1}$, S. Freytag ${ }^{2}$, R. Staver ${ }^{1} ;{ }^{1}$ Mayo Clinic, Rochester, MN; ${ }^{2}$ Salvation Army, Rochester, MN (Tracking ID \#76962)

BACKGROUND: A challenge in delivering preventive services to the uninsured is contacting high concentrations of patients. Free medical clinics may provide a unique opportunity to reach a highly concentrated population lacking adequate preventive services.

METHODS: Demographic data on 1133 consecutive patients seen at the Salvation Army Free Clinic (SAFC) in Rochester, MN from 1999-2001 was obtained through a self-completed registration form. Variables including sex, ethnicity, age, education, income and insurance status were collected. Age and gender appropriate preventive service rates were assessed. Multivariable logistic regression was used to identify factors associated with non-completion of breast cancer and cervical cancer screening in eligible women.

RESULTS: Among women 50 years or older, 13 of $81(16 \%)$ received mammography within two years preceding their SAFC visit. Among eligible women, 45 of 398 (11\%) reported having a pap smear completed within three years of their visit. None of the assessed demographic variables were predictive for non-completion of mammography in this population. Non-white race and less-than high school education were significant risk factors for non-completion of pap smear, but only explained $29.6 \%$ of the variance for probability of receiving a pap smear in multivariable logistic regression models.

CONCLUSION: The CDC Behavioral Risk Factor Surveillance System observed rates among long-term uninsured for mammography and pap smear at $80 \%$ and $68 \%$ respectively. The lower rates in the SAFC population represent a sub-epidemic of inadequate screening for breast and cervical cancer. The lack of identifiable demographic risk factors and the apparent under use of free state sponsored screening programs suggests that additional barriers are impacting this specific population. Possible factors include denial of risk, childcare concerns, prioritization issues, or system barriers within existing delivery systems. The location, structure, hours, and care delivery system of free clinics are designed to reduce such barriers. Our data suggests the SAFC does serve as a health care destination for an acutely underserved population. This concentrated population of underserved patients represents an opportunity for focused interventions to close this gap in preventive service delivery.

CERVICAL CANCER SCREENING ADHERENCE AMONG VIETNAMESE AMERICAN WOMEN. V. Taylor ${ }^{1}$, Y. Yasui ${ }^{1}$, N. Burke ${ }^{2}$, T. Nguyen ${ }^{2}$, E. Acorda ${ }^{1}$, H. Thai ${ }^{3}$, J.C. Jackson ${ }^{3}$; ${ }^{1}$ Fred Hutchinson Cancer Research Center, Seattle, WA; ${ }^{2}$ University of California, San Francisco, San Francisco, CA; ${ }^{3}$ University of Washington, Seattle, WA (Tracking ID \#76606)

BACKGROUND: Cancer registry data indicate invasive cervical cancer incidence rates are five times higher among Vietnamese than non-Latina White women in the United States. The objective of this study was to examine factors associated with cervical cancer screening adherence among Vietnamese American women.

METHODS: We conducted a population-based survey of randomly selected Vietnamese women during 2002. All participants were Seattle residents aged 18-64 years. The survey content was guided by the Adherence Model and an earlier qualitative study. In-person interviews were conducted by bilingual, bicultural survey workers. The primary outcome was Pap testing in the last two years.

RESULTS: The response rate was $82 \%$, and the survey was completed by 370 women. Sixtytwo percent of the respondents reported cervical cancer screening within the last two years. In bivariate comparisons, the following factors were associated with recent screening $(p<0.05)$ : marital status; having health insurance; having a regular source of care and regular provider; believing getting regular Pap tests decreases the risk of cervical cancer; knowing that Pap testing is necessary if asymptomatic, sexually inactive, and after menopause; reporting concern about pain and cancer being discovered were barriers to Pap testing; family member(s) and friend(s) had suggested Pap testing; doctor(s) had recommended Pap testing and had asked doctor(s) for Pap testing. The following variables entered in a stepwise logistic regression analysis: having a regular source of care $(\mathrm{OR}=2.3 ; 95 \% \mathrm{CI}=1.01-5.4)$, knowing Pap testing is necessary if asymptomatic $(\mathrm{OR}=3.8 ; 95 \% \mathrm{CI}=1.7-8.7)$, reporting concern about pain was a barrier to Pap testing $(\mathrm{OR}=0.5 ; 95 \% \mathrm{CI}=0.3-0.8)$, doctor(s) had recommended Pap testing $(\mathrm{OR}=3.8 ; 95 \% \mathrm{CI}=2.2-6.7)$, and had asked doctor(s) for Pap testing $(\mathrm{OR}=3.9 ; 95 \% \mathrm{CI}=$ $2.2-6.8)$.

CONCLUSION: This study confirms low levels of cervical cancer screening among Vietnamese Americans. Our results reinforce the important role of primary care physicians in improving Pap testing rates in Southeast Asian immigrant communities. Educational programs should include messages about the importance of Pap testing for all women.

INFANT AND PREMATURE MORTALITY DIFERENCES IN ARGENTINA: THE EXPLANATORY POWER OF SOCIOECONOMIC AND AVAILABILITY OF MEDICAL CARE DISPARITIES. $\underline{\text { S. Terrasa }}{ }^{1}$, F. Rubinstein ${ }^{2}$, N. Giraudo ${ }^{2}$, M. Bortman ${ }^{3} ;{ }^{1}$ Hospital Italiano, Argentina, Buenos Aires, Argentina; ${ }^{2}$ Hospital Italiano, Buenos Aires, Argentina; ${ }^{3}$ World Bank, Washington, DC (Tracking ID \#74453)

BACKGROUND: Argentine has important inter-districts disparities in structural poverty, availability of medical care and health outcomes (e.g. illiteracy rate: from 1 to $8 \%$; potable water availability: from 35 to $99 \%$; medical insurance: from 37 to $80 \%$; infant mortality rate: from $10 / 1000$ live births to $34 / 1000$ ). As social inequalities increased during the last 25 years, we tested the associations between them and health outcomes to help policy makers in their decisions and empower social actors in their demands.

OBJECTIVES: 1) To describe infant mortality rate (IMR), years of potential life lost excluding deaths during de first year of life (YPLL-1 to70), and premature mortality by tuberculosis (YPLL-TBC) by province; 2) Using the former as dependent variables, to analize what fraction of their inter-province variability in 1996 may be explained by differences in socioeconomic factors and availability of medical care in 1991 .

METHODS: Ecologic study based on two cross sectional analisys (1991 and 1996) of Argentina'sprovinces. Multiple linear regression analysis (first: variable selection, second: adjusting by potential confounders, third: model validation with 1999 dependent variables). Independent variables were grouped in seven domains: income, family structure, education; structural poverty and house conditions; medical availability; occupational information and urbanization level.

RESULTS: In unadjusted models, percent of houses with three or more persons living in the same room in 1991 explained $62 \%$ of the differences between provinces in IMR, 37\% of YPLL-1 to 70 and $60 \%$ of YPLL-TBC in 1996. Lack of health inssurance explained $34 \%$ of the differences in IMR, 20\% of YPLL-1 to 70 and 33\% of YPLL-TBC. Differences in degreee of education predicted $40 \%$ of the variation in IMR, 34\% of YPLL- 1 to 70 and $50 \%$ of YPLLTBC. Other good predictors were fertility, percent of people with an unqualified work and percent of houses without potable water (36\%, $52 \%$ and $38 \%$ of the differences in IMR). We couldn't find any association between physicians density and hospital beds with the evaluated outcomes. Multivariable model did not change the main results of the analysis. CONCLUSION: IMR and YPLL-TBC are strongly associated with structural poverty indicators and YPLL-1to70 is moderately so. The percent of houses with three or more persons living in the same room is a precise and easy to obtain indicator of this condition. IMR, YPLL-1to70 and YPLL-TBC are moderately associated with absence of health inssurance. 
Efforts must be made to dimish the deep disparities in Argentina that include half of our popualtion living in poverty and to improve accesibiliy to health care.

HOMELESSNESS IN A PUBLIC HOSPITAL: THE TIP OF THE HOUSING ICEBERG. M. Tsai ${ }^{1}$, R. Weintraub ${ }^{2}$, M. Kushel ${ }^{1}$; ${ }^{1}$ UCSF, San Francisco, CA; ${ }^{2}$ Stanford University School of Medicine, Palo Alto, CA (Tracking ID \#73850)

BACKGROUND: Although homelessness is associated with increased hospitalization rates and length of stay, the extent of homelessness among hospitalized patients has not been well characterized. We sought to determine the point prevalence of homelessness and housing instability among inpatients at an urban public hospital.

METHODS: We set out to interview all eligible patients at San Francisco General Hospital who were hospitalized on October 30,2002. We excluded patients $<18$, those in police custody or ICU, tourists, those who did not speak English, Spanish or Cantonese, and those deemed by their MD to be too ill to participate. The questionnaire included items on demographics, housing status and housing instability. We defined current homelessness as having spent at least one night on the street or in a homeless shelter in the past 30 days, or having no place to go other than street or shelter upon discharge. We assessed housing instability by using risk factors for future homelessness including history of prior homelessness, spending $>50 \%$ of income on housing, crowded housing, and feeling unsafe in their housing situation. We defined crowded housing as having greater than 2 persons per room.

RESULTS: 184 of 287 patients were eligible to be interviewed. 129 completed interviews (69\% of eligible); non-respondents did not differ from respondents by age, gender or ethnicity. $74 \%$ were interviewed in English, 15\% in Spanish and 11\% in Cantonese. $26 \%$ of all respondents reported current homelessness. Over half $(53 \%)$ of all respondents reported a lifetime history of homelessness, a third of whom $(30 \%)$ had their first episode before they were 18. Currently housed respondents had many risk factors for becoming homeless, including past history of homelessness $(33 \%)$, spending $>50 \%$ of total household income on rent $(53 \%)$, reporting crowded housing $(28 \%)$ and reporting feeling unsafe where they lived $(23 \%)$. CONCLUSION: The majority of inpatients at the public hospital we surveyed were either homeless or at high risk for future homelessness. Clinicians caring for safety net patients need to be aware of the fragile nature of their patients' housing when planning post-hospital care. Policymakers need to consider ways to help safety net institutions manage the disproportionate share of patients who are homeless or who have housing instability.

REGIONAL VARIATIONS IN DRUG TREATMENT AND EFFECT ON ER USE AND ANTIRETROVIRAL (ART) ADHERENCE OF HIV+ DRUG USERS. B.J. Turner ${ }^{1}$, C. Laine ${ }^{2}$ P. Yang ${ }^{1}$, W. Hauck ${ }^{2}$; ${ }^{1}$ University of Pennsylvania, Philadelphia, PA; ${ }^{2}$ Jefferson Medical College, Philadelphia, PA (Tracking ID \#76554)

BACKGROUND: New York State (NYS) drug treatment programs are concentrated in the New York City (NYC) region. We have reported that longitudinal drug treatment (LDT; $>6$ months in one program) was associated with markedly reduced hospitalization. We hypothesized that HIV+ drug users outside of NYC would have dysfunctional health care outcomes related to poor access to drug treatment.

METHODS: For persons enrolled in NYS Medicaid in 1996-97 and meeting tested screens for HIV and illicit drug use $(\mathrm{N}=11,556)$, study outcomes from 1997 Medicaid files were: repeated (2+) emergency room (ER) visits and, for persons on combination ART for $2+$ months ( $\mathrm{N}=5,095),>95 \%$ adherence based on a pharmacy measure. Using logistic regression, we adjusted first for demographics, residence (see table), clinical conditions from diagnoses on claims (ie, type of drug abuse, alcohol abuse, non-HIV chronic diseases, AIDS, mental health conditions) and hospitalization days in 1996. In the ER model, we also adjusted for 2+ ART for $>60$ days. LDT and usual source of medical care were entered last.

RESULTS: Of $11,556,25.0 \%$ had 2+ ER visits and, of 5,095 on ART, $22.1 \%$ were adherent. LDT was associated with less ER use (adjusted odds ratio $0.76,[0.69,0.84]$ ) and greater adherence $(1.29[1.11,1.48])$. As shown, LDT varied by region but most regional differences persisted after full adjustment.

CONCLUSION: These data bolster evidence of the benefits of drug treatment in HIV+ drug users. Yet, improved outcomes of drug users in NYC require further study as they may reflect models of care and social support of value for HIV+ drug users nationally.

NYS Regional Variations in Longitudinal Drug Treatment and Fully Adjusted Outcomes

NYC Longitudinal drug Adjusted odds of treatment $(\%) \quad 2+$ ER visits $(95 \% \mathrm{Cl}) \quad$ ART adherence $(95 \% \mathrm{Cl})$$$
42.0
$$

$$
1.00
$$

Other large city$$
\begin{aligned}
& 42.0 \\
& 46.6 \\
& 18.0
\end{aligned}
$$

1.00

$1.18[0.97,1.45]$

$1.35[1.10,1.65]$

$2.15[1.43,3.23]$

$1.72[1.19,2.48]$

\subsection{0}

$0.73[0.53,0.98]$

$0.59[0.41,0.82]$

$0.72[0.37,1.30]$
$0.44[0.17,0.95]$

Rural

18.8

HYPERTESNION AWARENESS IN PUERTO RICANS. C.M. Vergara ${ }^{1}$, A. Martin ${ }^{1}$,
F. Wang ${ }^{2}$, S. Horowitz ${ }^{1}$; ${ }^{1}$ Hartford Hospital, Hartford, CT; ${ }^{2}$ UCONN College of Pharmacy, Storrs, CT (Tracking ID \#74698)

BACKGROUND: There are few published reports in the literature describing the health status of Hispanic populations in the United States with chronic illness. Objective: This study aims to measure culturally-specific factors that affect disease awareness and compliance with healthy behaviors of Puerto Rican patients with hypertension.

METHODS: Patient Selection and Setting: Puerto Rican patients, with an established diagnosis of hypertension and have attended two or more visits with their respective primary

care provider over a one year period, were asked to participate in the study. No patients refused to participate in the study. Patients were selected from a hospital-based ambulatory center, which provides care to underserved populations of the surrounding community. Study Design: A single, bilingual interviewer administered a questionnaire in Spanish or English. Questions were divided into the following seven categories: patient knowledge and perception of the disease, diet and salt intake, tobacco use, alcohol use, weight control, exercise, and medication and alternative therapy use. Within each category were various questions designed to study patient's knowledge of hypertension, compliance with behavior modifications, and patient education by physicians. Statistical Analysis: Data from 19 con-secutive Puerto Rican patients was collected and chi-square tests and post-hoc power analysis were performed. Responses to the various questions were analyzed for correlation to patient demographics and socioeconomic variables, such as marital status, level of education, medical insurance status, etc. RESULTS: Use of herbal teas correlated significantly with each patient's primary language. More specifically, twenty-one percent of the patients who spoke Spanish only used herbal teas for treatment of their hypertension. Additionally, all patients who reported herbal medication or tea use had a middle school education. There were many trends towards statistical significance in the following categories: patient perceptions about hypertension risks and level of formal education; patient knowledge of hypertension and language spoken; salt intake and years of schooling; and correlation of herbal use with employment status, health insurance status and age.

CONCLUSION: Additional research must be undertaken with larger sample sizes to give validity to these trends. This study provides evidence of the special issues that need to be considered in the management of hypertension in a specific ethnic patient population. In particular, little is known about hypertension in Puerto Ricans esiding in the United States.

RACE-SPECIFIC MORTALITY EFFECTS OF MARKET REFORM IN NEW JERSEY. K.G. Volpp ${ }^{1}$, A. Epstein ${ }^{2}$, S.V. Williams ${ }^{2} ;{ }^{1}$ Philadelphia V.A. Medical Center, Philadelphia, PA; ${ }^{2}$ University of Pennsylvania, Philadelphia, PA (Tracking ID \#75877)

BACKGROUND: Previous work has demonstrated that the quality of care for uninsured AMI patients in New Jersey (NJ) worsened substantially relative to patients in both the control state New York (NY) and nationally following passage of the Health Care Reform Act of 1992 (HCRA), a law that encouraged price competition between hospitals and reduced subsidies for hospital care for the uninsured. Although there is cross-sectional evidence from a variety of settings that blacks receive worse quality of care, little is known about whether cost-cutting health policy reforms disproportionately affect quality of care for black patients.

METHODS: We used NJ and NY administrative discharge data from 1990-1996 to examine risk-adjusted mortality for 1,595,098 patients with any of the 7 following conditions: AMI, stroke, pulmonary embolism, hip fracture, gastrointestinal bleeding, congestive heart failure, and pneumonia. Using linear probability regression, we modeled mortality risk as a function of individual patient risk characteristics, baseline mortality risk in $\mathrm{NJ}$ and $\mathrm{NY}$, intertemporal trends common to the two states, insurance status, and race. After ascertaining that the time trends in the two states ere similar pre-reform, we measured the effect of the law as the divergence in mortality time trends in NJ and NY post-1993.

RESULTS: Among white patients, there was an increase in mortality in NJ (relative to NY) for uninsured patients of 1.5 percentage points $(\mathrm{p}=.021)$ but no significant relative increase among insured patients. There was an additional relative increase in mortality for black patients in NJ overall $(0.7$ percentage points, $\mathrm{p}=.006)$ that was manifest only among the insured. Among black NJ patients with the 5 conditions for which admission is less discretionary (AMI, stroke, pulmonary embolism, hip fracture, and gastrointestinal bleeding), there were additional relative increases in mortality of 3.8 percentage points among the uninsured $(\mathrm{p}=.076), 1.7$ percentage points among the $<65$ insured $(\mathrm{p}=0.028)$ and 0.9 percentage points among Medicare patients $(\mathrm{p}=.228)$. The overall relative increase among black patients with these 5 conditions was 1.5 percentage points $(\mathrm{p}=.005)$.

CONCLUSION: A policy reform that was designed to reduce costs appears to have affected black patients more adversely than white patients. Because these effects were more pronounced in conditions for which admission is less discretionary, we speculate that the differences may result from differences in care after admission rather than differences in admission practices.

BARRIERS TO COLON CANCER SCREENING IN ETHNIC MINORITY POPULATIONS. J.M. Walsh', C. Kaplan ${ }^{1}$, B. Nguyen ${ }^{2}$, S. McPhee ${ }^{1}$, E.J. Perez-Stable ${ }^{1} ;{ }^{1}$ University of California, San Francisco, San Francisco, CA; ${ }^{2}$ Northern California Cancer Center, San Francisco, CA (Tracking ID \#76411)

BACKGROUND: To identify current colorectal cancer (CRC) screening practices and barriers to screening in the Latino, non-Latino white and Vietnamese populations.

METHODS: We conducted a telephone survey of 50-74 year old Latino, non-Latino and Vietnamese individuals living in San Jose, California. Participants were recruited using listed samples. We asked about demographics, whether they had ever undergone CRC screening, whether they were up to date with CRC screening, intentions to be screened and perceived barriers and facilitators to CRC screening. Univariate and multivariate analyses were conducted, using multiple imputations to account for missing data.

RESULTS: 775 individuals (40\% white; $29.2 \%$ Latino and 30.8\% Vietnamese) completed the survey (response rate $30 \%$ ). The average age was 61 and approximately half of respondents were female. Less than half of respondents $(46 \%)$ had graduated from college, $46 \%$ were employed, and only $7 \%$ of respondents were uninsured. Overall $23 \%$ of respondents reported receipt of fecal occult blood test (FOBT) in the past year, 28\% reported sigmoidoscopy (SIG)in the past 5 years and $27 \%$ reported colonoscopy (COL) in the past 10 years. Screening rates were generally lower in Latinos and Vietnamese. Latinos were less likely to have ever had FOBT than whites (OR 0.65: 95\% C.I. 0.46, 0.92). Latinos and Vietnamese were less likely than whites to have ever undergone sigmoidoscopy (OR 0.61: $95 \%$ C.I. $0.43,0.87$ for Latinos and OR 0.33: 95\% C.I. 0.23, 0.47 for Vietnamese). Vietnamese were less likely than whites to have ever undergone colonoscopy (OR 0.56: 95\% C.I. 0.38, 0.82). There were also significant differences in attitudes and perceived barriers by ethnicity. For example, although $79 \%$ of 
whites and $74 \%$ of Latinos stated that they would find SIG or COL uncomfortable, only $22 \%$ of Vietnamese would find it so $(p<0.05)$. While $21 \%$ of Latinos would find performing an FOBT embarrassing, only $8 \%$ of whites and $3 \%$ of Vietnamese felt this way $(\mathrm{p}<0.05)$. In multivariate analyses, for all ethnic groups, the most important factor influencing plans to undergo CRC screening was MD recommendation. Vietnamese were more likely than whites to plan to have SIG in the next 5 years (OR 2.3: 95\% C.I. 1.16, 4.46), but ethnicity was not a predictor of planning to have FOBT or COL.

CONCLUSION: Rates of CRC screening are lower in ethnic minority populations than in whites. Differences in attitudes and perceived barriers suggest that culturally tailored interventions to increase CRC screening will be useful in these populations.

RACIAL DISPARITIES IN RENAL INSUFFICIENCY RELATED TO LONGITUDINAL CHANGES IN DIABETES CONTROL. M.G. Weiner ${ }^{1}$, A. Cohen ${ }^{1}$; ${ }^{1}$ University of Pennsylvania, Philadelphia, PA (Tracking ID \#76718)

BACKGROUND: Previous work has shown that Black diabetics experience greater declines in renal function (RF) than Caucasians having similar degrees of diabetes control. The current study will assess the racial disparities in change in RF for patients whose diabetes control is stable, improves or worsens over two years.

METHODS: We determined the average HBA1c level for each diabetic patient having blood analyzed at a hospital laboratory in 1999 and 2000 who also had Creatinine tested in 1999 and 2001. We formed 4 cohorts based on the longitudinal change in diabetes control across the two years (good-good, poor-good, good-poor, poor-poor [threshold HBA1c $=8.0$ ]). Since RF varies inversely to Creatinine, we examined the percent change, rather than the absolute change in Creatinine in each cohort. The average change in RF was determined for both Blacks and Caucasians falling into each of the cohorts.

RESULTS: A total of 3166 patients had HBA1c testing in 1999 and 2000 and also had Creatinine testing in 1999 and 2001. The table shows the percent decline in RF for Blacks and Caucasians having differing patterns of diabetes control. Among the patients with good control in both years, RF in Blacks had declined nearly twice that of Caucasians over two years ( $\mathrm{p}=$ 0.007). In patients with poor diabetes control in both years, Blacks and Caucasians had similar levels of decline in renal function $(\mathrm{p}=0.17)$. In both Caucasian and Black patients with alternating diabetes control over the years, the degree of decline in RF was intermediate between the best and worst controlled patients. However, the change in RF for Blacks was not significantly different across the 4 levels of diabetes control $(F=0.72, p=0.54)$ whereas the trend was significant in Caucasian individuals $(\mathrm{F}=3.03, \mathrm{p}=0.02)$

CONCLUSION: This study extends earlier findings of worsening renal function in Black diabetics compared with Caucasians. The impact of diabetes on RF is demonstrated in finding that Blacks and Caucasians with consistently poor diabetes control had similar, clinically important declines in renal function. However, improved diabetes control in Blacks did not slow the decline in RF to the same degree as with Caucasians.

$\%$ Decline in Renal Function by Race and Diabetes Control (n) good1999good2000 poor1999good2000 good1999poor2000

poor1999-

$\begin{array}{llrrr}\text { Causcasian } & 4.8(1337) & 7.1(167) & 5.5(149) & 9.9(262) \\ \text { Black } & 8.4(683) & 10.0(159) & 10.1(119) & 11.9(290)\end{array}$

THE INFLUENCE OF PERCEPTUAL BARRIERS ON HEALTH CARE USE BY MEN OF COLOR WITH CHRONIC DISEASES. E.E. Whitaker ${ }^{1}$, L.S. Sadowski ${ }^{1}$, A.F. Brown ${ }^{2}$, Y. Wang ${ }^{1}$, A. Evans ${ }^{1} ;{ }^{1}$ John H. Stroger Jr. Hospital of Cook County/Rush Medical College, Chicago, IL; ${ }^{2}$ University of California, Los Angeles, Los Angeles, CA (Tracking ID \#76713)

BACKGROUND: Men, especially men of color, have historically used the health care system less than women. We evaluated whether or not men of color's perceptions of previous treatment by the health care system influences health care use.

METHODS: We analyzed data from the 2001 Commonwealth Health Care Quality Survey. Telephone interviews were performed during 2001 on a random, national sample of adults 18 and older, with oversampling for minority populations. We used the subset of data for men who reported at least one chronic condition: hypertension, heart disease, diabetes, asthma, depression and restricted the analysis to $4 \mathrm{racial} / \mathrm{ethnic}$ groups: Asian, Black, Hispanic, and White. We had 4 main outcome variables: 1) Seeing a doctor, 2) Putting off needed care, 3) Not filling needed prescriptions, and 4) Use of the Emergency Department vs. other clinical sites. These 4 variables were assessed over the past 12 months. We developed a Perceptual Barriers Scale (PBS) that included information about respondents' perception of treatment by the health care system (e.g. poor treatment because of race, language or insurance status)(Cronbach's $\alpha=.68$ ). To assess the relationship between the PBS score and health care use, we constructed logistic regression models adjusting for clinical and sociodemographic characteristics and regular source of care.

RESULTS: The study sample was 844 males with $62.1 \%$ White, $14.7 \%$ Black, $15.5 \%$ Hispanic, and $7.7 \%$ Asian. The mean age was $50.3 \pm 17.3$ (range 18-92). The mean PBS score was higher for men of color than white men $(\mathrm{p}<.001)$ and predicted putting off needed care $\mathrm{OR}=1.1$ (95\%CI: 1.1-1.2) or not filling needed prescriptions in the last year OR $=1.1$ (95\% CI: 1.0-1.2). Among the four racial/ethnic groups, Asian men saw a doctor and put off filling needed prescriptions in the last year in unadjusted analyses only. After adjustment, Black race was a non-significant predictor of putting off needed care $(\mathrm{p}=.07)$. Surprisingly, men with 2 or more chronic conditions were more likely to put off needed care compared with men with a single chronic condition $\mathrm{OR}=2.0(95 \% \mathrm{CI}: 1.3-3.0)$.

CONCLUSION: Negative perceptions about previous treatment by the health care system were associated with putting off needed care and not filling needed prescriptions. Despite more frequent negative perceptions among men of color, there were no ethnic disparities in health care use among men with chronic disease. Therefore, it appears that men of color may be better at overcoming perceived barriers.

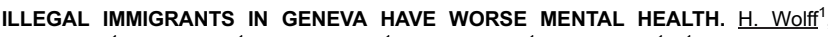
A. Morabia ${ }^{1}$, T. Collipal ${ }^{1}$, P. Hudelson ${ }^{1}$, C. Sanchez ${ }^{1}$, H. Stalder ${ }^{1}$; ${ }^{1}$ Department of Community Medicine, University Hospital of Geneva, Geneva, Geneva (Tracking ID \#75637)

BACKGROUND: Little is known about the health status of illegal immigrants who have major difficulties in access to health care. Their conditions of life are difficult: fear of the police, exploitement by their employers, separation from their families. We postulated that their health and specially their mental health status may be bad.

The aim of this study is to assess the socio-demographic characteristics and to evaluate the general health status and specifically the mental health status of uninsured, illegal immigrants in Geneva.

METHODS: All patients presenting at a free health care facility during 2 weeks in August 2001 and who did not have a legal residence permit were selected for the study. Those patients who agreed to participate in the study were administered a socio-demographic questionnaire, the COOP/WONCA-charts for general health care (range 1 (high) through 6) and the mental health inventory (MHI-5, range 1 through 100 (high)).

RESULTS: 185 patients presented to the health care facility, 26 (14\%) were excluded (mostly because they didn't understand neither French, Spanish or English) and $33(18 \%)$ refused to participate. The 126 patients enrolled in the study were mostly young (mean 34 years), female $(80 \%)$, of Latin-American origin $(86 \%)$ and had lived in Geneva for 2.4 years. $59 \%$ of study participants had children, but $72 \%$ had left at least one child in their home country. MHI-5 scores were low (50.9, CI 95\%: 47.6-54.2) compared to a mean score of 67.9 (CI 95\%: 66.8 $68.9)$ for a Swiss population. General health was found to be mediocre for "physical fitness" (2.6, SD 1.1), "feelings" (3.2, SD1.1)", "daily activities" (2.5, SD1.19), "social activities" (2.2, SD 1.2) and "overall health" (3.5, SD 0.9). Factors found to correlate with poor mental health included: living separated from the children, living alone or with more than 4 persons per room, and lack of a school diploma.

CONCLUSION: Targeted approaches are needed to respond to the specific problems of illegal immigrants. Medico-social structures that facilitate access to hard-to-reach populations is the key to assure adequate care and also to know more about the health status of illegal immigrants.

The general health and particularly the mental health of this mostly middle-aged Latin American population is poor, especially when separated from their children.

Health care professionals should pay particular attention to the psychological status of illegal immigrants.

WHICH DISEASES CONTRIBUTE MOST TO THE DISPARITY IN MORTALITY BETWEEN LATINO AND WHITE ADULTS? M.D. Wong ${ }^{1}$, T. Tagawa ${ }^{2}$, M.F. Shapiro ${ }^{1}$, W.J. Boscardin ${ }^{3}$, S.L. Ettner'; ' 1 University of California, Los Angeles, Los Angeles, CA; ${ }^{2}$ Chicago Medical School, North Chicago, IL; ${ }^{3}$ UCLA School of Public Health, Los Angeles, CA (Tracking ID \#76282)

BACKGROUND: Some evidence suggests Latinos have shorter life expectancy than whites, but which specific causes of death contribute most to this disparity is unknown.

METHODS: We estimated cause-specific mortality risks for Latino and white adults, age 25 and older, in the U.S. using 1986-1994 National Health Interview Survey (NHIS) data with linked death certificate data through 1997. Using these risk estimates, we created a statetransition Markov model to simulate mortality events for a cohort of adults followed from age 25 until death. From this model, we estimated the years of potential life lost (YPLL) due to specific causes of death for Latinos and whites, stratified by gender and adjusted for education. YPLL equals the difference between the maximum life expectancy, which we set at age 85 , and the actual age of death.

RESULTS: Among men, Latinos lost 13.3 YPLL per person (until age 85), compared with 8.9 years among whites - a difference of 4.3 years (95\% CI: $2.8-9.0$ ). Latinos lost 2.8 more YPLL than whites due to homicide. Thus, homicide accounted for $64 \%$ of the total YPLL disparity between Latino and white men. Other causes contributing to the YPLL disparity among men were stroke (12\%) prostate cancer $(8 \%)$, leukemia $(6 \%)$, and HIV $(6 \%)$. Death from ischemic heart disease was more common among White men than Latino men and, thus, accounted for $-9 \%$ of the total YPLL disparity. Among women, Latinos lost 9.2 YPLL per person, compared with 7.0 years among whites - a difference of 2.2 years (95\% CI: 1.6-4.6). The cause that contributed most to this disparity were accidents accounting for $23 \%$ of the total YPLL disparity. Accidents included motor vehicles accidents, which accounted for $9 \%$. Ischemic heart disease accounted for $13 \%$, followed by stroke (12\%), homicide (12\%), and leukemia $(11 \%)$. Hypertension, pneumonia and diabetes each contributed $8 \%$. Of note, these results were similar when estimating the YPLL before age 75 and before age 100 . CONCLUSION: All-cause mortality is higher among Latinos than whites, and the YPLL disparity between Latinos and whites is greater among men than among women. For men, homicide accounted for most of the YPLL disparity. For women, the top five contributors were accidents, ischemic heart disease, stroke, homicide and leukemia. These results have important public health and policy implications for future efforts to eliminate disparities in life expectancy between Latinos and whites.

ORAL CANCERS IN HIV-INFECTED PATIENTS: A POPULATION-BASED STUDY OF INDIVIDUALS IN CALIFORNIA. D. Zingmond ${ }^{1}$, W.E. Cunningham ${ }^{2}$; ${ }^{1}$ University of California, Los Angeles, Los Angeles, CA; ${ }^{2}$ University of California, Los Angeles, Santa Monica, CA (Tracking ID \#77051)

BACKGROUND: Oral cancers are recognized as important early manifestations of HIV/ AIDS. However, with the increasing survival of individuals with HIV-infection and the increasing prevalence of the disease among minorities, women, and older persons the epidemiology of HIV-related malignancies may be changing. 
METHODS: We studied all hospital discharges from the California Office of Statewide Health Planning and Development (OSHPD) annual Patient Discharge Database (PDD) for all patients ages 20 to 60 years old treated for acute conditions (except pregnancies) in nonFederal general acute care hospitals in California in 2000 in this observational case-control study. Primary cancers were grouped by ICD9-CM code with a focus on oral cancers. Kaposi's sarcoma and oral leukoplakia were excluded. HIV/AIDS status was identified by either DRG 488-490 or ICD9-CM code 042. Multivariate logistic models were developed to estimate the impact of HIV/AIDS and other patient characteristics on likelihood of oral cancers among all patients. Other independent variables included age, race, gender, and Charlson Comorbidity Index (modified to exclude AIDS or malignancies). Similar analyses were performed to examine the association of AIDS with lung cancer, which also has tobacco as a risk factor.

RESULTS: Overall, 1.23 million patients between the ages of 20 and 60 were admitted for acute care in California in 2000. Of these, 9,237 had a diagnosis of AIDS. Unadjusted comparisons showed a high rate of oral cancers in HIV/AIDS patients $(3.1 \%$ versus $0.21 \%$ of non-HIV/AIDS patients) and in Latino patients ( $0.61 \%$ versus $0.20 \%$ in non-Latinos). After multivariate adjustment, diagnosis of HIV/AIDS (OR $0.65,95 \%$ CI 17.6 to 23.1 compared to those without HIV/AIDS), male gender (OR $2.4295 \%$ CI: 2.23 to 2.63 vs. female), and Latino race (OR 3.05 , $95 \%$ CI: 2.74 to 3.39 vs. whites). Older patients had greater odds of an oral malignancy as compared to the reference group (age 30 to 34). Of note, a diagnosis of AIDS was not associated with greater odds of lung cancer.

CONCLUSION: Advanced oral cancers are more common among HIV/AIDS patients than matched non-HIV/AIDS patients and more common among Latinos than whites. Greater susceptibility to disease, differing health habits, and barriers to access may explain these findings in part. Primary care providers have a major role in the diagnosis and prevention of oral cancers in at-risk populations-HIV/AIDS and Latinos. 